\title{
The Noncommutative Geometry of Aperiodic Solids
}

\author{
Jean Bellissard ${ }^{1,2}$ \\ ${ }^{1}$ Georgia Institute of Technology, School of Mathematics, Atlanta GA 30332-0160 \\ ${ }^{2}$ Institut Universitaire de France
}

\section{Introduction}

These notes correspond to a series of Lectures delivered in July 2001 at the Summer School Geometry, Topology and Quantum Field Theory, that was organized at Villa de Leyva, in Colombia. Their purpose is to provide the students with a summary of a longstanding program with aim to describe aperiodic solids and their property with an appropriate mathematic framework. As explained in Section 2, the lack of periodicity in some solids does not allow to use the famous Bloch theory [5]. For this reason, physicists have developped various tools, such as finite scaling and the $\beta$-function of the renormalization group for disordered systems $[111,1]$, or such as the curved space representation for amorphous materials [72], or the cut-and-project method for quasicrystals. However, most of these technics are specific to sub-families of materials. The program presented here intends to give a general theory valid for all kinds of aperiodic solids. The framework provided by Noncommutative Geometry, as proposed by A. Connes [41] since the late seventies, will be shown to be the right tool replacing Bloch's theory whenever the translation invariance, that occurs in crystals, is broken. The main difference is that the so-called Brillouin zone becomes a noncommutative manifold, with a non trivial topology. In these notes, only the topological aspects and few of its consequences in physics are investigated. The systematic study of the transverse Geometry as well as the $N$-body problem [105] are not treated here and are left for future developments.

The main construction is the notion of Hull of an aperiodic solid. This is the content of Section 2 and Section 3. Several examples are proposed, impurities in a semiconductor, quasicrystals, tilings. It will be shown that the Hull is actually determined by the Gibbs thermodynamical ground state of the set of atoms. This Gibbs state also determines in a unique way various thermodynamical properties such as the diffraction pattern, the 
electronic density of states or the vibrational density of states (phonon modes). Once the Hull is constructed, it leads to the construction of the Noncommutative Brillouin zone (NCBZ) and its Geometry. Then the description of electrons in the one-particle approximation, or of the phonons in the harmonic approximation follows easily. No attempt to account for the large number of results obtained in the eighties and later concerning the spectral properties for both electrons and phonons will be made here. The reader is invited to look at $[43,33,86]$ concerning spectral results on disordered or quasiperiodic systems or at [18] concerning transport properties with anomalous spectra or diffusion. A special emphasis will be put upon recent results obtained to compute the $K$-theory of the NCBZ, especially in the context of the so-called gap labelling theorem (see Section 4). This theorem was formulated in the early eighties in its most general form [12] and has been given many illustrations in the case of one-dimensional systems during the eighties [16]. It required however another decade to get precise results for systems in higher dimensions. The present notes will conclude on a short description of what is still today the most spectacular application of the Noncommutative Geometry to realistic physics, namely the integer quantum Hall effect $[22,41]$.

Acknowledgments: The author wishes to express his warm thanks to the organizers of the Summer School, that was held in one of the most beautiful place in the world that is Villa de Leyva, for giving him the opportunity to deliver this series of lectures. He also wishes to thank the Colombian students of this school for their enthusiasm and their willingness to learn the best of modern Theoretical Physics. They all showed that in a country plagued by terrorism and corruption for decades, there is hope for individuals through these exceptional men and women who are working with competence and courage at creating a future of peace and progress. The author wishes also to thanks his collaborators past and present without whom this program could not have been developed.

\section{Mathematical Description of Aperiodic Solids}

In Solid State physics, most of the theory available in textbooks concerns periodic crystal in which the Bloch theory applies. Since the mid-sixties, however, physicists started wondering about what happens for non periodic materials. In this section we propose a formalism developped in various papers since the early eighties [19, 13, 16, 22, 23, 24], that is a substitute to Bloch theory, whenever Bloch theory fails to apply.

\subsection{Examples of Aperiodic Solids}

The structure of solids was investigated from the second half of the 19th century mainly from the point of view of their macroscopic properties. Crystals were then the focus of attention. It was not until the first experiment using X-rays by von Laüe in 1911 [64], that the microscopic structure of solids could be observed. For obvious reasons of symmetry, 
perfect crystals have been the focus of attention until quite recently. Besides, many of the known materials exhibit a microscopic structure that is perfectly periodic. This is the case for metals, such as copper, iron, aluminium, or for many ionic salts, like sodium-chloride, or most oxydes. It is one of the most challenging questions, even nowadays, to understand why perfect crystals are so common in nature.

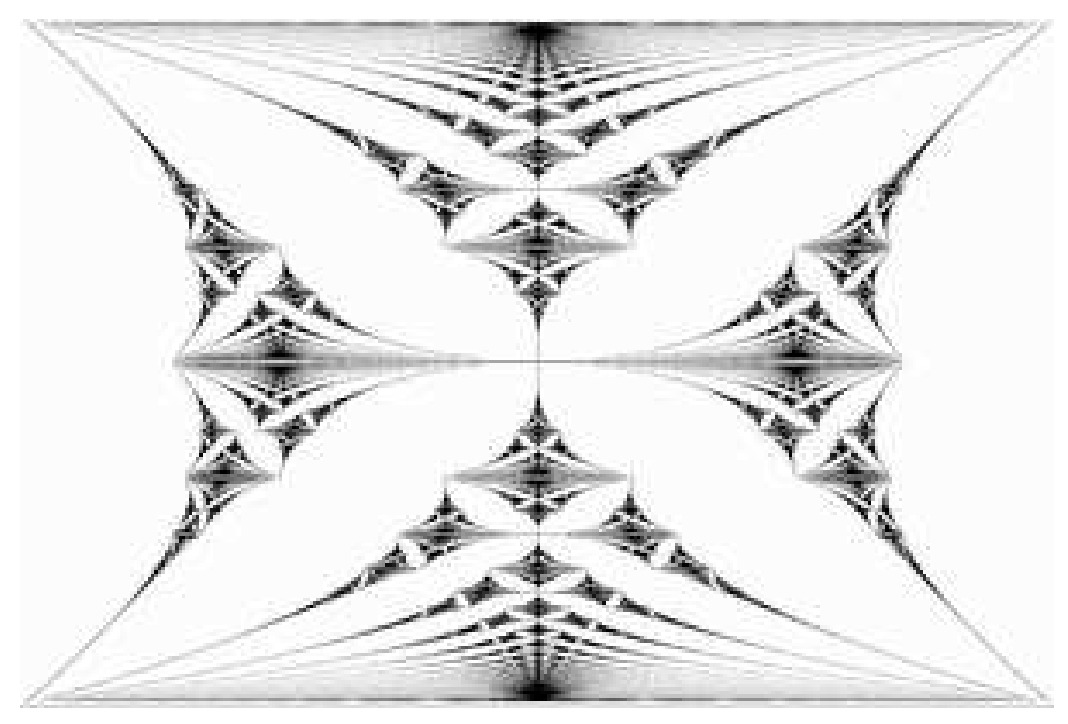

Figure 1: Band spectrum for a 2D Bloch electron in a uniform magnetic field

Even in a perfect crystal, the electronic motion, when submitted to a magnetic field, is no longer periodic [89]. The lack of periodicity is due to the quantum phase created by the magnetic field in the electronic wave functions that breaks the translation symmetry. The problem of computing the band spectrum for a Bloch electron in a magnetic field has been one of the most chalenging ones in Solid State Physics. The Peierls substitution [89] permits to reduce the problem to a tight-binding one, the most celebrated such a model being the Harper one [60]. It was only in 1976 that the spectrum of the Harper model could be computed by Hofstadter [63] thanks to the arrival of a new generation of computers (see Fig. 1). Another way to describe this aperiodicity is to see the magnetic field acting as an effective Planck constant that makes the ordinary space noncommutative from the point of view of quantum charged particles [13, 15, 95].

However, there are many materials that are not crystalline microscopically. The various varieties of glass are probably the most common examples. These materials are still a challenge for theoreticians. Less common, but easier to study, is the silicon that exhibits a crystalline phase with diamond lattice, and also an amorphous phase that is more like a glass than a crystal. A rather successful description of amorphous semiconductors was 
proposed by Sadoc et al. [72] in the early eighties, based upon saying that such a stucture looks like a perfect crystal in a curved space, only flattened to accomodate the flat $3 D$ space.

A serious need for investigating non crystalline solid came from semiconductors due to their importance for the electronic industry. For indeed, when pure, a semiconductor is an insulator due to a large gap at the Fermi level [104]. However, impurities, either created by artificially doping or spontaneously present in nature, provide enough electrons in the conduction band (or holes in the valence band) to make it a conductor as long as the temperature is large enough. At low temperature, however, the charge carriers are trapped in the impurity band and they see only the sublattice of impurities. Hence, from the point of view of charge carriers, the semiconductor looks like a random lattice, completely disordered. This problem became the focus of attention in the late seventies when the technology was ready to produce mesoscopic devices. Several phenomena like the Anderson localization, the enhancement of the magnetoresistance, the universality of quantum fluctuations, the quantum Hall effect, became the basic elements of such physics. One side consequence of the semiconductor technology of this time was the possibility of creating artificial structures, like superlattices, that may mimick situation proposed by theoreticians. For example, $1 D$ chains with potentials varying according to a prescribed rule, such as the Fibonacci or the Thue-Morse sequences can be created in this way.

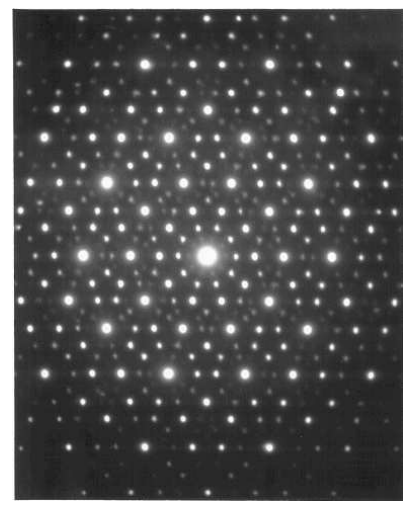

Figure 2: Diffraction picture of a quasicrystal

In 1984, a new class of materials, called quasicrystals was discovered [102]. The first sample was an alloy of aluminium and manganese. The surprise was that the diffraction spectrum obtained by transmission electron microscopy (T.E.M.) was pointlike, like in a perfect crystal, but exhibited a forbidden 5-fold symmetry (see Fig. 2). The solution proposed to this paradox was that the atomic arrangement was no longer periodic, but rather quasiperiodic in space. This implies a long range order of a new type.

Later, a large number of quasicrystalline alloys, mainly made with aluminium and mainly ternary, were produced [62]. The most important are $A l_{62.5} \mathrm{Cu}_{25} \mathrm{Fe}_{12.5}$ (where the iron con- 

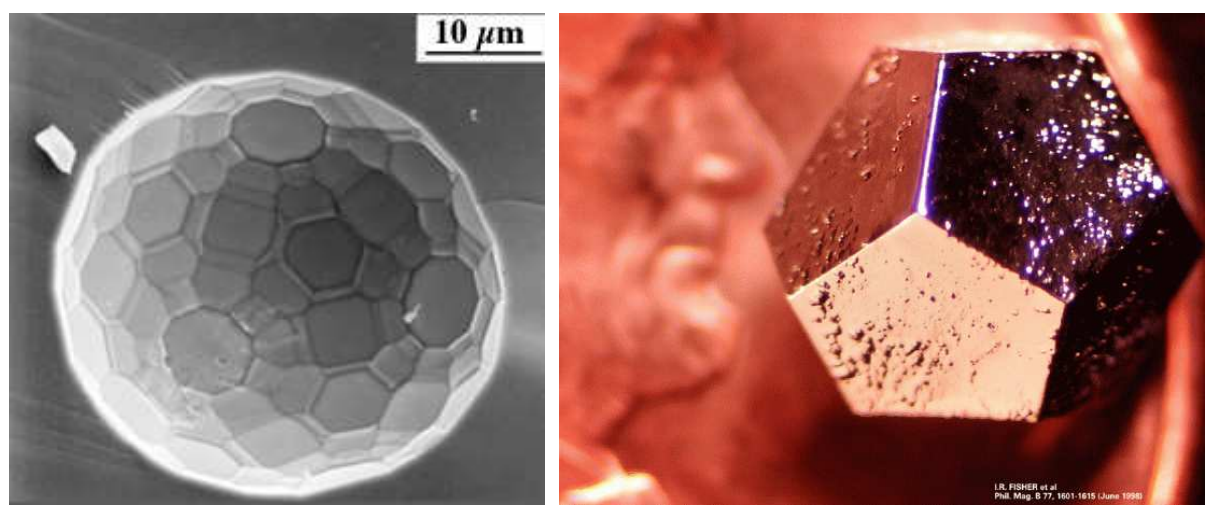

Figure 3: Left: Faceted hole in an Al-Mn-Pd quasicrystal [11]

Figure 4: Right: Dodecahedral single grain Ho-Mg-Zn quasicrystal [52]

centration may not vary more than .5\% to stay in the quasicristalline phase), $A l_{70} P d_{21} M n_{9}$ (see Fig. 3) or $A l_{70} P d_{21} R e_{9}$ due to the extreme quality of the samples that can be produced (the concentration of impurities or of defect is now less than $10^{-5}$ ). These materials may have mostly an icosahedral symmetry, like the $\mathrm{HoMgZn}_{\mathrm{Z}}$ monograin shown in Fig. 4, or a decagonal symmetry. But other compounds have been produced with other symmetries, like 8-fold or 12-fold symmetries. Besides having their surprising structure, these materials exhibit strange properties. For instance, even though made of good metals, they are mostly insulators at low temperature $[62,99]$. They are also mechanically hard and fragile, and they exhibit a superplastic transition at high temperature just below the melting temperature (see for instance [62] for some of these properties). From the point of view that is developped in this course, the most interesting aspect of these materials is that there is no way of treating the aperiodicity as a perturbation of a periodic structure. Moreover, numerical calculations proved to be extremely hard and they are not powerful enough to explain most of the properties described above. There is a need for a new approach and this a typical interesting problem of Mathematical Physics.

\subsection{The Hull}

The starting point of the theory consists in considering the set of atomic positions in the ideal case where the atoms are fixed at their equilibrium position at zero temperature. This is a set of points in the ambient space $\mathbb{R}^{d}$. This set will be considered in the idealized situation for which the solid has infinite volume and is homogeneous in space. This is a convenient approximation that fails only for submicronic samples. Let $\mathcal{L}$ denote this set. It is clear that atoms cannot be too close to each others, due to nucleus repulsion at short distance. Moreover, since the system is idealized at zero temperature, except for special situations, like for zeolites, no lacuna is expected to occur so that there is a maximal size 
to holes between these points. This can be axiomatized in the following way [76]

Definition 1 1)- $A$ subset $\mathcal{L}$ of $\mathbb{R}^{d}$ is uniformly discrete if there is $r>0$ such that every open ball of radius $r$ meets $\mathcal{L}$ at most on one point. Then $\mathcal{L}$ will be called $r$-discrete.

2)- $A$ subset $\mathcal{L}$ of $\mathbb{R}^{d}$ is relatively dense if there is $R>0$ such that every closed ball of radius $R$ meets $\mathcal{L}$ at least on one point. Then $\mathcal{L}$ will be called $R$-dense.

3)- A subset $\mathcal{L}$ of $\mathbb{R}^{d}$ is a Delone set (or also Delauney) if it is both uniformly discrete and relatively dense. $\mathcal{L}$ will be called $(r, R)$-Delone if it is $r$-discrete and $R$-dense.

4)- $A$ Delone set $\mathcal{L}$ has finite type whenever $\mathcal{L}-\mathcal{L}$ is locally finite

5)- A Delone set is a Meyer set whenever $\mathcal{L}-\mathcal{L}$ is itself a Delone set.

Example 1 1. A random subset $\mathcal{L}$ of $\mathbb{R}^{d}$ distributed according to the Poisson distribution with a finite positive density is almost surely discrete, but with probability one, it is neither uniformly discrete nor relatively dense.

2. Let $\mathcal{L}_{0}$ be a lattice in $\mathbb{R}^{d}$, namely a discrete cocompact subgroup of $\mathbb{R}^{d}$. Let $\mathcal{L}$ be a random subset of $\mathcal{L}_{0}$ distributed according to the Bernoulli law on each sites. Then with probability one $\mathcal{L}$ is uniformly discrete (obvious) but not relatively dense. This situation occurs precisely for the distribution of impurity sites in a lightly doped semiconductor [104].

3. Most solids are described, at zero temperature by Delone sets. This is the case for amorphous materials (silicon), glasses, crystals.

4. The cut-and-project method to describe quasicrystals [62] shows that the set of atomic sites of such a material is a Meyer set.

In order to represent a uniformly discrete set $\mathcal{L}$, it is convenient to consider its counting measure which is the Radon measure on $\mathbb{R}^{d}$

$$
\nu^{(\mathcal{L})}=\sum_{y \in \mathcal{L}} \delta(.-y)
$$

Recall that a Radon measure $\mu$ on $\mathbb{R}^{d}$ is an element of the dual space to the space $\mathcal{C}_{c}\left(\mathbb{R}^{d}\right)$ of continuous functions with compact support. Since it is not the aim of this paper to give all technical details, the reader should look at [71] to learn more about the natural topology on this space. Thus $\mu$ becomes a linear map $\mu: f \in \mathcal{C}_{c}\left(\mathbb{R}^{d}\right) \mapsto \mu(f) \in \mathbb{C}$ which is continuous. Here $\nu^{(\mathcal{L})}(f)=\sum_{y \in \mathcal{L}} f(y)$ and this sum is finite since $f$ has compact support and since $\mathcal{L}$ is discrete. The measure $\nu^{(\mathcal{L})}$ is a counting measure that is

Definition $2 A$ counting measure on $\mathbb{R}^{d}$ is a Radon measure $\nu$ such that any ball $B \subset \mathbb{R}^{d}$ has an integer measure, that is $\nu(B) \in \mathbb{N}$. This measure is $r$-discrete if for any open ball 
$B$ of radius less than or equal to $r$ then $\nu(B) \leq 1$. It is called $R$-dense if for any closed ball $B$ of radius larger than or equal to $R$ as a measure $\nu(B) \geq 1$. It is $(r, R)$-Delone if it is both $r$-discrete and $R$-dense.

It is not difficult to check that there is a one-to-one correspondance between discrete sets and counting measures, the set being the support of the measure, so that each property of such a set can be read on the associated measure and vice versa. Representing the atomic set by a measure is a convenient tool to describe topologies. The space $\mathfrak{M}\left(\mathbb{R}^{d}\right)$ of Radon measures on $\mathbb{R}^{d}$ will be endowed with the weak* topology over $\mathcal{C}_{c}\left(\mathbb{R}^{d}\right)$. This means that a sequence $\left(\mu_{n}\right)_{n \in \mathbb{N}}$ of Radon measures converges to $\mu$ if and only if given any continuous function $f$ with compact support on $\mathbb{R}^{d}$, then $\lim _{n \rightarrow \infty} \mu_{n}(f)=\mu(f)$. Thanks to this language, to say that a sequence $\mathcal{L}_{n}$ of discrete sets converges to the discrete set $\mathcal{L}$ means that in each open ball $B$ the sets $\mathcal{L}_{n} \cap B$ converges to $\mathcal{L} \cap B$, say for the Hausdorff distance. Note however, that this convergence needs not being uniform w.r.t. B. So it is convenient to denote by $U D_{r}\left(\mathbb{R}^{d}\right)$ the set of $r$-discrete counting Radon measures on $\mathbb{R}^{d}$. In very much the same way let $\operatorname{Del}_{r, R}\left(\mathbb{R}^{d}\right)$ be the set of $(r, R)$-Delone Radon measures on $\mathbb{R}^{d}$. Then [23]:

Proposition 1 1)- $U D_{r}\left(\mathbb{R}^{d}\right)$ and Del $l_{r, R}\left(\mathbb{R}^{d}\right)$ are closed and compact subspaces of $\mathfrak{M}\left(\mathbb{R}^{d}\right)$. 2)- The closure $Q D\left(\mathbb{R}^{d}\right)$ of the union $\bigcup_{r>0} U D_{r}\left(\mathbb{R}^{d}\right)$ in $\mathfrak{M}\left(\mathbb{R}^{d}\right)$, is the set of counting measures.

3)- $U D_{r}\left(\mathbb{R}^{d}\right)$ is the closure of the union $\bigcup_{R>r} D e l_{r, R}\left(\mathbb{R}^{d}\right)$.

Remark 1 1)- means that from any sequence of $r$-discrete sets it is possible to extract a convergent subsequence that converges to an $r$-discrete set. In particular the limit of a convergent sequence of $r$-discrete sets is itself $r$-discrete.

2 )- a measure of $Q D\left(\mathbb{R}^{d}\right)$ can be seen as an atomic set in which a finite number of atoms can sit on top of each other.

3 )- means that each $r$-discrete set can be approximated by a sequence of $r$-discrete Delone sets.

Remark 2 In much the same way, a Meyer set is an $(r, R)$-Delone set such that $\mathcal{L}-\mathcal{L}$ is $\left(r^{\prime}, R^{\prime}\right)$-Delone for some $0<r<R, 0<r^{\prime}<R^{\prime}$. If $M e y_{r, R ; r^{\prime}, R^{\prime}}\left(\mathbb{R}^{d}\right)$ denote such a set of measures, it is also compact in the weak* topology.

Remark 3 The property for $\mathcal{L}$ of having finite type is not preserved under limits.

Given now $a \in \mathbb{R}^{d}$, the translation $\mathrm{T}^{a}: x \in \mathbb{R}^{d} \mapsto x+a \in \mathbb{R}^{d}$ acts on $\mathcal{C}_{c}\left(\mathbb{R}^{d}\right)$ through $\mathrm{T}^{a} f(x)=f(x-a)$ whenever $f \in \mathcal{C}_{c}\left(\mathbb{R}^{d}\right)$. Therefore it also acts on the space of Radon measures through $\mathrm{T}^{a} \mu(f)=\mu\left(\mathrm{T}^{-a} f\right)$ whenever $f \in \mathcal{C}_{c}\left(\mathbb{R}^{d}\right)$ and $\mu \in \mathfrak{M}\left(\mathbb{R}^{d}\right)$. It is elementary to check that these maps are continuous and invertible. Hence the translation group $\mathbb{R}^{d}$ acts on $\mathfrak{M}\left(\mathbb{R}^{d}\right)$ in a continuous way and 
Proposition 2 The spaces $Q D\left(\mathbb{R}^{d}\right), U D_{r}\left(\mathbb{R}^{d}\right)$ and Del $l_{r, R}\left(\mathbb{R}^{d}\right)$ are $\mathbb{R}^{d}$-invariant.

Remark 4 In much the same way $M e y_{r, R ; r^{\prime}, R^{\prime}}\left(\mathbb{R}^{d}\right)$ is translation invariant.

Remark 5 If $\mathcal{L}$ has finite type, then all its translated have finite type.

This last result allows to define the Hull of a uniformly discrete set as follows

Definition 3 Let $\mathcal{L}$ be a uniformly discrete subset of $\mathbb{R}^{d}$. Then its Hull is the dynamical system $\left(\Omega, \mathbb{R}^{d}, \mathrm{~T}\right)$ where $\Omega$ is the closure of the $\mathbb{R}^{d}$-orbit of $\nu^{(\mathcal{L})}$ in $\mathfrak{M}\left(\mathbb{R}^{d}\right)$.

Remark 6 1)- Since $\mathcal{L}$ is uniformly discrete there is $r>0$ such that $\nu^{(\mathcal{L})} \in U D_{r}\left(\mathbb{R}^{d}\right)$. Hence, by Prop. 2 its orbit is contained in $U D_{r}\left(\mathbb{R}^{d}\right)$. By Prop. 1 then, $\Omega$ is a compact subset of $U D_{r}\left(\mathbb{R}^{d}\right)$. In particular, any measure $\omega \in \Omega$ defines an $r$-discrete set $\mathcal{L}_{\omega}$, namely its support.

2 )- The closure of the orbit of any point is obviously translation invariant, so that $\mathbb{R}^{d}$ acts on $\Omega$ through T.

3)- If in addition $\mathcal{L}$ is $(r, R)$-Delone, the same argument implies that all $\mathcal{L}_{\omega}$ 's are $(r, R)$ Delone.

4)- If $\mathcal{L}$ has finite type, then so does any element of the Hull. Actually then $\mathcal{L}_{\omega}-\mathcal{L}_{\omega} \subset \mathcal{L}-\mathcal{L}$ for any $\omega \in \Omega$. In particular if $\mathcal{L}$ is Meyer, so does any element of the Hull.

\subsection{Properties of the Hull}

Let $\mathcal{L}$ be a uniformly discrete set in $\mathbb{R}^{d}$ and let $\Omega$ be its Hull. Then the canonical transversal is the subset of $X \subset \Omega$ defined by

$$
X=\left\{\omega \in \Omega ; 0 \in \mathcal{L}_{\omega}\right\}=\{\omega \in \Omega ; \omega(\{0\})=1\} \quad \text { (Canonical transversal) }
$$

Each orbit meets this transversal on the corresponding atomic set, namely

$$
\mathrm{T}^{-x} \omega \in X \quad \Leftrightarrow \quad x \in \mathcal{L}_{\omega} .
$$

Since $\mathcal{L}$ is uniformly discrete, so is $\mathcal{L}_{\omega}$, so that there is a minimum distance from one point of $X$ to any other along the orbit. In this sense the orbits are transversal.

Example 2 Let $\mathcal{L}$ be periodic with period group $\mathbb{G}$. If $\mathbb{G}$ is a lattice, namely a discrete subgroup of $\mathbb{R}^{d}$ that generates $\mathbb{R}^{d}$ as a vector space, then the Hull is homeomorphic to the torus $\mathbb{R}^{d} / \mathbb{G}$. Moreover, the transversal is the finite set $X=\mathcal{L} / \mathbb{G}$. 
Example 3 A quasicrystal can be constructed by means of the cut-and-project method (see Section 3.3). For $N>d$, let $\mathcal{R}$ be a lattice in $\mathbb{R}^{N}$ and let $\Delta$ be a polyhedral fundamental domain. Let then $\mathcal{E}_{\|}$be a $d$-dimensional subspace of $\mathbb{R}^{N}$ meeting $\mathcal{R}$ only at the origin. Then project all points of the strip $\Delta+\mathcal{E}_{\|}$on $\mathcal{E}_{\|}$to get $\mathcal{L}$. By identifying $\mathcal{E}_{\|}$ with $\mathbb{R}^{d}, \mathcal{L}$ becomes a Meyer set that is a model for all known quasicrystals [62]. Let $W=$ $\pi_{\perp}(\Delta)$, where $\pi_{\perp}$ denote the projection operator on the orthogonal subspace of $\mathcal{E}_{\|}$. Let $W$ be endowed with the coarsest topology such that $W \cap\left(W+\pi_{\perp}\left(a_{1}\right)\right) \cap \cdots \cap\left(W+\pi_{\perp}\left(a_{n}\right)\right)$ becomes closed and open for any family $\left\{a_{1}, \cdots, a_{n}\right\} \subset \mathcal{R}$. Then $W$ is homeomorphic to the transversal [23].

Associated with the transversal $X$ of the Hull, is a groupoid $\Gamma(X)$ [96]. This groupoid plays a rôle similar to the notion of Poincaré map or first return map in the theory of dynamical systems $[42,69]$. This groupoid is defined as follows. The set of unit $\Gamma^{(0)}$ coincides with $X$. The set of arrows $\Gamma(X)$ is the set of pairs $(\omega, a) \in X \times \mathbb{R}^{d}$ such that $\mathrm{T}^{-a} \omega \in X$. Then the range, the source and the composition maps are defined by

$$
r(\omega, a)=\omega, \quad s(\omega, a)=\mathrm{T}^{-a} \omega, \quad(\omega, a) \circ\left(\mathrm{T}^{-a} \omega, b\right)=(\omega, a+b)
$$

The fiber $\Gamma^{(\omega)}$ is $r^{-1}(\{\omega\})$. Endowed with the topology induced by $\Omega \times \mathbb{R}^{d}$, this is a locally compact groupoid. If $\mathbb{P}$ is an $\mathbb{R}^{d}$-invariant ergodic measure on $\Omega$, then it induces on $\Gamma(X)$ a transverse measure represented by a probability measure $\mathbb{P}_{t r}$ on $X[36]$.

In the following it will be convenient to introduce the Hausdorff distance of two sets $A, B$ in $\mathbb{R}^{d}$, namely $d_{H}(A, B)=\max \left\{\sup _{x \in A} \inf _{y \in B}|x-y|, \sup _{y \in B} \inf _{x \in A}|x-y|\right\}$. The first property of such a system is given as follows [23]. It is necessary to recall that a dynamical system is minimal if every orbit is dense

Proposition 3 If $\mathcal{L}$ is uniformly discrete but not relatively dense, then $\Omega$ admits a fixpoint the orbit of which does not meet $X$. In particular the Hull is not minimal.

Consequently, $\mathcal{L}$ must be Delone to have a minimal Hull.

Definition 4 Let $\mathcal{L}$ be a Delone set in $\mathbb{R}^{d}$. Then $\mathcal{L}$ is repetitive if for any finite subset $p \subset \mathcal{L}$, and any $\epsilon>0$, there is $R>0$ such that any ball of radius $R$ contains a translated of a finite subset $p^{\prime}$ such that $d_{H}\left(p, p^{\prime}\right)<\epsilon$.

The following can be found in $[76,70,23]$

Theorem 1 Let $\mathcal{L}$ be a Delone set. Then its Hull is minimal if and only if it is repetitive.

From Def. 1 a uniformly discrete set $\mathcal{L}$ has finite type whenever $\mathcal{L}-\mathcal{L}$ is discrete, thus closed. In such case the following is true 
Proposition 4 A uniformly discrete subset $\mathcal{L}$ of $\mathbb{R}^{d}$ with finite type admits a Cantorian transversal.

A characterization of the Hull of a finite type repetitive Delone set has been given in [24] (see Section 3.4)

Theorem 2 Let $\mathcal{L}$ be a repetitive Delone set with finite type in $\mathbb{R}^{d}$. Then its Hull is conjugate by homeomorphisms to the projective limit of an inverse sequence of branched oriented flat compact manifolds without boundaries, in which the $\mathbb{R}^{d}$-action is induced by parallel transport of constant vector fields.

\subsection{Atomic Gibbs groundstates}

In realistic solids, the atomic positions are also determined by their thermodynamical properties. This is because atoms can vibrate around their equilibrium position and can also diffuse through the solid. This motion creates both acoustic waves (phonons) and lacunæ. Nevertheless, the atomic position can still be described by a discrete set, thus an element of $Q D\left(\mathbb{R}^{d}\right)$. Hence this last space plays the rôle of the configuration space that is needed in Statistical Mechanics. Nevertheless, it is very convenient to describe the atomic motion in solids as a perturbation of the equilibrium position. The acoustic waves are usually treated as phonon degrees of freedom, whereas lacunæ can be seen as impurities. Still, the atomic positions can be seen as typical configurations for the Gibbs measure describing the thermal equilibrium of atoms. Neglecting the atomic motion is equivalent to considering the $T \downarrow 0$ limit $\mathbb{P}$ of the Gibbs measure for the atoms. Then $\mathbb{P}$ can be seen as a probability measure on $Q D\left(\mathbb{R}^{d}\right)$.

From the mathematical point of view, there is a difficulty. For indeed, $Q D\left(\mathbb{R}^{d}\right)$ is not a locally compact space, so that the notion of Radon measure is meaningless. Nevertheless, the weak* topology makes this space a Polish space [71]. That is, the topology can be described through a distance for which the space is complete (the choice of such a distance is actually not unique and certainly not canonical). But the beauty of Polish spaces is that there is a genuine theory of probabilities [85] using the Borel approach through $\sigma$-additive functions on the $\sigma$-algebra of Borel sets. In particular, since the translation group acts on $Q D\left(\mathbb{R}^{d}\right)$ in a continuous way, it transforms Borel sets into Borel sets so that it also acts on the space of probabilities by $\mathrm{T}^{a} \mathbb{P}(A)=\mathbb{P}\left(\mathrm{T}^{-a} A\right)$ for $A$ a Borel set. The Prokhorov theorem gives also a very useful criterion for compactness of a family of such probabilities. It turns out that describing the atomic configurations through such probabilities gives rise to several interesting results for physicists [23].

If the solid under consideration is homogeneous, then its Gibbs measure $\mathbb{P}$ is expected to be translation invariant. Moreover, standard results of Statistical Mechanics [100] show that a translation invariant pure phase is described by a Gibbs measure that is ergodic under the translation group. In addition with such considerations, the analysis proposed 
in Section 2.2 shows that typical configurations of atoms at zero temperature should be at least uniformly discrete, but also Delone unless in very special cases. This is why the following definition can be useful

Definition 5 An atomic grounstate is a probability measure $\mathbb{P}$ on $Q D\left(\mathbb{R}^{d}\right)$ such that

1. $\mathbb{P}$ is $\mathbb{R}^{d}$-invariant;

2. $\mathbb{P}$ is $\mathbb{R}^{d}$-ergodic;

3. the space of uniformly discrete sets has $\mathbb{P}$-probability one.

In addition, $\mathbb{P}$ is called Delone (resp. Meyer) if it gives probability one to the space of Delone (resp. Meyer) sets.

Several results have been obtained in [23] from such a definition.

Theorem 3 Let $\mathbb{P}$ be an atomic groundstate. Then

1. there is $r>0$ such that $\mathbb{P}$-almost every atomic configuration $\mathcal{L}$ is $r$-discrete an not $r^{\prime}$-discrete for $r^{\prime}>r$;

2. there is a compact subset $\Omega \subset U D_{r}\left(\mathbb{R}^{d}\right)$ such that for $\mathbb{P}$-almost all atomic configuration $\mathcal{L}$, the Hull of $\mathcal{L}$ is $\Omega ; \Omega$ coincides with the topological support of $\mathbb{P}$;

3. if, in addition, $\mathbb{P}$ is Delone (resp. Meyer), there is a unique pair $(r, R)$ (resp. family $\left.\left(r, R ; r^{\prime}, R^{\prime}\right)\right)$ such that $\mathbb{P}$-almost every configuration is $(r, R)$-Delone and not $\left(r ", R\right.$ ")-Delone for $r<r "$ and $R$ " $<R$ (resp. $\left(r, R ; r^{\prime}, R^{\prime}\right)$-Meyer and not $\left(r_{1}, R_{1} ; r_{1}^{\prime}, R_{1}^{\prime}\right)$ for $\left.r<r_{1}, R>R_{1}, r^{\prime}<r_{1}^{\prime}, R^{\prime}>R_{1}^{\prime}\right)$.

The next result concerns the notion of diffraction measure. Let $\mathcal{L}$ be a point set representing the position of atoms in the solid. Then, the diffraction pattern seen on a screen, in an $X$-ray diffraction experiment or in a transmission electronic microscope (T.E.M.), can be computed from the Fourier transform of $\mathcal{L}$ restricted to the domain $\Lambda$ occupied by the sample in $\mathbb{R}^{d}$. More precisely, the intensity seen on the screen is proportional to

$$
I_{\Lambda}(k)=\frac{1}{|\Lambda|}\left|\sum_{x \in \mathcal{L} \cap \Lambda} e^{\imath\langle k \mid x\rangle}\right|^{2}
$$

where $k \in \mathbb{R}^{d}$ represents the wave vector of the diffraction beam, the direction of which gives the position on the screen. The Fourier transform of $I_{\Lambda}(k)$ is given by the following expression: if $f \in \mathcal{C}_{c}\left(\mathbb{R}^{d}\right)$, with Fourier transform denoted by $\tilde{f}$, then

$$
\int_{k \in \mathbb{R}^{d}} d k \tilde{f}(k) I_{\Lambda}(k)=\frac{1}{|\Lambda|} \sum_{x, y \in \mathcal{L} \cap \Lambda} f(x-y)=\rho_{\mathcal{L}}^{(\Lambda)}(f)
$$


where $\rho_{\mathcal{L}}^{(\Lambda)}$ will be called the finite volume diffraction measure. From eq. (4), it follows that $\rho_{\mathcal{L}}^{(\Lambda)} \in \mathfrak{M}\left(\mathbb{R}^{d}\right)$ is a positive measure with a Fourier transform being also a positive measure. The main problem is whether such a quantity converges as $\Lambda \uparrow \mathbb{R}^{d}$. The next theorem gives conditions under which convergence holds

Theorem 4 Let $\mathbb{P}$ be an atomic groundstate. Then:

(i) For $\mathbb{P}$-almost every $\mathcal{L}$ the family $\rho_{\mathcal{L}}^{(\Lambda)}$ of measures on $\mathbb{R}^{d}$ converges to a positive measure $\rho_{\mathbb{P}} \in \mathfrak{M}\left(\mathbb{R}^{d}\right)$.

(ii) The distributional Fourier transform of $\rho_{\mathbb{P}}$ is also a positive measure on $\mathbb{R}^{d}$.

In other words, $\mathbb{P}$ determines in a unique way the diffraction pattern.

\subsection{Bloch Theory}

If the solid is a perfect crystal, the set $\mathcal{L}$ is invariant under a translation group $\mathbb{G}$. $\mathbb{G}$ is a lattice in $\mathbb{R}^{d}$ namely a discrete subgroup generating $\mathbb{R}^{d}$ as a vector space. $\mathbb{G}$ is called the period group of $\mathcal{L}$. Bloch theory deals with the Schrödinger equation with a $\mathbb{G}$-periodic potential. More precisely, let $\mathcal{H}=L^{2}\left(\mathbb{R}^{d}\right)$ be the Hilbert space of quantum states. The groups $\mathbb{G}$ is unitarily represented in $\mathcal{H}$ through $U(a) \psi(x)=\psi(x-a)$ whenever $a \in \mathbb{G}$. The Schrödinger operator is a selfadjoint operator $H=H^{*}=-\Delta+V$ with dense domain, where $\Delta$ is the Laplacian on $\mathbb{R}^{d}$ and $V$ is a locally $L^{1}, \mathbb{G}$-periodic real valued function. In particular

$$
U(a) H U(a)^{-1}=H \quad \forall a \in \mathbb{G} .
$$

Therefore $H$ and the $U(a)$ 's can be simultaneously diagonalized. Since $\mathbb{G}$ is Abelian, diagonalization of the $U(a)$ 's is performed through its character group $\mathbb{G}^{*}$. Standard results in Pontryagin duality theory imply that $\mathbb{G}^{*}$ is isomorphic to the quotient $\mathbb{B}=$ $\mathbb{R}^{d *} / \mathbb{G}^{\perp}$ of the dual group of $\mathbb{R}^{d}$ (isomorphic to $\mathbb{R}^{d}$ ) by the orthogonal $\mathbb{G}^{\perp}$ of $\mathbb{G}$ in this group. It is a well known fact that $\mathbb{G}^{\perp}$ is a lattice in $\mathbb{R}^{d}$ (called the reciprocal lattice in Solid State Physics [65]) so that $\mathbb{B}=\mathbb{R}^{d *} / \mathbb{G}^{\perp}$ is a compact group homeomorphic to a $d$-torus. $\mathbb{B}$ will be called the Brillouin zone (strictly speaking this is slightly different from what crystallographers call Brillouin zone).

The concrete calculation of $\mathbb{B}$ goes as follows: any character of $\mathbb{R}^{d}$ is represented by an element $k \in \mathbb{R}^{d *}$. Since $\mathbb{R}^{d *}$ and $\mathbb{R}^{d}$ can be identified canonically, by using the usual Euclidean structure, $k$ can be seen as a vector $k=\left(k_{1}, \cdots, k_{d}\right) \in \mathbb{R}^{d}$. The corresponding character is given by the map

$$
\eta_{k}: x \in \mathbb{R}^{d} \mapsto e^{\imath\langle k \mid x\rangle} \in U(1), \quad\langle k \mid x\rangle=k_{1} x_{1}+\cdots+k_{d} x_{d} .
$$

In particular $\eta_{k}$ restricts to a character of $\mathbb{G}$, with the condition that $\left.\eta_{k}\right|_{\mathbb{G}}=\left.\eta_{k^{\prime}}\right|_{\mathbb{G}}$ if and only if $k-k^{\prime} \in \mathbb{G}^{\perp}$, where 


$$
\mathbb{G}^{\perp}=\left\{b \in \mathbb{R}^{d} ;\langle b \mid a\rangle \in 2 \pi \mathbb{Z}, \forall a \in \mathbb{G}\right\} .
$$

Since $\mathbb{B}$ is a compact group, the diagonalization of the $U(a)$ 's requires the use of a direct integral decomposition of $\mathcal{H}$ over $\mathbb{B}$, so that

$$
\mathcal{H}=\int_{k \in \mathbb{B}}^{\oplus} d^{d} k \mathcal{H}_{k} \quad H=\int_{k \in \mathbb{B}}^{\oplus} d^{d} k H_{k} .
$$

Here, $\mathcal{H}_{k}$ is the space of measurable functions $\psi$ on $\mathbb{R}^{d}$ such that $\psi(x+a)=e^{\imath\langle k \mid x\rangle} \psi(x)$ for all $a \in \mathbb{G}$ and that $\int_{\mathbb{V}} d^{d} x|\psi(x)|^{2}=\|\psi\|_{\mathcal{H}_{k}}^{2}<\infty$, where $\mathbb{V}=\mathbb{R}^{d} / \mathbb{G}$. $H_{k}$ is then the partial differential operator formally given by the same expression as $H$, but with domain $\mathcal{D}_{k}$ given by the space of elements $\psi \in \mathcal{H}_{k}$ such that $\partial_{i} \psi / \partial x_{i} \in \mathcal{H}_{k}$, for $1 \leq i \leq d$, and $\Delta_{x} \psi \in \mathcal{H}_{k}$. Then $H_{k}$ is unitarily equivalent to an elliptic operator on the torus $\mathbb{R}^{d} / \mathbb{G}=\mathbb{V}$. (In solid state physics, $\mathbb{V}$ is called the Wigner-Seitz cell, whereas it is called the Voronoi cell in tiling theory.) Consequently, for each $k \in \mathbb{B}$, the spectrum of $H_{k}$ is discrete and bounded from below. If $E_{i}(k)$ denotes the eigenvalues, with a convenient labelling $i$, the maps $k \in \mathbb{B} \mapsto E_{i}(k) \in \mathbb{R}$ are called the band functions. The spectrum of $H$ is recovered

as $\operatorname{Sp}(H)=\bigcup_{i, k \in \mathbb{B}} E_{i}(k)$ and is called a band spectrum. A discrete spectrum is usually liable to be computable by suitable algorithms, since it restricts to diagonalizing large matrices.

This is a short summary of Bloch theory. Strutt first realized the existence of band functions [107], but soon after Bloch wrote his important paper [27]. In 1930, Peierls gave a perturbative treatment of the band calculations [88] and Brillouin discussed the $2 D$ and $3 D$ cases [30]. The reader is invited to look at $[65,5]$ to understand why this theory has been so successful in solid state physics. Let us simply mention that the first explicit calculations of bands in 3D were performed in 1933 by Wigner \& Seitz [115] on sodium using the cellular method that holds their names. The symmetry properties of the wave function were explicitly used in an important paper by Bouckaert, Smoluchowski \& Wigner [29] leading to noticeable simplifications of the band calculation.

\subsection{The Noncommutative Brillouin zone}

In Section 2.2 it has been shown that an aperiodic solid is well described by its Hull $\left(\Omega, \mathbb{R}^{d}, \mathrm{~T}\right)$, namely a dynamical system with group $\mathbb{R}^{d}$ acting by homeomorphisms on a compact metrizable space $\Omega$. With any dynamical system, there is a canonical $C^{*}$-algebra namely the crossed product $\mathcal{C}(\Omega) \rtimes \mathbb{R}^{d}[87]$. In a similar way, such a system can also be described through its canonical transversal $X$, and its related groupoid $\Gamma(X)$. With any locally compact groupoid $\Gamma$, endowed with a transverse function [36], and with any module $\delta$ on $\Gamma$, is associated a $C^{*}$-algebra $C^{*}(\Gamma, \delta)[96]$. In this section, it will be shown that, after a slight modification if the solid is submitted to a magnetic field, $\mathcal{C}(\Omega) \rtimes \mathbb{R}^{d}$ is the smallest $C^{*}$-algebra generated by the electronic Schrödinger operator and all its translated. Moreover, it will be shown that, $C^{*}(\Gamma(X))$ is also generated by the matrix 
of phonon modes that appears in the equations of motion for phonons or by the effective Hamiltonian derived form the so-called tight binding representation $[13,16]$. It will also be shown that, for periodic crystals, this algebra is nothing but the set of continuous functions on the Brillouin zone.

Given a uniform magnetic field $B=\left(B_{\nu \mu}\right)$, namely a real-valued antisymmetric $d \times d$ matrix, the $C^{*}$-algebra $C^{*}\left(\Omega \times \mathbb{R}^{d}, B\right)$ is defined as follows. Let $\mathcal{A}_{0}$ be the topological vector space $\mathcal{C}_{c}\left(\Omega \times \mathbb{R}^{d}\right)$ of continuous functions with compact support in $\Omega \times \mathbb{R}^{d}$. It becomes a topological *-algebra when endowed with the following structure

$$
\begin{aligned}
f g(\omega, x) & =\int_{\mathbb{R}^{d}} d y f(\omega, y) g\left(T^{-y} \omega, x-y\right) e^{i \pi(e / h) B \cdot x \wedge y}, \\
f^{*}(\omega, x) & =\overline{f\left(T^{-x} \omega,-x\right)}
\end{aligned}
$$

where $f, g \in C_{c}\left(\Omega \times \mathbb{R}^{d}\right), B \cdot x \wedge y=\sum B_{\nu \mu} x_{\nu} y_{\mu}$ and $\omega \in \Omega, x \in \mathbb{R}^{d}$. Here $e$ is the electric charge of the particle and $h=2 \pi \hbar$ is Planck's constant. This *-algebra is represented on $L^{2}\left(\mathbb{R}^{d}\right)$ by the family of representations $\left\{\pi_{\omega} ; \omega \in \Omega\right\}$ given by

$$
\pi_{\omega}(f) \psi(x)=\int_{\mathbb{R}^{d}} d y f\left(T^{-x} \omega, y-x\right) e^{-i \pi(e / h) B \cdot x \wedge y} \psi(y), \quad \psi \in L^{2}\left(\mathbb{R}^{d}\right),
$$

where $\pi_{\omega}$ is linear, $\pi_{\omega}(f g)=\pi_{\omega}(f) \pi_{\omega}(g)$ and $\pi_{\omega}(f)^{*}=\pi_{\omega}\left(f^{*}\right)$. In addition $\pi_{\omega}(f)$ is a bounded operator and the representations $\left(\pi_{\omega}\right)_{\omega \in \Omega}$ are related by the covariance condition:

$$
U(a) \pi_{\omega}(f) U(a)^{-1}=\pi_{\mathrm{T}^{a} \omega}(f)
$$

where the U's are the so-called magnetic translations [119] defined by:

$$
U(a) \psi(x)=\exp \left\{(i e / \hbar) \int_{[x-a, x]} d y^{\mu} A_{\mu}(y)\right\} \psi(x-a),
$$

where $\vec{A}=\left(A_{1}, \ldots, A_{d}\right)$ is a vector potential defined by $B_{\mu \nu}=\partial_{\mu} A_{\nu}-\partial_{\nu} A_{\mu}, a \in \mathbb{R}^{d}$, $\psi \in L^{2}\left(\mathbb{R}^{d}\right)$ and $[x-a, x]$ is the line segment joining $x-a$ to $a$ in $\mathbb{R}^{d}$. A $C^{*}$-norm on $\mathcal{A}_{0}$ is defined by

$$
\|f\|=\sup _{\omega \in \Omega}\left\|\pi_{\omega}(f)\right\| .
$$

Definition 6 The noncommutative Brillouin zone is the topological manifold associated with the $C^{*}$-algebra $\mathcal{A}=C^{*}\left(\Omega \rtimes \mathbb{R}^{d}, B\right)$ obtained by completion of $\mathcal{A}_{0}=\mathcal{C}_{c}\left(\Omega \times \mathbb{R}^{d}\right)$ under the norm $\|\cdot\|$ defined by eq. (10).

For $B=0$ this construction gives the definition of the $C^{*}$-crossed product $\mathcal{C}(\Omega) \rtimes \mathbb{R}^{d}$ $[87,26]$. In the case of a perfect crystal (see Section 2.5), with lattice translation group $\mathbb{G}$, the hull $\Omega=\mathbb{R}^{d} / \mathbb{G}$ is homeomorphic to $\mathbb{T}^{d}$ (see Example 2 ) and 
Theorem $5[98,16]$ The $C^{*}$-algebra $C^{*}\left(\mathbb{R}^{d} / \mathbb{G} \rtimes \mathbb{R}^{d}, B=0\right)$ associated with a perfect crystal with lattice translation group $\mathbb{G}$, is isomorphic to $\mathcal{C}(\mathbb{B}) \otimes \mathcal{K}$, where $\mathcal{C}(\mathbb{B})$ is the space of continuous functions over the Brillouin zone and $\mathcal{K}$ the algebra of compact operators.

Even though the algebra $\mathcal{C}(\mathbb{B}) \otimes \mathcal{K}$ is already noncommutative, its noncommutativity comes from $\mathcal{K}$, the smallest $C^{*}$-algebra generated by finite rank matrices. It describes the possible vector bundles over $\mathbb{B}$. Theorem 5 is the reason to claim that $\mathcal{A}$ generalizes the Brillouin zone for aperiodic systems.

The groupoid $C^{*}$-algebra of $\Gamma(X)$ can be defined very similarly. Here $\mathcal{B}_{0}=\mathcal{C}_{c}(\Gamma(X))$ and the structure of ${ }^{*}$-algebra is given by

$$
\begin{aligned}
f g(\omega, x) & =\sum_{y \in \Gamma^{(\omega)}} f(\omega, y) g\left(T^{-y} \omega, x-y\right) e^{i \pi(e / h) B \cdot x \wedge y}, \\
f^{*}(\omega, x) & =\overline{f\left(T^{-x} \omega,-x\right)}
\end{aligned}
$$

Setting $\mathfrak{H}_{\omega}=\ell^{2}\left(\Gamma^{(\omega)}\right)$, there is a representation $\widehat{\pi}_{\omega}$ on $\mathfrak{H}_{\omega}$ defined by

$$
\widehat{\pi}_{\omega}(f) \psi(x)=\sum_{y \in \Gamma^{(\omega)}} f\left(T^{-x} \omega, y-x\right) e^{-i \pi(e / h) B \cdot x \wedge y} \psi(y), \quad \psi \in \mathfrak{H}_{\omega},
$$

Giving $\gamma=(\omega, a) \in \Gamma(X)$, there is a unitary operator $U(\gamma): \mathfrak{H}_{\mathrm{T}^{-a} \omega} \mapsto \mathfrak{H}_{\omega}$ defined by

$$
U(\gamma) \psi(x)=\exp \left\{(i e / \hbar) \int_{[x-a, x]} d y^{\mu} A_{\mu}(y)\right\} \psi(x-a), \quad \psi \in \mathfrak{H}_{\mathrm{T}^{-a} \omega},
$$

such that the covariance condition holds

$$
U(\gamma) \widehat{\pi}_{\mathrm{T}^{-a} \omega}(f) U(\gamma)^{-1}=\widehat{\pi}_{\omega}(f) .
$$

A $C^{*}$-norm on $\mathcal{B}_{0}$ is defined by

$$
\|f\|=\sup _{\omega \in X}\left\|\widehat{\pi}_{\omega}(f)\right\| .
$$

The $C^{*}$-algebra $\mathcal{B}=C^{*}(\Gamma(X), B)$ is the completion of $\mathcal{B}_{0}$ under this norm. The notion of Morita equivalence, quickly defined below, is defined in Section 4.1.1 (see Definition 13). The main result is the following [98]

Theorem 6 1)- The $C^{*}$-algebras $\mathcal{B}=C^{*}(\Gamma(X), B)$ and $\mathcal{A}=C^{*}\left(\Omega \rtimes \mathbb{R}^{d}, B\right)$ are Morita equivalent, namely $\mathcal{A}$ is isomorphic to $\mathcal{B} \otimes \mathcal{K}$.

2)- For $B=0$ and for a crystal $\mathcal{L}$ with period group $\mathbb{G}$ and transversal $X=\mathcal{L} / \mathbb{G}$, the $C^{*}$-algebra $\mathcal{B}$ is isomorphic to $\mathcal{C}(\mathbb{B}) \otimes M_{N}(\mathbb{C})$ if $N$ is the cardinality of $X$. 
In order to see the noncommutative Brillouin zone as a manifold, rules of Calculus are required. Integration is provided by using a transverse measure. Let $\mathbb{P}$ be an $\mathbb{R}^{d}$-invariant ergodic probability measure on $\mathbb{R}^{d}$ and let $\mathbb{P}_{t r}$ be the probability induced on $X$. Then traces are defined on $\mathcal{A}$ and $\mathcal{B}$ as follows

$$
\mathcal{T}_{\mathbb{P}}(f)=\int_{\Omega} d \mathbb{P}(\omega) f(\omega, 0), \quad f \in \mathcal{A}_{0}, \quad \hat{\mathcal{T}}_{\mathbb{P}}(f)=\int_{X} d \mathbb{P}_{t r}(\omega) f(\omega, 0), \quad f \in \mathcal{B}_{0},
$$

$\mathcal{T}_{\mathbb{P}}$ and $\hat{\mathcal{T}}_{\mathbb{P}}$ are traces in the sense that $\mathcal{T}_{\mathbb{P}}(f g)=\mathcal{T}_{\mathbb{P}}(g f)$, and that $\mathcal{T}_{\mathbb{P}}\left(f f^{*}\right)>0$. Whereas $\mathcal{T}_{\mathbb{P}}$ is not bounded, $\hat{\mathcal{T}}_{\mathbb{P}}$ is actually normalized, namely $\hat{\mathcal{T}}_{\mathbb{P}}(\mathbf{1})=1$. Moreover, using the Birkhoff ergodic theorem $[42,69]$, these traces can be seen as trace per unit volume in the following sense

$$
\mathcal{T}_{\mathbb{P}}(f)=\lim _{R \uparrow \infty} \frac{1}{|B(0 ; R)|} \operatorname{Tr}\left(\pi_{\omega}(f)\left\lceil_{B(0 ; R)}\right),\right.
$$

where $B(x ; R)$ denotes the ball of radius $R$ centered at $x \in \mathbb{R}^{d}$. A similar formula holds for $\hat{\mathcal{T}}_{\mathbb{P}}$ provided $|B(0 ; R)|$ is replaced by the number of points in $\mathcal{L}_{\omega} \cap B(0 ; R)$. In particular in the crystalline case,

$$
\mathcal{T}_{\mathbb{P}}(f)=\int_{\mathbb{B}} d k \operatorname{Tr}(\tilde{f}(k))
$$

if $\tilde{f}(k)$ denotes the representative of $f$ in $\mathcal{C}(\mathbb{B}) \otimes \mathcal{K}$ and $d k$ is the normalized Haar measure on $\mathbb{B} \simeq \mathbb{T}^{d}$. Therefore, these traces appears as the noncommutative analog of the integration over the Brillouin zone.

A positive measure on a topological space defines various spaces of measurable functions, such as the $L^{p}$-spaces. In much the same way, a positive trace on a $C^{*}$-algebra defines also $L^{p}$-spaces [101]. Whenever $1 \leq p<\infty, L^{p}\left(\mathcal{A}, \mathcal{T}_{\mathbb{P}}\right)$ is the separation-completion of $\mathcal{A}_{0}$ with respect to the seminorm $\|A\|_{p}=\mathcal{T}_{\mathbb{P}}\left\{\left(A^{*} A\right)^{p / 2}\right\}^{1 / p}$ for $A \in \mathcal{A}_{0}$. A similar definition is given for $\mathcal{B}$. In particular, $L^{2}\left(\mathcal{A}, \mathcal{T}_{\mathbb{P}}\right)$ coincides with the Hibert space of the GNSrepresentation $\pi_{G N S}$ of $\mathcal{A}$ associated with $\mathcal{T}_{\mathbb{P}}[45,108]$. Then $L^{\infty}\left(\mathcal{A}, \mathcal{T}_{\mathbb{P}}\right)$ denotes the von Neumann algebra generated by (namely the weak closure of) $\pi_{G N S}(\mathcal{A})$.

Similarly, a differential structure is provided in the following way

$$
\vec{\nabla} f(\omega, x)=\imath \vec{x} f(\omega, x), \quad f \in \mathcal{A}_{0} \text { or } \mathcal{B}_{0} .
$$

Here $\vec{x}$ denotes the vector $x \in \mathbb{R}^{d}$. It is easy to check that $\vec{\nabla}$ defines a ${ }^{*}$-derivation namely it obeys Leibniz rule $\vec{\nabla}(f g)=\vec{\nabla}(f) g+f \vec{\nabla}(g)$ and $\vec{\nabla}(f)^{*}=\vec{\nabla}\left(f^{*}\right)$. Moreover $\vec{\nabla}$ is the infinitesimal generator of a norm pointwise continuous $d$-parameter group of automorphisms defined by [87]

$$
\eta_{\vec{k}}(f)(\omega, x)=e^{\imath \vec{k} \cdot \vec{x}} f(\omega, x), \quad f \in \mathcal{A}_{0} \text { or } \mathcal{B}_{0}
$$


Let $\vec{X}$ be the position operator on $L^{2}\left(\mathbb{R}^{d}\right)$, (resp. on $\mathfrak{H}_{\omega}$ ), defined by $\vec{X} \psi(x)=\vec{x} \psi(x)$. Then

$$
\pi_{\omega}(\vec{\nabla} f)=\imath\left[\vec{X}, \pi_{\omega}(f)\right], \quad f \in \mathcal{A}_{0}, \quad \widehat{\pi}_{\omega}(\vec{\nabla} f)=\imath\left[\vec{X}, \widehat{\pi}_{\omega}(f)\right], \quad f \in \mathcal{B}_{0}
$$

In the crystalline case, it is easy to show that $\vec{\nabla}$ coincides with $\partial / \partial k$ in $\mathbb{B}[16]$. Hence $\vec{\nabla}$ appears as the noncommutative analog of the derivation in momentum space.

In the philosophy of A.Connes, a noncommutative Geometry for a compact manifold is given by a spectral triplet $(\mathfrak{A}, \mathcal{H}, D)$, where $\mathfrak{A}$ is a dense subalgebra of a unital $C^{*}$-algebra, invariant by holomorphic functional calculus, $\mathcal{H}$ a $\mathbb{Z}_{2}$-graded Hilbert space on which $\mathfrak{A}$ is represented by degree 0 operators and $D$ is a degree 1 selfadjoint operator with compact resolvent, such that $[D, A] \in \mathcal{B}(\mathcal{H})$ for all $A \in \mathfrak{A}$. Then $D$ plays the rôle of a Dirac operator on the noncommutative space associated with $\mathfrak{A}$, giving both a differential structure and a Riemannian metric corresponding to $d s^{2}=D^{-2}$ [41]. Then the dimension spectrum is provided by the set of poles of the $\zeta$-function

$$
\zeta_{D}(s)=\operatorname{Tr}\left(\frac{1}{|D|^{s}}\right)
$$

If $s_{0}$ denote the maximum real pole, then the residue at $s_{0}$ provides an integral over the manifold given by

$$
\int T=\lim _{s \downarrow s_{0}}\left(s-s_{0}\right) \operatorname{Tr}\left(\frac{1}{|D|^{s}} T\right)=\operatorname{Tr}_{\text {Dix }}\left(\frac{1}{|D|^{s_{0}}} T\right),
$$

where $\operatorname{Tr}_{\text {Dix }}$ denotes a Dixmier trace on $\mathcal{H}[41,44]$ (The definition and properties of the Dixmier trace will be given in Section 5.3). Such a structure is also present on the noncommutative Brillouin zone $\mathcal{B}$. For indeed, let $\gamma_{1}, \cdots, \gamma_{d}$ be an irreducible representation of the Clifford algebra of $\mathbb{R}^{d}$ by Dirac matrices satisfying $\gamma_{\mu} \gamma_{\nu}+\gamma_{\nu} \gamma_{\mu}=2 \delta_{\mu, \nu}$ and $\gamma_{\mu}^{*}=\gamma_{\mu}$ in the finite dimensional Hilbert space $\operatorname{Cliff}(d)$. Then $\operatorname{Cliff}(d)$ is graded by the matrix $\gamma_{0}=\gamma_{1} \gamma_{2} \cdots \gamma_{d}$, so that setting $\mathcal{H}=\mathfrak{H}_{\omega} \otimes \operatorname{Cliff}(d), G=\mathbf{1} \otimes \gamma_{0}$, this gives a graded Hilbert space where $G$ defines the graduation. Moreover $\mathfrak{A}=\mathcal{B}_{0}$ and $D=\sum_{\mu=1}^{d} \gamma_{\mu} X_{\mu}$ give a spectral triplet for which $s_{0}=d$. In particular, the Dixmier trace $\operatorname{Tr}_{\text {Dix }}\left(|D|^{-d}\right)$ exist for $\mathbb{P}_{t r}$-almost every $\omega \in X$ and its common value is given by

$$
\operatorname{Tr}_{\text {Dix }}\left(|D|^{-d}\right)=\operatorname{Dens}_{\mathbb{P}}\left(\mathcal{L}_{\omega}\right) \frac{\pi^{d}}{\Gamma(1+d / 2)},
$$

where $\operatorname{Dens}_{\mathbb{P}}\left(\mathcal{L}_{\omega}\right)$ is the density of $\mathcal{L}_{\omega}$ which exists for $\mathbb{P}$-almost all $\omega$ 's (see [23] see proof of Theorem 1.12). The numerical factor represents the volume of the unit ball of $\mathbb{R}^{d}$. Moreover, if $f \in \mathcal{B}_{0}$ this gives 


$$
\int f=\operatorname{Tr}_{\text {Dix }}\left(|D|^{-d} \widehat{\pi}_{\omega}(f)\right)=\hat{\mathcal{T}}_{\mathbb{P}}(f) \frac{\pi^{d}}{\Gamma(1+d / 2)}, \quad \mathbb{P}_{t r}-\text { a.e. } \omega \in X,
$$

showing that Connes' integral and the integral over the Brillouin zone coincide modulo normalization.

\subsection{Electrons and Phonons}

The formalism developped in the previous sections will eventually be useful to describe the quantum motion of electrons and phonons in an aperiodic solid, within the one particle approximation. The interacting case is more involved [105] and will not been considered in these notes.

The quantum motion of an electron in a monoatomic aperiodic solid represented by a uniformly discrete set $\mathcal{L}$ of atomic positions, and submitted to a uniform magnetic field $B$, is well described by a covariant family of Schrödinger operators of the type

$$
H_{\omega}=\frac{1}{2 m}\left(\frac{\hbar}{\imath} \vec{\nabla}-e \vec{A}\right)^{2}+\sum_{y \in \mathcal{L}_{\omega}} v(X-y), \quad \text { on } L^{2}\left(\mathbb{R}^{d}\right),
$$

where $\hbar$ is the Planck constant, $m$ is the electron mass, $\vec{A}$ is a vector potential and $v$ is an effective atomic potential, that represents the binding forces acting on the valence electron. In practice, an aperiodic solid contains more than one species of atoms, so that $\mathcal{L}$ must be replaced by a family $\mathcal{L}^{1}, \cdots, \mathcal{L}^{r}$ of uniformly discrete subsets representing the position of each atomic species, supposing that $r$ species are present. Then each species $i$ acts via an effective potential $v_{i}$. Moreover, the number of valence electrons involved in the conduction may be more than one per atom, so that it should be necessary to consider instead an $l$-body problem if $l$ valence electrons per atom are involved. This latter case will not be considered either, even though its treatment does not represent a major difficulty. In these notes the model described in eq. (19) will be sufficient.

The Schrödinger operator (19) is not well defined as long as no assumption is given on the nature of the atomic potential $v$. For the sum over the atomic sites to converge, it is necessary that $v$ decay fast enough at infinity. Also local regularity is necessary. A sufficient condition has been given in [23] (Lemma 2.22), namely let

$$
L_{K, r}^{1}\left(\mathbb{R}^{d}\right)=\left\{f \in L^{1}\left(\mathbb{R}^{d}\right) ;|f(x)| \leq \frac{K}{r^{d}} \int_{|x-y|<r / 2} d^{d} y|f(y)|, \text { for a.e. } \mathrm{x}\right\}
$$

be the set of integrable K-subharmonic functions on $\mathbb{R}^{d}$. Then

Lemma 1 Let $v \in L_{K, r}^{1}\left(\mathbb{R}^{d}\right)$ and $\nu \in U D_{r}\left(\mathbb{R}^{d}\right)$. Then $\nu * v \in L_{\mathbb{R}}^{\infty}\left(\mathbb{R}^{d}\right)$ and the map $v \in L_{K, r}^{1}\left(\mathbb{R}^{d}\right) \mapsto \nu * v \in L_{\mathbb{R}}^{\infty}\left(\mathbb{R}^{d}\right)$ is continuous. 
The following Proposition is a consequence of [16] Section 2, Theorem 4 and [23] Theorem 2.23

Proposition 5 1)- For any $\mathcal{L}$ an $r$-discrete subset of $\mathbb{R}^{d}$, let $H_{\mathcal{L}}$ be the Schrödinger operator given by eq. (19). Then, if $v \in L_{K, r}^{1}\left(\mathbb{R}^{d}\right)$ the map $\mathcal{L} \in U D_{r}\left(\mathbb{R}^{d}\right) \mapsto H_{\mathcal{L}}$ is strong resolvent continuous.

2)- If $\Omega$ is the Hull of $\mathcal{L}$, the map $\omega \in \Omega \mapsto H_{\omega}$ is strong resolvent continuous and covariant.

As a consequence, thanks to [16] Theorem 6,

Theorem 7 Let $\mathcal{L}$ be a uniformly discrete set in $\mathbb{R}^{d}$ with Hull $\Omega$. Let $\mathcal{A}=C^{*}\left(\Omega \rtimes \mathbb{R}^{d}, B\right)$ be the $C^{*}$-algebra of the corresponding Noncommutative Brillouin zone. Let $\left\{H_{\omega} ; \omega \in \Omega\right\}$ be the strong resolvent continuous and covariant family of Schrödinger operators defined by eq. (19) with $v \in L_{K, r}^{1}\left(\mathbb{R}^{d}\right)$. Then there is a holomorphic family $z \in \mathbb{C} \backslash \mathbb{R} \mapsto R(z) \in \mathcal{A}$ such that

$$
\pi_{\omega}(R(z))=\left(z-H_{\omega}\right)^{-1}, \quad \forall \omega \in \Omega
$$

As a consequence, it can be said that the Schrödinger operator is affiliated to the $C^{*}$ algebra $\mathcal{A}[55,117,118]$ :

Definition 7 A covariant family $\left(A_{\omega}\right)$ of selfadjoint operators is affiliated to $\mathcal{A}$ if, for all $f \in \mathcal{C}_{0}(\mathbb{R})$, the bounded operator $f\left(A_{\omega}\right)$ can be represented as $\pi_{\omega}\left(A_{f}\right)$ for some $A_{f} \in \mathcal{A}$ such that the map $A: f \in \mathcal{C}_{0}(\mathbb{R}) \mapsto A_{f} \in \mathcal{A}$ is a bounded *-morphism.

The resolvent map $R(z)$ is then given by $A_{r_{z}}$ if $r_{z}: s \in \mathbb{R} \mapsto 1 /(z-s) \in \mathbb{C}$ for $z \in \mathbb{C} \backslash \mathbb{R}$. Conversely the resolvent map permits to reconstruct the map $A$ through a contour integral. It has been argued in [16] that the $C^{*}$-algebra $\mathcal{A}$ above can be actually reconstructed from the Schrödinger operator $H_{\mathcal{L}}$ itself.

In Solid State Physics the conduction electrons are commonly described through the so-called tight binding approximation. This is because only those electrons with energy within $O\left(k_{B} T\right)$ from the Fermi level do contribute to the current. There is no need then to consider the full range of energy to describe these electrons. In particular, the Schrödinger operator can be replaced by its restriction to an energy interval of size $O\left(k_{B} T\right)$ around the Fermi level. However this is not practically accessible in most cases. The method to perform such a reduction goes as follows $[13,61,56]$ for atoms with one valence electron. The single atom Schrödinger equation

$$
H_{1}=\frac{1}{2 m}\left(\frac{\hbar}{\imath} \vec{\nabla}-e \vec{A}\right)^{2}+v(X), \quad \text { on } L^{2}\left(\mathbb{R}^{d}\right)
$$


has usually a non empty discrete spectrum, described by quantum numbers. The valence electron occupy a given eigenstate, denoted by $\phi_{v} \in L^{2}\left(\mathbb{R}^{d}\right)$ corresponding to the energy $E_{v}$. If the potential $v$ decays fast enough to zero at infinity, $\phi_{v}$ decays exponentially fast away from the origin. Then an approximate basis of eigenstate of $H_{\omega}$ is provided by the family $\left\{U(y) \phi_{v} ; y \in \mathcal{L}_{\omega}\right\}$ whenever $U$ is the magnetic translation group. Denoting by $P_{\omega}$ the projection on the subspace generated by such a family in $L^{2}\left(\mathbb{R}^{d}\right)$, it is possible to check that $P_{\omega}=\pi_{\omega}(P)$ for some projection $P \in \mathcal{A}$. Thanks to an orthonormalization procedure, the family $U(y) \phi_{v}$ gives rise to an orthonormal basis $\left\{\psi_{y} ; y \in \mathcal{L}_{\omega}\right\}$ indexed by the atomic sites, where each $\psi_{y}$ is exponentially localized near $y$. By using either the Schur complement (or Feshbach) method [51] or a Grushin method [57] there exists an effective Hamiltonian in the form of a function $z \mapsto H_{\text {eff }}(z)$ holomorphic in a neighbourhood of $E_{v}$ with values in covariant matrices over $\mathcal{L}_{\omega}$. Moreover, the spectrum of the original problem is given by the implicit equation $E \in \mathrm{Sp} H \Leftrightarrow E \in \mathrm{Sp} H_{\text {eff }}(E)$. The advantage of this method is that $H_{\text {eff }}(z)$ can be seen as an element of the groupoid $C^{*}$-algebra $\mathcal{B}$ $[13,16]$, with matrix elements decreasing exponentially fast away from the main diagonal. In practice only a finite number of such diagonals are kept to compute the spectrum, giving rise to tight-binding models. Such a method is actually commonly used also in computer software for the purpose of band calculations in crystals. The various ab initio methods of Quantum Chemistry or the functional density calculations (Kohn-Sham method), may lead to an accurate calculation of the parameters involved in such an effective Hamiltonian. It is not the purpose of these notes to explain these methods. However, they lead to effective Hamiltonians described by elements of the groupoid $C^{*}$-algebra $\mathcal{B}$.

Phonons can also be described in a similar way through $\mathcal{B}$ with zero magnetic field. This is because phonons are the harmonic approximation of the atomic motion around their equilibrium positions. If the atom located at $\vec{x} \in \mathcal{L}$ is moving around $\vec{x}$, let $\vec{x}+\vec{u}_{x}(t)$ be its position at time $t$. Since $x$ is an equilibrium position for the atom, the potential energy created by its neigbours increases away from $x$, hence it is expected that $\vec{u}_{x}(t)$ stay small at all time. Moreover, the lowest order expansion of the potential energy around $x$ gives rise to a quadratic potential, namely harmonic forces, tending to force the atom back to its position $x$. Therefore, within this harmonic approximation, the classical equation of motion for the atom has the form

$$
M \frac{d^{2} \vec{u}_{x}}{d t^{2}}=\sum_{y \in \mathcal{L}_{\omega}} \kappa_{\omega}(x, y)\left(\vec{u}_{y}-\vec{u}_{x}\right),
$$

where $M$ is the atomic mass and $\kappa_{\omega}(x, y)$ is the $d \times d$ matrix of spring constants between atoms located at $y$ and $x$. So $\kappa_{\omega}(x, y) \in M_{d}(\mathbb{R})$. Phonons are just the quantized degrees of freedom associated with this classical motion. In practice however, the influence of atoms far apart is negligible so that $\kappa_{\omega}(x, y)$ decays fast enough as $|x-y| \rightarrow \infty$. Moreover, the action-reaction principle implies that $\kappa_{\omega}(x, y)=\kappa_{\omega}(y, x)$. In addition, since it describes an attracting force, $\kappa_{\omega}(x, y)$ is a positive matrix for any $(x, y)$. Also, the translation 
invariance of interactions between atoms leads to the covariance condition

$$
\kappa_{\mathrm{T}^{a} \omega}(x+a, y+a)=\kappa_{\omega}(x, y) .
$$

In very much the same way, the translation invariance of interactions between atoms leads to the continuity of $\kappa_{\omega}(x, y)$ with respect to $\omega$. Hence the map $(\omega, x) \in \Gamma(X) \mapsto$ $\kappa_{\omega}(0, x) \in M_{d}\left(\mathbb{R}^{d}\right)$ defines a positive element $\kappa$ of $C^{*}(\Gamma(x)) \otimes M_{d}$. The equation of motion (20) is usually solved by looking at the stationnary solutions, namely solutions for which

$$
\frac{d^{2} \vec{u}_{x}}{d t^{2}}=-\tilde{\omega}^{2} \vec{u}_{x}
$$

leading to the eigenmode equation

$$
\sum_{y \in \mathcal{L}_{\omega}}\left\langle x \mid \widehat{\pi}_{\omega}(K) y\right\rangle \vec{u}_{y}=\tilde{\omega}^{2} \vec{u}_{x}, \quad K(\omega, x)=\delta_{x, 0} \sum_{y \in \mathcal{L}_{\omega}} \frac{\kappa(\omega, y)}{M}-\left(1-\delta_{x, 0}\right) \frac{\kappa(\omega, x)}{M}
$$

It is easy to check that this $K$ defines a positive element of $\mathcal{B}$ so that the eigenmodes are given by the spectrum of $K^{1 / 2}$. These modes are the plane waves that are allowed to propagate through the crystal. Historically, Einstein [48] was the first to propose a quantized version of these oscillations, assuming that only one mode was propagating. Then he could compute the heat capacity of the solid and show that it saturates at high temperature leading to the Dulong an Petit law. Soon after, Debye ([64] Section 1.1) introduced a distribution of eigenmodes together with a density of eigenmodes, namely the number of eigenmodes per unit volume and unit of frequency at a given value of $\tilde{\omega}$. He also introduced a cut-off to take into account the uniform discreteness of the crystal, in the form of the Debye Temperature $\Theta_{D}$. In 1912, Born and von Karman [28] performed the first explicit calculation of the eigenmodes in a cubic crystal and discovered the existence of optical modes, explaining a small discrepancy between the Debye predictions and the measurements of the heat capacity.

In both cases, the density of states (DOS), for electrons, and the vibrational density of states (VDOS), for the phonons, are defined in a similar way. In the phonon case, the relevant operator is $K^{1 / 2} \in \mathcal{B}$, whereas for electrons it is its Hamiltonian $H$, namely either the Schrödinger operator, in the continuum case, or the effective Hamiltonian in the tight binding representation. Let then $H$ be the name of such an operator. In both cases it is given by a strong resolvent continuous family $\left(H_{\omega}\right)_{\omega}$ of selfadjoint operators, bounded from below, indexed either by the Hull $\Omega$ or by the transversal $X$. It acts either on $L^{2}\left(\mathbb{R}^{d}\right)$ or on $\ell^{2}\left(\mathcal{L}_{\omega}\right)$. In both cases, given a bounded box $\Lambda \subset \mathbb{R}^{d}$, it is meaningfull to restrict $H_{\omega}$ to $\Lambda$ provided boundary conditions are prescribed. Let $H_{\omega, \Lambda}$ be this restriction. Then its spectrum is discrete in both cases so that there is only a finite number of eigenvalues (counted with their multiplicities), below $E \in \mathbb{R}$. This allows to define the integrated density of states (IDS) as 


$$
\mathcal{N}(E)=\lim _{\Lambda \uparrow \mathbb{R}^{d}} \frac{1}{|\Lambda|} \#\left\{E^{\prime} \in \operatorname{Sp}\left(H_{\omega, \Lambda}\right) ; E^{\prime} \leq E\right\}
$$

The existence of the limit depends upon the choice of an invariant ergodic probability measure $\mathbb{P}$ on the Hull so that the limit exists only $\mathbb{P}$-almost surely (resp. $\mathbb{P}_{t r}$-almost surely) with respect to $\omega[13,33,43,86]$. Let then $\mathcal{N}_{\mathbb{P}}$ denote this common limit. The Shubin formula $[13,16]$ expresses it in term of the noncommutative Brillouin zone as

$$
\mathcal{N}_{\mathbb{P}}(E)=\mathcal{T}_{\mathbb{P}}(\chi\{H \leq E\}), \quad \text { (Shubin formula) }
$$

with a similar formular in the tight-binding case. Here $\chi\{\cdot \leq E\}$ denotes the characteristic function of the interval $(-\infty, E] \subset \mathbb{R}$. Then $\chi\{H \leq E\}$ is the spectral projection of $H$ seen as an element of the von Neumann algebra $L^{\infty}\left(\mathcal{A}, \mathcal{T}_{\mathbb{P}}\right)$. It is then clear from the definition, that $\mathcal{N}_{\mathbb{P}}$ is a non decresing non negative function of $E$. It vanishes below $E_{0} \inf \operatorname{Sp}\{H\}$. It is constant on spectral gaps (see Fig. 5). In particular, its derivative $d \mathcal{N}_{\mathbb{P}} / d E$ exists in the sense of a Stieljes-Lebesgue measure and is called the density of states (DOS) (resp. the vibrational density of states).

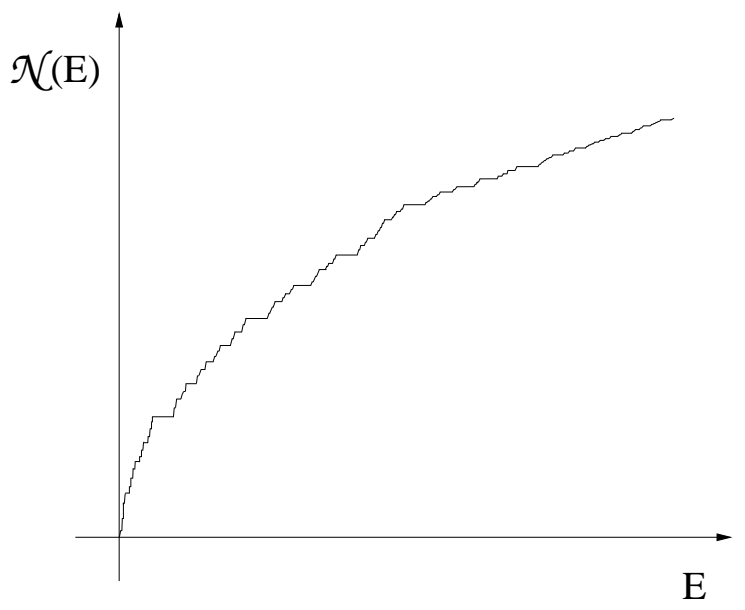

Figure 5: An example of electronic integrated density of states in 1D

The DOS is usually used to expressed thermodynamical quantities such as the heat capacity. In the case of phonons, the VDOS is given by

$$
\mathcal{N}_{\phi}(\tilde{\omega})=\hat{\mathcal{T}}_{\mathbb{P}}\left(\chi\left(K \leq \tilde{\omega}^{2}\right)\right) \rho_{\mathbb{P}}, \quad \text { (Shubin for phonons) }
$$

where $\rho_{\mathbb{P}}$ is the atomic density (in number of atoms per unit volume) in order to get a number of modes per unit volume. Because there are $d$ possible choices for the polarization of the acoustic waves, the trace $\hat{\mathcal{T}}_{\mathbb{P}}(\chi(K \leq \infty))=d$. The Debye formula for the phonon contribution to heat capacity per unit volume leads to 


$$
C_{v}^{p h}=k_{B} \int_{0}^{\tilde{\omega}_{D}} d \mathcal{N}_{\phi}(\tilde{\omega})\left\{\frac{\hbar \tilde{\omega} / 2 k_{B} T}{\sinh \left(\hbar \tilde{\omega} / 2 k_{B} T\right)}\right\},
$$

where $\tilde{\omega}_{D}$ is the Debye cut-off frequency of the solid. If $T \uparrow \infty$, eq. (24) gives the DulongPetit law, namely

$$
C_{V}^{p h} \stackrel{T \uparrow \infty}{\longrightarrow} \rho_{\mathbb{P}} k_{B} d, \quad \text { (Dulong \& Petit). }
$$

As $T \downarrow 0$, only the low frequencies contribute, so that it is enough to consider the asymptotic of the VDOS at $\tilde{\omega} \downarrow 0$. Since low frequencies correspond to large wave lengths, the corresponding phonons do not see the fine structure of the solid and behave like acoustic waves with sound velocity $c_{\alpha}$ for the polarisation $\alpha$. In particular $d \mathcal{N}_{\phi}(\tilde{\omega})$ behaves like $\tilde{\omega}^{d-1} d \tilde{\omega}$, so that

$$
C_{v}^{p h} \propto T^{d}, \quad \text { as } T \downarrow 0 .
$$

For $d=3$ this gives the usual $T^{3}$ law discovered by Debye.

The electronic contribution to the heat capacity must take the fermionic character of the electrons into account. Then

$$
C_{v}^{e l}=T\left(\frac{\partial S}{\partial T}\right)_{V, N}
$$

if $S$ is the total entropy in the volume $V$. The derivative is taken at constant volume and constant electron number. The computation of $S$ is usually performed in the grand canonical ensemble. If $\mathcal{N}_{e l}$ denotes the electronic IDS, the chemical potential $\mu$ is fixed by the condition

$$
2 \int_{\mathbb{R}} d \mathcal{N}_{e l}(E) \frac{1}{1+e^{-\beta(E-\mu)}}=\frac{N}{V}=2 \mathcal{N}_{e l}\left(E_{F}\right) .
$$

Here $N$ is the total number of electrons in volume $V$ and $E_{F}$ is called the Fermi energy. The factor 2 comes from the spin degrees of freedom. Then the entropy $S$ is given by

$$
S=2 k_{B} V \int_{\mathbb{R}} d E \beta^{2}(E-\mu) \frac{\mathcal{N}_{e l}(E)}{4 \cosh ^{2}\{\beta(E-\mu) / 2\}} .
$$

Here $\beta=1 / k_{B} T$. Setting $x=\beta(E-\mu) / 2$ this gives

$$
S=2 k_{B} V \int_{0}^{\infty} d x \frac{x}{\cosh ^{2}(x)}\left(\mathcal{N}_{e l}\left(\mu+2 x k_{B} T\right)-\mathcal{N}_{e l}\left(\mu-2 x k_{B} T\right)\right) .
$$

Whenever the IDS is smooth around $E=E_{F}$, this gives the usual electronic contribution to the heat capacity

$$
C_{v}^{e l} \propto T, \quad \text { as } T \downarrow 0
$$


If, however $\mathcal{N}_{e l}$ admits some fractal behaviour near the Fermi energy, then the low temperature behaviour may be modified. It turns out that such a fractal behaviour is hardly seen in practice. This is because the disorder and the interactions as well tend to smooth out the IDS at small energy scales.

\section{Examples of Hull}

\subsection{Perfect Crystals}

If $\mathcal{L}$ denotes the set of atoms of a perfect crystal, let $\mathbb{G}$ be its period group (see Section 2.5). Then clearly the set of translated of $\mathcal{L}$ can be identified with the quotient space $\mathbb{R}^{d} / \mathbb{G}=\mathbb{V}$ by the very definition of the period group. In particular, $\mathbb{G}$ is cocompact. Thus the Hull is the Wigner-Seitz cell with periodic boundary conditions. Since any cocompact subgroup of $\mathbb{R}^{d}$ is isomorphic to $\mathbb{Z}^{d}$, it follows that $\Omega$ is homeomorphic to a $d$-torus. In very much the same way, the transversal is the finite set $X=\mathcal{L} / \mathbb{G}$. The number of points in $X$ is the number of atoms in each unit cell. The groupoid of the transversal $\Gamma(X)$ is made of pairs $(x, b) \in X \times \mathbb{R}^{d}$ such that $(x-b) \bmod \mathbb{G} \in X$. Such $b$ 's can be labelled in the following way. If $x-b=y \bmod \mathbb{G}$ an element of $\Gamma(X)$ can be seen as a triplet $(x, y ; a) \in X \times X \times \mathbb{G}$ with the range, source maps and composition given by

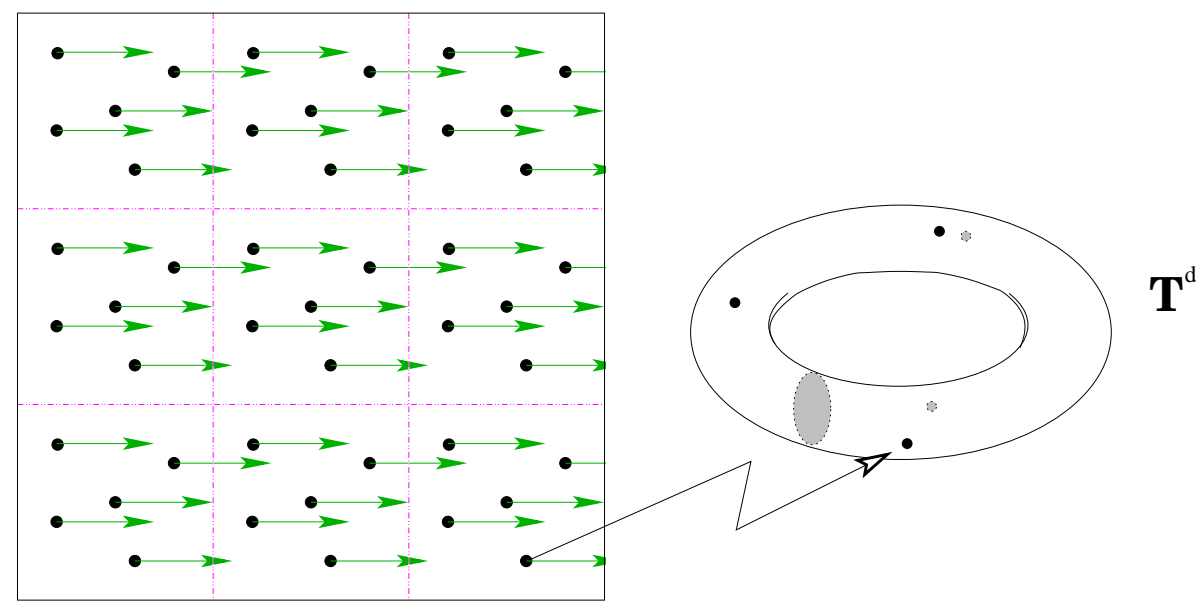

Figure 6: The Hull of a periodic crystal

$$
r(x, y ; a)=x \quad s(x, y ; a)=y, \quad(x, y ; a) \circ(y, z ; b)=(x, z ; a+b)
$$

In particular, a typical element of the $C^{*}$-algebra associated with this groupoid is a matrix valued sequence $F_{x, y}(a)$ with product and adjoint given by 


$$
(F G)_{x, y}(a)=\sum_{z \in X} \sum_{b \in \mathbb{G}} F_{x, z}(b) G_{z, y}(a-b), \quad\left(F^{*}\right)_{x, y}(a)=\overline{F_{y, x}(-a)} .
$$

In particular, using the Fourier transform (here $\mathbb{B}$ is the dual group to $\mathbb{G}$ and has been called the Brillouin zone in Section 2.5)

$$
(\widehat{F}(k))_{x, y}=\sum_{a \in \mathbb{G}} F_{x, y}(a) e^{\imath\langle k \mid a\rangle}, \quad k \in \mathbb{B}
$$

the previous algebra becomes nothing else but the tensor product $\mathcal{C}(\mathbb{B}) \otimes M_{n}(\mathbb{C})$ if $n=|X|$ is the number of points contained in $X$. The same argument shows that the $C^{*}$-algebra of the Hull is isomorphic to $\mathcal{C}(\mathbb{B}) \otimes \mathcal{K}$ where $\mathcal{K}$ is the $C^{*}$-algebra of compact operators.

\subsection{Disordered Systems}

Let us consider now the case of impurities in a perfect monoatomic crystal. The physical example is the silicon diamond lattice in which some of the $S i$ atoms may be replaced by an impurity of the columns IIIA ( $B, A l, G a, I n$ or $p$-type), or VA ( $P, A s, S b$ or $n$-type). The finite set of possible doping atoms is denoted by $\mathfrak{A}$ and will be called an alphabet. For example, if the $S i$ is doped only with boron $B$ or antimony $S b$, the alphabet will contain three letters, namely $\mathfrak{A}=\{S i, B, S b\}$. Let $\mathcal{L}$ be the underlying lattice of the pure silicon. Then, if $x \in \mathcal{L}$ let $n_{x} \in \mathfrak{A}$ be the letter denoting the atom sitting at $x$. A family of the type $\underline{n}=\left(n_{x}\right)_{x \in \mathcal{L}} \in \mathfrak{A}^{\mathcal{L}}$ will be called a configuration of impurities. The period group $\mathbb{G}$ of $\mathcal{L}$ acts on the configuration space $\mathfrak{A}^{\mathcal{L}}$ through

$$
\mathrm{T}^{a} \underline{n}=\left(n_{x-a}\right)_{x \in \mathcal{L}}, \quad a \in \mathbb{G}
$$

To take into account that most of the lattice sites are occupied by a silicon atom, it is necessary to define a probability measure $\mathbb{P}$ which forces the impurities to be very rare. The simplest example of such a probability consists in demanding that the $n_{x}$ 's be independent identically distributed random variables with a common distribution $p$ such that $p(S i)=1-c$ where $c=\sum_{w \in \mathfrak{A} \backslash\{S i\}} p(w) \in(0,1)$ is the concentration of impurities. Then $\mathbb{P}(\underline{n})=\bigotimes_{x \in \mathcal{L}} p\left(n_{x}\right)$ is $\mathbb{G}$-invariant. The main result is the following $[105,23]$

Proposition 6 If $p(w)>0$ for all letter $w \in \mathfrak{A}$, namely if each impurity occurs with a nonzero concentration, then

(i) for $\mathbb{P}$-almost all configuration $\underline{n}$ of impurities, the transversal $X$ of the Hull of $\underline{n}$ is homeomorphic to $\mathfrak{A}^{\mathcal{L}}$. In particular $X$ is a Cantor set.

(ii) the Hull is homeomorphic to the suspension of $X$ by the action of $\mathbb{G}$, namely

$$
\Omega \simeq X \times \mathbb{R}^{d} / \mathbb{G}
$$

where $\mathbb{G}$ acts on $X$ through $\mathrm{T}$ and on $\mathbb{R}^{d}$ by translation. 
The previous result has been generalized to probabilities $\mathbb{P}$ with correlations in [105, 23]. The condition that each impurity occurs with a nonzero concentration must be then replaced by: given any finite subset $\Lambda \subset \mathcal{L}$ then any configuration $n_{\Lambda}=\left(n_{x}\right)_{x \in \Lambda}$ in $\mathfrak{A}^{\Lambda}$ have a non zero probability. Such a condition is usually satisfied by Gibbs measures describing the thermal equilibrium of the impurities.

\subsection{Quasicrystals}

The simplest example of non-periodic tiling was provided by R. Penrose [90] (see Fig. 7). It is built from two types of tiles in the 2D plane, through inflation rules, and exhibits a five-fold symmetry. It was extensively studied by de Bruijn [31]. But it was recognized only later on by physicists that it is quasiperiodic. However, de Bruijn and also Kramer \& Neri [74] built examples of quasiperiodic tilings. Most models describing the quasiperiodic order in quasicrystals, are based upon the so-called cut-and-project method, independently proposed by Duneau \& Katz [46, 47], Kalugin, Kitaev \& Levitov [67], Elser [49] and Levine \& Steinhardt [81]. It was not until 1995 that this method was recognized as equivalent to the notion of model sets provided by Meyer [83] in his thesis work [82].

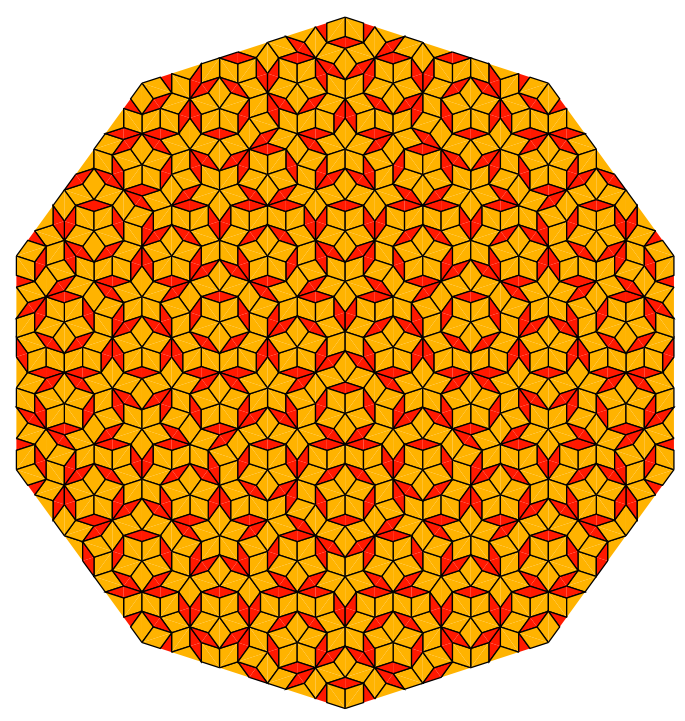

Figure 7: The Penrose tiling

The best models known nowdays to represent the structure of a quasicrystal are built by using the cut-and-project method (see [62]). It is well illustrated by the construction given in Fig. 8. The idea is the following. Let $N=d+n$ be an integer bigger than the dimension of the physical space. In $\mathbb{R}^{N}$, the lattice $L=\mathbb{Z}^{N}$ defines some periodicity. Then let $E_{\|}$be a $d$-dimensional subspace of $\mathbb{R}^{N}$ intersecting $L$ only at the origin (irrationality condition). The idea of the construction is that the physical space is precisely $E_{\|}$. Then $E_{\perp}$ will denote the orthocomplement subspace. The corresponding orthogonal projection on $E_{\|}$ 


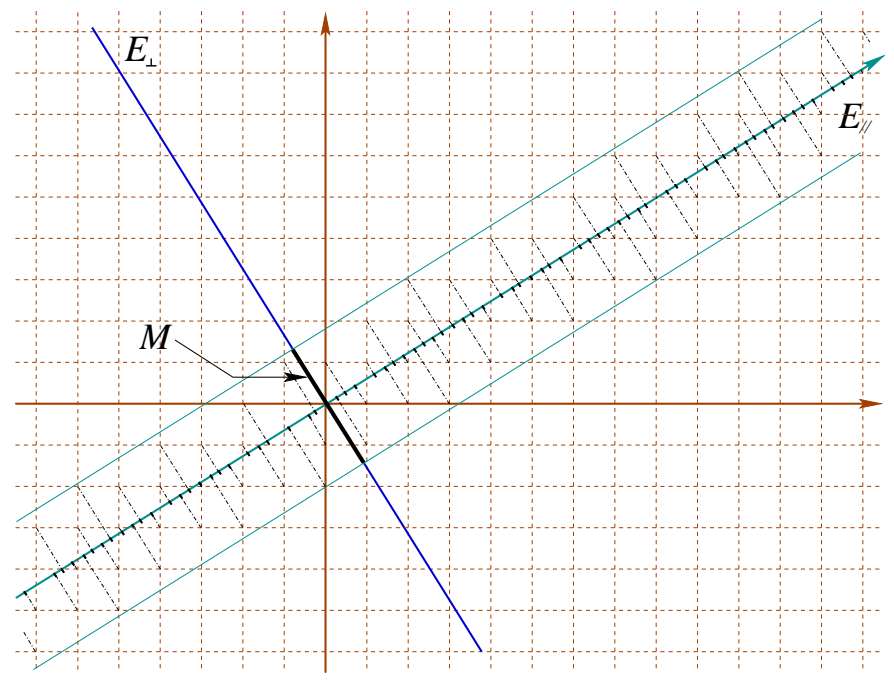

Figure 8: The cut-and-project construction

and $E_{\perp}$ will be denoted by $\pi_{\|}, \pi_{\perp}$ respectively. Let then $W$ be the polytope obtained by projecting the unit $N$-cube on $E_{\perp}$ and let $O$ be its center, namely the projection of the center of the $N$-cube. $W$ will be called the window or the acceptance domain. Given a face of $W$ of maximal dimension $n-1$, there is a unique opposite face obtained by the inversion symmetry around $O$. For each such a pair of faces, one, called permitted, will be added to $W$ the other one, called forbidden, will be excluded from $W$. If two $(n-1)$-permitted faces are adjacent, the closed $(n-2)$-face they have in common will be taken in $W$. Otherwise the same procedure must be applied to $(n-2)$-faces, then to the $(n-3)$-faces etc. In this way, for each pair of opposite points of the boundary of $W$ one is permitted, and belongs to $W$, the other one is forbidden. Thus, $W$ is neither closed nor open. Let then $\Sigma_{W}=W+E_{\|}$be the strip parallel to the physical space and generated by $W$. The lattice points contained in $\Sigma_{W}$, are then projected on the physical space to give the atomic sites $\mathcal{L}$, namely (see Fig. 8)

$$
\mathcal{L}=\pi_{\|}\left(\left(W+E_{\|}\right) \cap \mathbb{Z}^{d}\right)
$$

Example 4 (The octagonal tiling-) The simplest example of such a construction is the octagonal or Ammann-Beenker tiling given in Fig. 9

To built this model it is enough to start from $\mathbb{Z}^{4}$. The 8 -fold symmetry seen in Fig. 9 can be implemented through the transformation (see Fig. 10) 


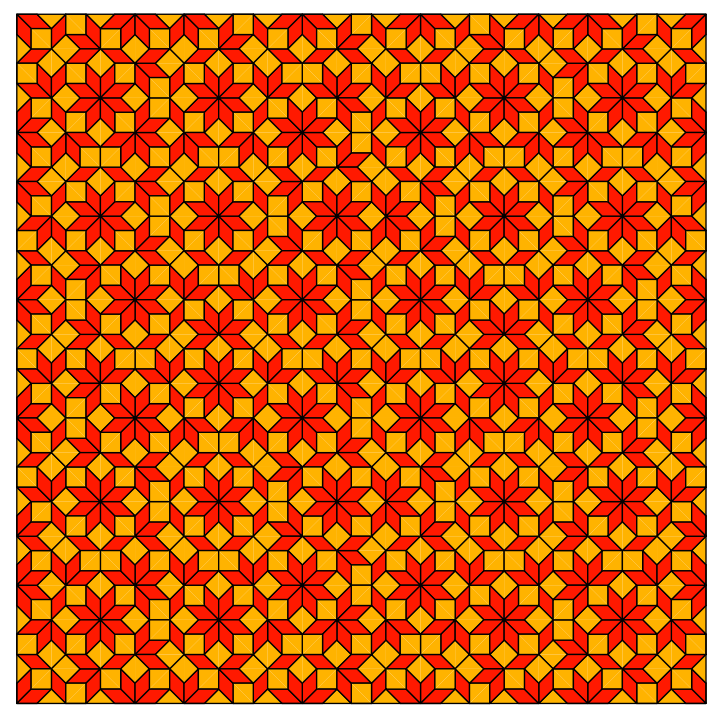

Figure 9: The Ammann-Beenker tiling

$$
R:\left\{\begin{array}{c}
e_{1} \mapsto e_{3} \\
e_{2} \mapsto e_{4} \\
e_{3} \mapsto e_{2} \\
e_{4} \mapsto-e_{1}
\end{array}\right\}
$$

where $\left\{e_{1}, e_{2}, e_{3}, e_{4}\right\}$ is the canonical basis of $\mathbb{R}^{4}$. The matrix $R$ has integer coefficients, is orthogonal and satisfies $R^{4}=-1$. It can be decomposed into the direct sum of two plane rotations of angles $\pi / 4$ and $3 \pi / 4$. The corresponding invariant planes will be $E_{\|}$and $E_{\perp}$ respectively. Since both these planes and the lattice $\mathbb{Z}^{4}$ are $R$-invariant, and because $R$ commutes with the projections $\pi_{\|}, \pi_{\perp}$, it follows that $R$ leaves the set $\mathcal{L}$ of atomic sites invariant, which is what is seen in Fig. 9. The acceptance domain is the projection of the unit 4-cube on $E_{\perp}$. It must be $R$ invariant, so that it must be an octagon.

This acceptance domain is given in Fig. 10 together with the projection of the four basis vectors in $E_{\perp}$. The acceptance domain can be used to read the properties of the tiling. For instance, le $\mathcal{L}_{3}$ the set of points in $\mathcal{L}$ having exactly three nearest neighbours in the direction $e_{1}, e_{3},-e_{4}$. Such points come from points $a \in L$ such that $a+e_{1}, a-e_{4}, a+e_{3}$ belong to the strip $\Sigma_{W}$ whereas the other $a \pm e_{i}$ 's fall out of $\Sigma_{W}$. Their projection onto $W$ gives the subdomain of $W$ given by the rectangle triangle with hypothenuse on the boundary of $W$ and located along the direction $e_{1}$ from the center. The other subdomains designed in Fig. 10 correspond to the acceptance domains of sites with other possible nearest neighbour environments.

The transversal of $\mathcal{L}$ is nothing but the acceptance domain $W$ provided it is completed for a topology finer than the usual topology in $\mathbb{R}^{2}$. More precisely, given any finite family 

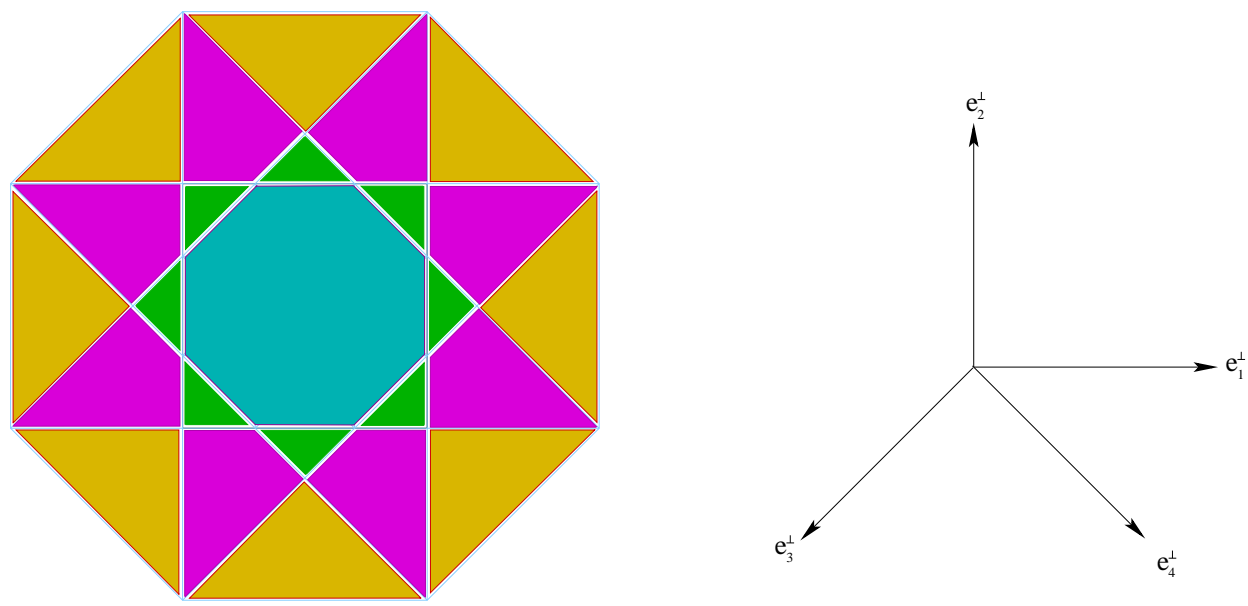

Figure 10: The acceptance domain of the Ammann-Beenker tiling

of vectors $\left(a_{1}, \cdots, a_{l}\right)$ in $\mathbb{Z}^{4}$, the sets $W \cap\left(W+\pi_{\perp}\left(a_{1}\right)\right) \cap \cdots \cap\left(W+\pi_{\perp}\left(a_{l}\right)\right)$ are closed and open for this new topology. In particular all possible acceptance domain of local environments are closed and open. It is easy to see that $W$ is a Cantor set.

To built the Hull, it is enough to pull-back the topology of $W$ to $\mathbb{R}^{4}$ by demanding that $\pi_{\perp}^{-1}\left(W_{0}\right)$ be closed and open for each $W_{0} \subset W$ closed and open. Let $\mathbb{R}_{W}^{4}$ denote the completion of $\mathbb{R}^{4}$ for this topology. It is a locally compact space on which both $\mathbb{Z}^{4}$ and $E_{\|}$acts by homeomorphisms. In particular the Hull can be identified with $\mathbb{T}_{W}^{4}=\mathbb{R}_{W}^{4} / \mathbb{Z}^{4}$ and $\mathbb{R}^{2}$ acts by translation by vectors of $E_{\|}$.

In general the cut-and-project method can be well described in term of model sets of Meyer. Namely it is defined with spaces and maps as follows

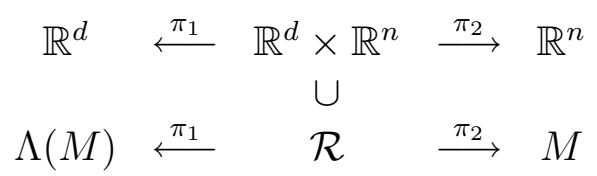

where $\mathcal{R} \subset \mathbb{R}^{d} \times \mathbb{R}^{n}$ is a lattice (a co-compact discrete subgroup) and $\pi_{1}$ and $\pi_{2}$ are the projections onto $\mathbb{R}^{d}$ and $\mathbb{R}^{n}$, respectively. Furthermore $\pi_{1}$ restricted on $\mathcal{R}$ is injective and $\pi_{2}(\mathcal{R})$ is dense in $\mathbb{R}^{n} . \mathbb{R}^{d}$ wil be called the physical space and $\mathbb{R}^{n}$ the internal space. Here $\pi_{1}$ and $\pi_{2}$ are the restriction maps on the corresponding coordinate of $\mathbb{R}^{d} \times \mathbb{R}^{n}$. Therefore the setting of a cut-and-project scheme is given by the triple $\left(\pi_{1}, \pi_{2}, \mathcal{R}\right)$. For a subset $M$ in the internal space $\mathbb{R}^{n}$ we define the corresponding point set in the physical space $\mathbb{R}^{d}$ as

$$
\Lambda(M)=\left\{\pi_{1}(a) \mid a \in \mathcal{R}, \pi_{2}(a) \in M\right\} .
$$

$M$ is called the acceptance domain of the point set $\Lambda(M)$. For a lattice vector $a \in \mathcal{R}$

$$
\Lambda\left(M+\pi_{2}(a)\right)=\Lambda(M)+\pi_{1}(a)
$$


Definition 8 A point set $L$ in $\mathbb{R}^{d}$ is called a model set if there exists a bounded set $M$ with non-empty interior such that $L=\Lambda(M)$.

The next result is proved in [23]

Proposition 7 A model set is a Meyer set.

Definition 9 Let $M$ be a bounded subset of $\mathbb{R}^{n}$ with non-empty interior. $M$ is called admissible if for every ball $B(x, \epsilon)$ with $\epsilon>0$ and $x$ in $\pi_{2}(\mathcal{R}) \cap M$, there exists a finite family $\left\{a_{1}, \ldots, a_{p}\right\}$ in $\pi_{2}(\mathcal{R})$ such that $M \cap\left(M+a_{1}\right) \cap \ldots \cap\left(M+a_{p}\right)$ is a subset of $B(x, \epsilon)$ with non-empty interior. A model set $L$ is called admissible if there exists an admissible set $M$ such that $L=\Lambda(M)$.

Example 5 A convex polytope is an admissible set.

Let $L=\Lambda(M)$ be an admissible model set and let $\mathcal{A}_{M}$ denote the $C^{*}$-algebra generated by the set of functions $\left\{f \otimes\left(\chi_{M} \circ T_{n}^{\pi_{2}(a)}\right) ; a \in \mathcal{R}, f \in \mathcal{C}_{c}\left(\mathbb{R}^{d}\right)\right\}$, where $T_{n}^{\pi_{2}(a)}$ denotes the translation in $\mathbb{R}^{n}$ by $\pi_{2}(a)$. Here $\chi_{M}$ denotes the characteristic function of $M$. Let $\mathbb{R}_{M}^{n+d}$ be the set of characters of $\mathcal{A}_{M}$ so that, by Gelfand's theorem, $\mathcal{A}_{M}$ is isomorphic to $\mathcal{C}_{0}\left(\mathbb{R}_{M}^{n+d}\right)$ where $\mathbb{R}_{M}^{n+d}$ is a locally compact space. Since $M$ is admissible $\mathcal{C}_{0}\left(\mathbb{R}^{n+d}\right)$ is a closed subalgebra of $\mathcal{A}_{M}$. By duality there is a surjective continuous map $\pi_{M}: \mathbb{R}_{M}^{n+d} \rightarrow \mathbb{R}^{n+d}$. Therefore $\mathbb{R}_{M}^{n+d}$ can be seen as the completion of $\mathbb{R}^{n+d}$ for a finer topology than the usual one, that will be called the $M$-topology, in which the sets $\mathbb{R}^{d} \times M+a$, for $a \in \mathcal{R}$, are open and closed. By construction, for $a \in \mathcal{R}$, the map $x \in \mathbb{R}^{d} \times \mathbb{R}^{n} \rightarrow x+a \in \mathbb{R}^{d} \times \mathbb{R}^{n}$ extends to $\mathbb{R}_{M}^{n+d}$ by continuity. One sets $\mathbb{T}_{M}^{n+d}=\mathbb{R}_{M}^{n+d} / \mathcal{R}$. By construction, for $y \in \mathbb{R}^{d} \times\{0\}$, the map $x \in \mathbb{R}^{d} \times \mathbb{R}^{n} \rightarrow x+y \in \mathbb{R}^{d} \times \mathbb{R}^{n}$ extends also by continuity to $\mathbb{R}_{M}^{n+d}$ and commutes with the action of $\mathcal{R}$. Thus it defines a $\mathbb{R}^{d}$-action $\hat{T}$ on $\mathbb{T}_{M}^{n+d}$. Similarly $\mathbb{R}_{M}^{n}$ can be defined as the set of characters of the $C^{*}$-algebra generated by the set $\left\{\chi_{M} \circ T_{n}^{\pi_{2}(a)} ; a \in \mathcal{R}\right\}$. Since it is a set of idempotents, $\mathbb{R}_{M}^{n}$ is totally disconnected and $\mathbb{T}_{M}^{n+d}$ is transversally totally disconnected.

Definition 10 The dynamical system $\left(\mathbb{T}_{M}^{n+d}, \mathbb{R}^{d}\right)$ is called the pseudo-torus associated with the window $M$.

The following result is proved in [23]

Theorem 8 Let $L=\Lambda(M)$ be an admissible model set in $\mathbb{R}^{d}$, and $\nu=\nu^{(L)}$. Then $\left(\Omega_{\nu}, \mathbb{R}^{d}, T\right)$ is topologically conjugated to $\left(\mathbb{T}_{M}^{n+d}, \mathbb{R}^{d}, \hat{T}\right)$. This dynamical system is minimal. It is uniquely ergodic, providing $M$ is a Borel set in $\mathbb{R}^{n}$.

Up to now, $M$ has only been assumed to be admissible. The following property actually holds in quasicrystals: 
Definition 11 A polytope in $\mathbb{R}^{n}$ is said $\mathcal{R}$-compatible if its vertices belong to $\pi_{2}(\mathcal{R})$.

If the acceptance domain $M$ is an $\mathcal{R}$-compatible polytope, let $F_{1}, \cdots, F_{p}$ be the hyperplanes of $\mathbb{R}^{n+d}$ parallel to the maximal faces of $\mathbb{R}^{n} \times M$. For each $j \in\{1, \cdots, p\}$, let $u_{j} \in \mathbb{R}^{n+d}$ be a unit vector perpendicular to $F_{j}$ so as to define $F_{j}^{+}=\left\{x \in \mathbb{R}^{n+d} ;\left\langle u_{j} \mid x\right\rangle \geq\right.$ $0\}$ and $F_{j}^{-}=\mathbb{R}^{n+d} \backslash F_{j}^{+}$. Let then $\mathcal{F}$ be the family of affine hyperplanes $F_{j}+a$ with $j \in\{1, \cdots, p\}$ and $a \in \pi_{2}(\mathcal{R}) . \mathbb{R}^{d} \times \mathbb{R}^{n}$ is then endowed with the coarsest topology for which, given any $F \in \mathcal{F}$, the closed half-space $F^{+}$is both closed and open. It will be called the $\mathcal{F}$-topology. The same construction can be performed in $\mathbb{R}^{n}$. Let $\mathbb{R}_{\mathcal{F}}^{n+d}$ and $\mathbb{R}_{\mathcal{F}}^{n}$ be the completions of $\mathbb{R}^{d} \times \mathbb{R}^{n}$ and $\mathbb{R}^{n}$ with this topology, respectively. In much the same way, $\mathbb{T}_{\mathcal{F}}^{n+d}=\mathbb{R}_{\mathcal{F}}^{n+d} / \mathcal{R}$ is well defined and can be endowed with an $\mathbb{R}^{d}$-action. The following proposition is easy to prove:

Proposition 8 If $M$ is $\mathcal{R}$-compatible, the $M$-topology and the $\mathcal{F}$-topology are equivalent on $\mathbb{R}^{n+d}$. In particular $\mathbb{T}_{\mathcal{F}}^{n+d}=\mathbb{T}_{M}^{n+d}$.

Remark 7 An alternative description of this pseudo-torus is proposed in $[80,54]$ under very general hypothesis on $M$.

Remark 8 In a Meyer set obtained through the cut-and-project method any bounded pattern repeats itself infinitely often. More generally, each Meyer set is a Delone set of finite type. Thanks to Proposition 4, this implies that the canonical transversal is totally disconnected. This can also be seen from the particular topology described just before.

\section{$3.4 \quad$ Finite type Tilings}

The construction of quasicrystalline lattices given in the previous Section 3.3 cannot be extended to any Delone set of finite type. Nevertheless a construction has been proposed in [24], inspired by earlier papers by $[4,103]$, which gives the description of the Hull in such a case. The consequence is summarized in the Theorem 2 given at the end of Section 2.3. Let this theorem be illustrated with the help of the octagonal tiling.

The octagonal tiling shown in Fig 9 can be tiled by 20 tiles modulo translation. Each tile is repeated eight times when the 8-fold rotation $R$ is applied (see Section 2.3). Therefore, modulo $R$, three tiles are remaining, one is a rhombus with sides of length one and a $45^{\circ}$ angle, the two others are rectangle triangles corresponding to half a square of side one. The two half squares are symmetric to each other around the diagonal of the square. These tiles are also decorated by arrows. They are shown in Fig. 11 below. The arrows have to match within the tiling. The octagonal tiling is invariant by an inflation symmetry of ratio $\sqrt{2}+1$. The inflated tiles are also shown in Fig. 11. For instance, the inflated rhombus is made of three rhombi and two of each variety of half squares. The inflated square is made of 4 rhombi and 3 of each variety of half squares. 


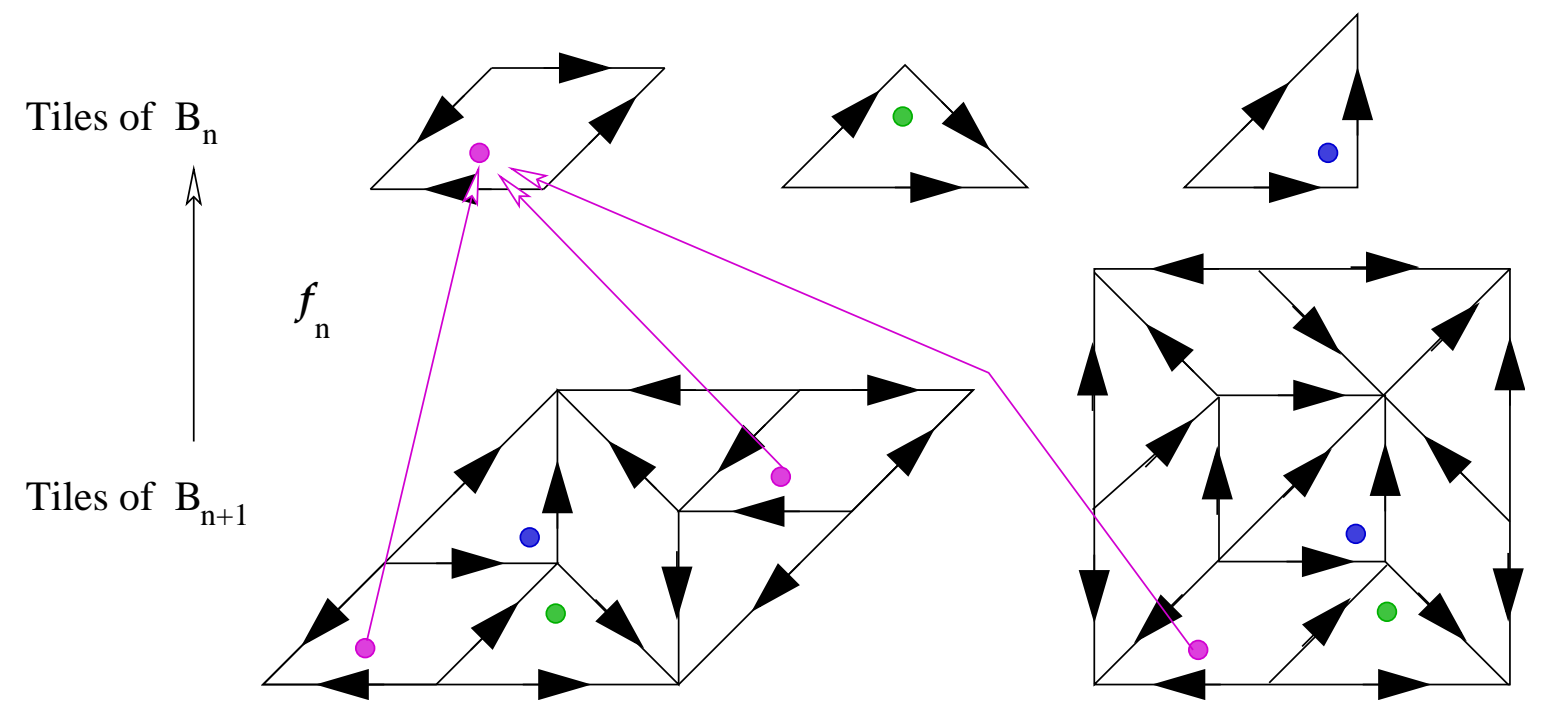

Figure 11: Construction of BOF manifolds for the octagonal tiling

The family of these 20 tiles is made into a compact branched, oriented, flat Riemannian manifold (BOF) by identifying the oriented sides of various tiles whenever they match somewhere in the tiling. The result is the manifold $B_{0}$. To obtain $B_{1}$, it is sufficient to proceed the same way with the inflated tiles. The decoration of the sides of the inflated tiles is now more complicated. But the same rule applies. Due to the inflation symmetry, $B_{0}$ and $B_{1}$ are actually diffeomorphic BOF manifolds. However, the inflation changes the metric from $d s^{2}$ to $(\sqrt{2}+1)^{2} d s^{2}$. It is thus possible to define a map $f_{0}: B_{1} \mapsto B_{0}$, with derivative $D f_{0}=\mathbf{1}$ (the unit matrix), which identifies each tile of order 0 in an inflated tile with the corresponding tile of $B_{0}$ (see Fig. 11). It is important to remember that two tiles which differ by a rotation are considered as distinct. Such a map is called a BOF submersion. It is locally the identity map, it is globally onto, but it maps several point of $B_{1}$ to the same point in $B_{0}$. In a sense, $f_{0}$ folds $B_{1}$ onto $B_{0}$.

Then, by inflating $n$ times, and proceeding in the same way at each inflation, the BOF manifold $B_{n}$ is constructed. Again, in this particular example, due to the inflation symmetry, $B_{n}$ is diffeomorphic to $B_{0}$. But the metric $d s^{2}$ is multiplied by $(\sqrt{2}+1)^{2 n}$ in $B_{n}$. In very much the same way, as shown in Fig. 11, there is a BOF submersion $f_{n}: B_{n+1} \mapsto B_{n}$ defined by identifying the $n$-tiles tiling the $(n+1)$-tiles in $B_{n+1}$ with the corresponding $n$-tile of $B_{n}$.

This construction leads to a projective family $\cdots \stackrel{f_{n+1}}{\longrightarrow} B_{n+1} \stackrel{f_{n}}{\longrightarrow} B_{n} \stackrel{f_{n-1}}{\longrightarrow} \cdots$ of BOF manifolds. Then the successive unfolding of the $B_{n}$ 's is described through the projective limit

$$
\Omega=\lim _{\leftarrow}\left(B_{n}, f_{n}\right)
$$


By definition, a point in $\Omega$ is a family $\omega=\left(x_{n}\right)_{n \in \mathbb{N}} \in \prod B_{n}$ such that $f_{n}\left(x_{n+1}\right)=x_{n}$ for all $n$ 's. Actually only the large enough $n$ 's do matter to define $\omega$, since given $x_{n}$, all the $x_{j}$ with $j<n$ are uniquely defined. Therefore, given $a \in \mathbb{R}^{2}$, there is $N$ large enough so that $x_{n}$ is at distance larger than $\|a\|$ from the boundary of the tile it belongs to. In particular, $x_{n}+a$ is a well defined point in the same tile as $x_{n}$. Since $D F_{n}=\mathbf{1}$, it follows that $f_{n}\left(x_{n+1}+a\right)=x_{n}+a$ for $n$ large enough. Thus the point $\mathrm{T}^{a} \omega=\left(y_{n}\right)_{n \in \mathbb{N}}$ defined by $y_{n}=x_{n}+a$ for $n>N$ is well defined in $\Omega$. Hence $\mathbb{R}^{2}$ acts on $\Omega$ through $\mathrm{T}$. The Theorem 2 says that the dynamical system $\left(\Omega, \mathbb{R}^{2}, \mathrm{~T}\right)$ is conjugate to the Hull of the tiling through a homeomorphism.

In the general situation of a Delone set $\mathcal{L} \subset \mathbb{R}^{d}$ that is repetitive with finite type, the procedure is similar. First, a tiling is built, by means of the Voronoi construction. Namely given $x \in \mathcal{L}$, the Voronoi cell of $x$ is the open convex polyhedron $V_{x}$ defined as (see Fig. 12)

$$
V_{x}=\left\{z \in \mathbb{R}^{d} ;\|z-x\|<\|z-y\|, \forall y \in \mathcal{L} \backslash\{x\}\right\}
$$
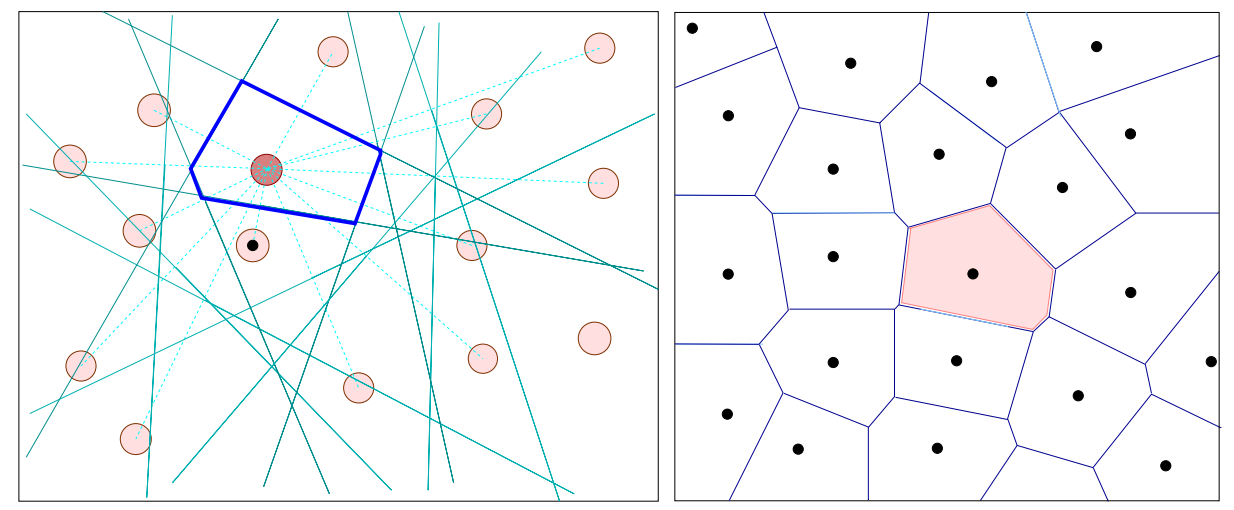

Figure 12: Construction of a Voronoi cells and a Voronoi tiling

It is known that such polyhedra tile the space. Then, since $\mathcal{L}$ has finite type, there is only finitely many Voronoi cells modulo translation. Each such cell will be decorated by the family of its neighbours: a Voronoi cell $V_{y}$ is a neighbour of $V_{x}$ if $\overline{V_{x}} \cap \overline{V_{y}} \neq \emptyset$. A prototile is the equivalence class of a decorated Voronoi cell modulo translations. Then $B_{0}$ is obtained from the disjoint union of the prototiles by gluing two such prototiles $T_{1}$ and $T_{2}$ along one of their face, whenever there is a region in the tiling where two tiles equivalent to $T_{1}$ and $T_{2}$ respectively, are touching along the corresponding face. The decorated Voronoi tiles of $\mathcal{L}$ will be called 0 -tiles.

In general there is no natural inflation rule in a tiling. But it is possible to proceed as follows. Let one of the prototiles $T$ be chosen. And let $\mathcal{L}_{T}$ be the subset of $\mathcal{L}$ made of points with Voronoi cell equivalent to $T$. Thanks to $\mathcal{L}$ being repetitive, $\mathcal{L}_{T}$ is itself a 
Delone set of finite type. It is then possible to build the corresponding Voronoi cells of $\mathcal{L}_{T}$. It will be convenient to substitute to each such cell, the polyhedron $V_{x}^{(1)}$ obtained as the union of the 0-tiles intersecting it, with some convention at the boundary, decorated by the 0 -tiles that touch it from the outside. This will be a 1-tile. Then $B_{1}$ is built in the same way as $B_{0}$. Moreover each 1 -tile is tiled by a family of 0 -tiles, so that the map $f_{0}: B_{1} \mapsto B_{0}$ is well defined as in the octagonal case. The construction of $B_{1}$ from $B_{0}$, can be repeated to get again a projective family of BOF, as in the previous example, and so to the space $\Omega$ together with the $\mathbb{R}^{d}$ action. And this is the interpretation of the Theorem 2.

\section{The Gap Labelling Theorems}

The Shubin formula for electrons or phonons, given by eq. $(22,23)$, shows that the integrated density of electronic states or of phonon modes are given in term of the trace of a spectral projection of a self adjoint operator affiliated to the $C^{*}$-algebra $\mathcal{A}$ of interest. In the electronic case, this operator is the Schrödinger Hamiltonian $H$, which is unbounded, while in the phonon case, it is the mode operator $K$ which belongs to $\mathcal{A}$. There are many situations in which either $H$ or $K$ may have a spectral gap. If this happens, then the spectral projection $\chi(H<E)$ or $\chi\left(K<\tilde{\omega}^{2}\right)$ becomes elements of $\mathcal{A}$ whenever $E$ or $\tilde{\omega}^{2}$ belong to a gap. Since the trace of a projection $P \in \mathcal{A}$ does not change by a unitary transformation, the value of this trace depends only upon the unitary equivalence class of the projection $P$. The notion of unitary equivalence is, however, meaningless in general in a $C^{*}$-algebra since it may not have a unit. This is why it is better to use the von Neumann definition, namely [87]

Definition 12 Two projections $P$ and $Q$ of a $C^{*}$-algebra $\mathcal{A}$ are equivalent, and it is denoted by $P \approx Q$ whenever there is $U \in \mathcal{A}$ such that $U U^{*}=P$ and $U^{*} U=Q$.

As a matter of fact, there are not so many such equivalence classes, more precisely [87]

Theorem 9 In a separable $C^{*}$-algebra, the family of equivalence classes of projections is at most countable.

A consequence is that the trace of a projection belongs to a countable subset of $\mathbb{R}_{+}$. Such numbers will be called gap labels. The question is whether it is possible to compute this subset. The answer is yes and this is the purpose of the various versions of the gap labelling theorem to do so. The main tool is that the set of equivalence classes of projections may be enlarged in a canonical way to become a discrete abelian group, called $K_{0}(\mathcal{A})$ or the $K$-theory group.

\subsection{K-theory}

This section is devoted to a short review of $K$-theoryMorita $[26,114]$. 


\subsubsection{The Group $K_{0}$}

The set of equivalence classes of projections in $\mathcal{A}$ will be denoted by $\mathcal{P}(\mathcal{A})$, and the equivalence class of $P$ by $[P]$. Two projections $P$ and $Q$ are orthogonal whenever $P Q=$ $Q P=0$. Then $P+Q$ is a new projection, called the direct sum of $P$ and $Q$, denoted by $P \oplus Q$.

Proposition 9 Let $\mathcal{A}$ be a separable $C^{*}$-algebra. Let $P$ and $Q$ be two projections in $\mathcal{A}$. Then the equivalence class of their direct sum, if it exists, depends only upon the equivalence classes of $P$ and of $Q$. In particular, a sum is defined on the set $\Theta$ of pairs $([P],[Q])$ in $\mathcal{P}(\mathcal{A})$, such that there are $P^{\prime} \approx P$ and $Q^{\prime} \approx Q$ with $P^{\prime} Q^{\prime}=Q^{\prime} P^{\prime}=0$, by $[P]+[Q]=\left[P^{\prime} \oplus Q^{\prime}\right]$. This composition law is commutative and associative.

The main problem is that the direct sum may not be everywhere defined. To overcome this difficulty, $\mathcal{A}$ is replaced by its stabilization $\mathcal{A} \otimes \mathcal{K}$, where $\mathcal{K}$ is the algebra of compact operators. A $C^{*}$-algebra $\mathcal{A}$ is stable if $\mathcal{A}$ and $\mathcal{A} \otimes \mathcal{K}$ are isomorphic. For any $C^{*}$-algebra $\mathcal{A}, \mathcal{A} \otimes \mathcal{K}$ is always stable, because $\mathcal{K} \otimes \mathcal{K} \cong \mathcal{K}$.

Definition 13 Two $C^{*}$-algebras $\mathcal{A}$ and $\mathcal{B}$ are Morita equivalent whenever $\mathcal{A} \otimes \mathcal{K}$ is isomorphic to $\mathcal{B} \otimes \mathcal{K}$.

Proposition 10 Given any pair $P$ and $Q$ of projections in $\mathcal{A} \otimes \mathcal{K}$, there is always a pair $P^{\prime}, Q^{\prime}$ of mutually orthogonal projections in $\mathcal{A} \otimes \mathcal{K}$ such that $P^{\prime} \approx P$ and $Q^{\prime} \approx Q$. Therefore the sum $[P]+[Q]=\left[P^{\prime} \oplus Q^{\prime}\right]$ is always defined.

In this way, if $\mathcal{A}$ is a stable algebra, the set $\mathcal{P}(\mathcal{A})$ of equivalence classes of projections is an Abelian monoid with neutral element given by the class of the zero projection. If $\mathcal{A}$ is not stable, $\mathcal{P}(\mathcal{A})$ will be replaced by $\mathcal{P}(\mathcal{A} \otimes \mathcal{K})$. The Grothendieck construction gives a canonical way to construct a group from such a monoid. This is a direct generalization of the construction of the group of integers $\mathbb{Z}$ from $\mathbb{N}$. The formal difference $[P]-[Q]$ is defined as the equivalence class of pairs $([P],[Q]) \in \mathcal{P}(\mathcal{A} \otimes \mathcal{K}) \times \mathcal{P}(\mathcal{A} \otimes \mathcal{K})$ under the relation

$$
([P],[Q]) \mathfrak{R}\left(\left[P^{\prime}\right],\left[Q^{\prime}\right]\right) \Leftrightarrow \exists[S] \in \mathcal{P}(\mathcal{A} \otimes \mathcal{K}) ; \quad[P]+\left[Q^{\prime}\right]+[S]=\left[P^{\prime}\right]+[Q]+[S] .
$$

The corresponding quotient is the Abelian group $K_{00}(\mathcal{A})=\mathcal{P}(\mathcal{A} \otimes \mathcal{K}) \times \mathcal{P}(\mathcal{A} \otimes \mathcal{K}) / \mathfrak{R}$. Whenever $\mathcal{A}$ is unital, $K_{0}(\mathcal{A}):=K_{00}(\mathcal{A})$. Otherwise, $\mathcal{A}$ must be enlarged to $\mathcal{A}^{+}$obtained by adjoining a unit, so that $\mathcal{A}$ becomes a two-sided closed ideal of $\mathcal{A}^{+}$. The quotient map $\pi: \mathcal{A}^{+} \rightarrow \mathcal{A}^{+} / \mathcal{A}$ induces a group homomorphism $\pi_{*}: K_{00}\left(\mathcal{A}^{+}\right) \rightarrow K_{00}\left(\mathcal{A}^{+} / \mathcal{A}\right)$, the kernel of which being the group $K_{0}(\mathcal{A})$ (see [26] for details). It leads to:

Proposition 11 Let $\mathcal{A}$ be a separable $C^{*}$-algebra. 
(i) The set $K_{0}(\mathcal{A})$ is countable and has a canonical structure of Abelian group.

(ii) Any *-isomorphism $\varphi: \mathcal{A} \mapsto \mathcal{B}$ between $C^{*}$-algebras induces a group homomorphism $\varphi_{*}: K_{0}(\mathcal{A}) \mapsto K_{0}(\mathcal{B})$, so that $K$ becomes a covariant functor from the category of $C^{*}$-algebras into the category of Abelian discrete groups.

(iii) Any trace $\mathcal{T}$ on $\mathcal{A}$ defines in a unique way a group homomorphism $\mathcal{T}_{*}$ from $K_{0}(\mathcal{A})$ to $\mathbb{R}$ such that if $P$ is a projection on $\mathcal{A}, \mathcal{T}(P)=\mathcal{T}_{*}([P])$ where $[P]$ is the class of $P$ in $K_{0}(\mathcal{A})$.

(iv) If $\mathcal{A}$ and $\mathcal{B}$ are two Morita equivalent $C^{*}$-algebras then $K_{0}(\mathcal{A})$ and $K_{0}(\mathcal{B})$ are isomorphic.

\subsubsection{Higher $K$-groups and exact sequences}

The explicit computation of $K$-groups can be performed using the methods developed in homological algebra. The main tools are exact sequences and spectral sequences. However, these methods require introducing higher order $K$-groups. Let $\mathcal{A}$ be a $C^{*}$-algebra and let $G L_{n}(\mathcal{A})$ be the group of invertible elements of the algebra $M_{n}(\mathcal{A})$. (when $\mathcal{A}$ is non-unital, $\left.G L_{n}(\mathcal{A})=\left\{u \in G L_{n}\left(\mathcal{A}^{+}\right) ; u \equiv \mathbf{1}_{n} \bmod M_{n}(\mathcal{A})\right\}\right) . G L_{n}(\mathcal{A})$ is embedded as a subgroup of $G L_{n+1}(\mathcal{A})$ using

$$
\left(\begin{array}{cc}
G L_{n}(\mathcal{A}) & 0_{n}^{*} \\
0_{n} & 1
\end{array}\right)
$$

(with $0_{n}=(0 \ldots 0)$ ). Let $G L_{\infty}(\mathcal{A})$ be the inductive limit of $G L_{n}(\mathcal{A})$, namely the norm closure of their union, and let $\left[G L_{\infty}(\mathcal{A})\right]_{0}$ be the connected component of the identity in $G L_{\infty}(\mathcal{A}) . K_{1}$ is defined as follows:

$$
K_{1}(\mathcal{A})=G L_{\infty}(\mathcal{A}) / G L_{\infty}(\mathcal{A})_{0}=\lim _{\rightarrow}\left\{G L_{n}(\mathcal{A}) /\left[G L_{n}(\mathcal{A})\right]_{0}\right\}
$$

If $\mathcal{A}$ is separable, then $K_{1}(\mathcal{A})$ is countable, since nearby invertible elements are in the same component. For $u \in G L_{n}(\mathcal{A})$, let $[u]$ be its class in $K_{1}(\mathcal{A})$. The relation $[u][v]=$ $[\operatorname{diag}(u, v)]$ defines a product in $K_{1}(\mathcal{A})$. Then [26]

Proposition $12 K_{1}(\mathcal{A})$ is an Abelian group.

The suspension of $\mathcal{A}$ is the $C^{*}$-algebra $S \mathcal{A}$ of continuous functions $f: \mathbb{R} \rightarrow \mathcal{A}$ vanishing at $\pm \infty$, endowed with point-wise addition, multiplication and adjoint, and the sup-norm. Hence $S \mathcal{A} \cong C_{0}(\mathbb{R}) \otimes \mathcal{A}$. Then $[26]$

Theorem $10 K_{1}(\mathcal{A})$ is canonically isomorphic to $K_{0}(S \mathcal{A})$. 
Therefore we can also define higher $K$-groups by

$$
K_{2}(\mathcal{A})=K_{1}(S \mathcal{A})=K_{0}\left(S^{2} \mathcal{A}\right), \ldots, K_{n}(\mathcal{A})=\cdots=K_{0}\left(S^{n} \mathcal{A}\right) .
$$

Theorem 11 (Bott Periodicity) $K_{0}(\mathcal{A}) \cong K_{2}(\mathcal{A})$. More precisely $K_{0}(\mathcal{A})$ is isomorphic to the group $\pi_{1}\left(G L_{\infty}(\mathcal{A})\right)$ of homotopy classes of closed paths in $G L_{\infty}(\mathcal{A})$. Furthermore, if $\mathcal{T}$ is a trace on $\mathcal{A}$ and if $t \in[0,1] \rightarrow U(t)$ is a closed path in $G L_{\infty}(\mathcal{A})$ [37]:

$$
\mathcal{T}_{*}([U])=\frac{1}{2 \pi i} \int_{[0,1]} d t \mathcal{T}\left(U(t)^{-1} U^{\prime}(t)\right),
$$

where $\mathcal{T}_{*}$ is the map induced by $\mathcal{T}$ on $K_{0}(\mathcal{A})$.

$K_{i}$ is a covariant functor with the following properties:

Theorem 12 Let $\mathcal{J}, \mathcal{A}, \mathcal{A}_{n}, \mathcal{B}$ be $C^{*}$-algebras and $n, i$ non-negative integers:

(i) If $f: \mathcal{A} \rightarrow \mathcal{B}$ is a *-homomorphism, then $f$ induces a group homomorphism $f_{*}$ : $K_{i}(\mathcal{A}) \rightarrow K_{i}(\mathcal{B})$. Then $i d_{*}=i d$, and $(f \circ g)_{*}=f_{*} \circ g_{*}$.

(ii) $K_{i}\left(\bigoplus_{n} \mathcal{A}_{n}\right) \cong \bigoplus_{n} K_{i}(\mathcal{A})$

(iii) If $\mathcal{A}$ is the inductive limit of the sequence $\left(\mathcal{A}_{n}\right)_{n>0}$ of $C^{*}$-algebras then $K_{i}(\mathcal{A})$ is the inductive limit of the groups $K_{i}\left(\mathcal{A}_{n}\right)$.

(iv) If $\phi: \mathcal{J} \rightarrow \mathcal{A}$, and $\psi: \mathcal{A} \rightarrow \mathcal{B}$ are $*$-homomorphisms such that the sequence

$$
0 \rightarrow \mathcal{J} \rightarrow \mathcal{A} \rightarrow \mathcal{B} \rightarrow 0
$$

be exact, there is a six-term exact sequence of the form:

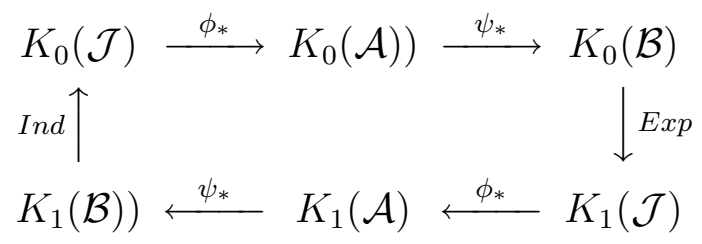

In the previous theorem, Ind et Exp are the connection automorphisms defined as follows (whenever $\mathcal{A}$ is unital): let $P$ be a projection in $\mathcal{B} \otimes \mathcal{K}$, and let $A$ be a self-adjoint element of $\mathcal{A} \otimes \mathcal{K}$ such that $\psi \otimes i d(A)=P$. Then $\psi \otimes i d\left(e^{2 \imath \pi A}\right)=e^{2 \imath \pi P}=\mathbf{1}$, so that $B=e^{2 \imath \pi A} \in(\mathcal{J} \otimes \mathcal{K})^{+}$and is unitary in $(\mathcal{J} \otimes \mathcal{K})^{+}$. The class of $B$ gives an element of $K_{1}(\mathcal{J})$ which is, by definition, $\operatorname{Exp}([P])$. In much the same way, let now $U$ be an unitary element of $\mathbf{1}+(\mathcal{B} \otimes \mathcal{K})$. Without loss of generality it is the image under $\psi \otimes i d$ of a partial isometry $W$ in $(\mathcal{A} \otimes \mathcal{K})$. Then $\operatorname{Ind}([U])$ is the class of $\left[W W^{*}\right]-\left[W^{*} W\right]$ in $K_{0}(\mathcal{J})$. These definitions actually make sense. 


\subsubsection{The Connes-Thom isomorphism}

The $C^{*}$-algebra of a dynamical system introduced in Section 2.6 is a special case of the $C^{*}$-crossed product construction. Let $\mathcal{A}$ be a $C^{*}$-algebra, $\mathbb{G}$ be a locally compact group, and $\alpha$ be a continuous homomorphism from $\mathbb{G}$ into $\operatorname{Aut}(\mathcal{A})$ (namely the group of *-automorphisms of $\mathcal{A}$ endowed with the topology of point-wise norm-convergence). A covariant representation of the triple $(\mathcal{A}, \mathbb{G}, \alpha)$ is a pair of representations $(\Pi, \rho)$ of $\mathcal{A}$ and $\mathbb{G}$ on the same Hilbert space such that $\rho(g) \Pi(a) \rho(g)^{*}=\Pi\left(\alpha_{g}(a)\right)$ for all $a \in \mathcal{A}$ and $g \in \mathbb{G}$. Each covariant representation of $(\mathcal{A}, \mathbb{G}, \alpha)$ gives a representation of the twisted convolution algebra $C_{c}(\mathbb{G}, A)$ by integration (compare with Section 2.6), and hence a pre- $C^{*}$-norm on this $*$-algebra. The supremum of all these norms is a $C^{*}$-norm, and the completion of $\mathcal{C}_{c}(\mathbb{G}, A)$ with respect to this norm is called the crossed product of $\mathcal{A}$ by $\mathbb{G}$ under the action $\alpha$, denoted by $A \rtimes_{\alpha} \mathbb{G}$. The ${ }^{*}$-representations of $A \rtimes_{\alpha} \mathbb{G}$ are in natural one-to-one correspondence with the covariant representations of the dynamical system $(\mathcal{A}, \mathbb{G}, \alpha)$.

Theorem $13[37] K_{i}\left(A \rtimes_{\alpha} \mathbb{R}\right) \cong K_{1-i}(A), \quad$ for $i=0,1$.

\subsubsection{The Pimsner \& Voiculescu exact sequence}

The following result can be found in [91]

Theorem 14 Let $\mathcal{A}$ be a separable $C^{*}$-algebra, and let $\alpha$ be a ${ }^{*}$-automorphism of $\mathcal{A}$. There exists a six-term exact sequence:

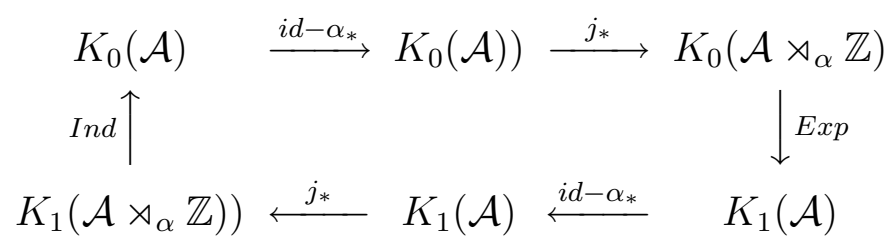

where $j$ is the canonical injection of $\mathcal{A}$ into the crossed product.

\subsubsection{Morita equivalence}

In Section 4.1.1 the notion of Morita equivalence was defined. Namely, $C^{*}$-algebras $\mathcal{A}, \mathcal{B}$ are called Morita equivalent whenever $\mathcal{A} \otimes \mathcal{K} \simeq \mathcal{B} \otimes \mathcal{K}$, if $\mathcal{K}$ is the algebra of compact operators. In Prop. 11.iv, it was shown that if $\mathcal{A}$ and $\mathcal{B}$ are Morita equivalent, then they have same $K$-groups. The following theorem will be used here (see for instance[41])

Theorem 15 Let $\Omega$ be a compact metrizable space endowed with an action of $\mathbb{R}^{d}$ by homeomorphisms. Let $X \subset \Omega$ be a transversal. Let $\Gamma(X)$ be the groupoid of the transversal. Then the $C^{*}$-algebras $\mathcal{A}=\mathcal{C}(\Omega) \rtimes \mathbb{R}^{d}$ and $\mathcal{B}=C^{*}(\Gamma(X))$ are Morita equivalent. 


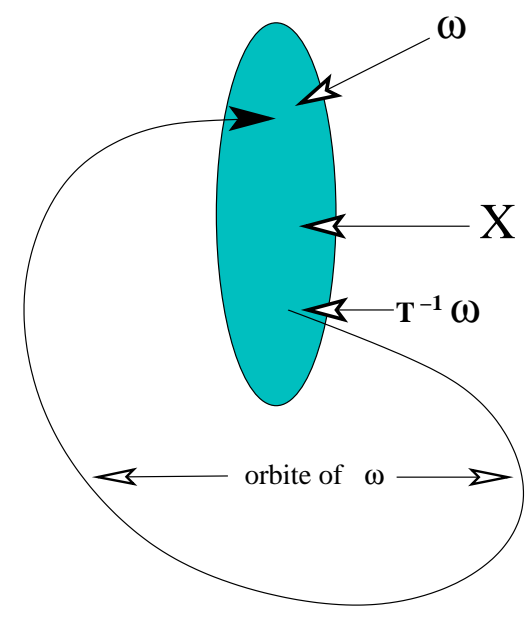

Figure 13: The Poincaré first return map

In particular, whenever $d=1$, a transversal is called a Poincaré section. The construction of the first return map $\mathrm{T}$, also called Poincaré map, shows that the groupoid $\Gamma(X)$ is obtained as the action of $\mathbb{Z}$ on $X$ defined by $\mathrm{T}$ (see Fig. 13).

Hence, the $C^{*}$-algebra of the transversal is nothing but $\mathcal{B}=\mathcal{C}(X) \rtimes \mathbb{Z}$.

\subsection{Gap Labels}

Let be a self-adjoint operator affiliated to $\mathcal{A}$ and satisfying Shubin's formula (22). Let $\mathfrak{g}$ be a gap in the spectrum $\mathrm{Sp} H$ of $H$. The integrated density of states (IDOS) is constant on this gap. Let $\mathcal{N}(\mathfrak{g})$ be its value there. Moreover, if $E \in \mathfrak{g}$, the characteristic function $x \in \mathrm{Sp} H \mapsto \chi(x \leq E) \in \mathbb{R}$ is continuous on $\mathrm{Sp} H$ and does not depend upon the choice of $E \in \mathfrak{g}$. Therefore the spectral projection $\chi(H \leq E)$ is an element $P(\mathfrak{g}) \in \mathcal{A}$ which depends only upon the gap. Hence, it defines an element $n(\mathfrak{g})=[P(\mathfrak{g})] \in K_{0}(\mathcal{A})$. The Shubin formula implies:

$$
\mathcal{N}(\mathfrak{g})=\mathcal{T}_{\mathbb{P}}(P(\mathfrak{g}))=\mathcal{T}_{\mathbb{P} *}(n(\mathfrak{g})),
$$

namely, the IDOS on gaps is a number that belongs to the image of the $K_{0}$-group by the trace. Since this group is countable such numbers belong to a countable subgroup of $\mathbb{R}$. At last since a projection satisfies $0 \leq P \leq \mathbf{1}$, the trace of a projection satisfies $o \leq \mathcal{T}_{\mathbb{P}}(P) \leq \mathcal{T}_{\mathbb{P}}(\mathbf{1})$. Hence:

Theorem 16 Abstract Gap Labelling Theorem[12, 13]

Let $H$ be a self-adjoint operator affiliated to $\mathcal{A}$ and satisfying Shubin's formula. Then:

(i) For any gap $\mathfrak{g}$ in the spectrum of $H$, the value of the IDOS of $H$ on $\mathfrak{g}$ belongs to the countable set of real numbers $\mathcal{T}_{*}\left(K_{0}(\mathcal{A})\right) \cap[0, \mathcal{T}(\mathbf{1})]$. 


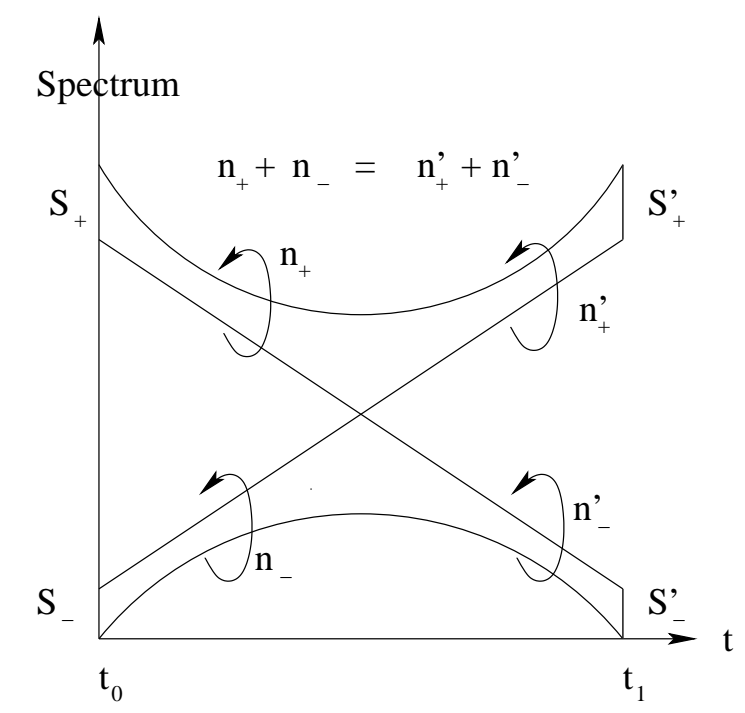

Figure 14: Conservation rules for gap labels (Theorem 16 (v))

(ii) The equivalence class $n(\mathfrak{g})=[P(\mathfrak{g})] \in K_{0}(\mathcal{A})$, gives a labelling which is invariant under norm perturbations of the Hamiltonian $H$ within $\mathcal{A}$.

(iii) If $S \subset \mathbb{R}$ is a closed and open subset in $\operatorname{Sp} H$, then $n_{S}=\left[P_{S}\right] \in K_{0}(\mathcal{A})$, where $P_{S}$ is the eigenprojection of $H$ corresponding to $S$, is a labelling for each such part of the spectrum.

Let $t \in \mathbb{R} \rightarrow H(t)$ be a continuous family of self-adjoint operators (in the norm-resolvent topology) with resolvent in $\mathcal{A}$.

(iv) (homotopy invariance) The gap edges of $H(t)$ are continuous and the labelling of a gap $\{\mathfrak{g}(t)\}$, is independent of $t$ as long as the gap does not close.

(v) (additivity) (see Fig. 14) If for $t \in\left[t_{0}, t_{1}\right]$, the spectrum of $H$ contains a clopen subset $S(t)$ such that $S(0)=S_{+} \cup S_{-}$and $S(1)=S_{+}^{\prime} \cup S_{-}^{\prime}$ where $S_{ \pm}$and $S_{ \pm}^{\prime}$ are clopen sets in $\operatorname{spec}\left(H\left(t_{0}\right)\right)$ and $\operatorname{spec}\left(H\left(t_{1}\right)\right)$ respectively, then $n_{S_{+}}+n_{S_{-}}=n_{S_{+}^{\prime}}+n_{S_{-}^{\prime}}$.

Example 6 The first example of such a gap labelling, using $K$-theory, was the case of the Harper model [12] using the results of Rieffel and Pimsner \& Voiculescu [92, 97] on the irrational rotation algebra. The Harper model is the Hamiltonian describing the motion of a $2 D$-electron on the square lattice and submitted to a uniform magnetic field perpendicular to the lattice like in Fig. 15. The Hilbert space of electronic states is $\ell^{2}\left(\mathbb{Z}^{2}\right)$. The energy operator is reduced to its discrete kinetic term:

$$
H=U_{1}+U_{1}^{-1}+U_{2}+U_{2}^{-1}
$$




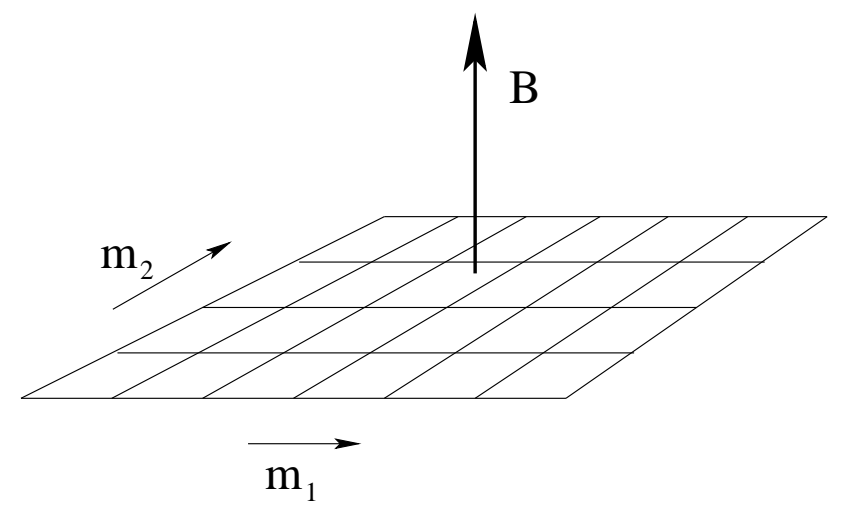

Figure 15: A square lattice in a uniform magnetic field

where $U_{1}, U_{2}$ are the magnetic translations in the directions of the two axis [119] defined by

$$
U_{i} \psi(\vec{m})=e^{\imath e / \hbar \oint_{\vec{m}-\vec{e}_{i}}^{\vec{m}} \vec{A} \cdot d \vec{\ell}} \psi\left(\vec{m}-\vec{e}_{i}\right), \quad \vec{m}=\left(m_{1}, m_{2}\right) \in \mathbb{Z}^{2} \quad \vec{e}_{1}=(1,0) \quad \vec{e}_{2}=(0,1)
$$

It is elementary to check that

$$
U_{i}^{-1}=U_{i}^{*}(i=1,2) \quad U_{1} U_{2}=e^{2 \imath \pi \alpha} U_{2} U_{1}, \quad \alpha=\frac{\phi}{\phi_{0}},
$$

where $\phi$ is the magnetic flux through the unit cell, whereas $\phi_{0}=h / e^{2}$ is the flux quantum (here $h=2 \pi \hbar$ ). The $C^{*}$-algebra generated by two unitaries satisfying (36) is denoted by $\mathcal{A}_{\alpha}$ and is called the rotation algebra [97]. It is easy to see that the $C^{*}$-algebra generated by one unitary, say $U_{2}$, is isomorphic to $\mathcal{C}(\mathbb{T})$ if $U_{2}$ is identified with the trigonometric monomial $x \in \mathbb{T} \mapsto e^{\imath x} \in \mathbb{C}$. The commutaion rule given in (36) implies that $U_{1}$ acts on $\mathcal{C}(\mathbb{T})$ through this isomorphism as

$$
U_{1} f U_{1}^{-1}(x)=f(x+2 \pi \alpha) .
$$

Hence, $U_{1}$ is the generator of the $\mathbb{Z}$-action $\alpha: x \in \mathbb{T} \mapsto x+2 \pi \alpha(\bmod 2 \pi)$, so that $\mathcal{A}_{\alpha}$ is isomorphic to the crossed product $\mathcal{C}(\mathbb{T}) \rtimes_{\alpha} \mathbb{Z}$. It is clear that within the algebra $\mathcal{C}(\mathbb{T}) \otimes M_{n}(\mathbb{C})$ the automorphism $\alpha(F)(x)=F(x+2 \pi \alpha)$ is homotopic to the identity, so that the action of $\alpha$ on the $K$-group is trivial so that $\alpha_{*}-i d=0$. Moreover, the $K$-groups of the torus are $K_{0} \simeq \mathbb{Z} \simeq K_{1}$. The generator of $K_{0}$ is the function $f(x)=1$, whereas $U_{2}$ generates $K_{1}$. The Pimsner-Voiculescu exact sequence (Theorem 14), splits into two independent short exact sequences

$$
0 \mapsto \mathbb{Z} \stackrel{i_{*}}{\longmapsto} K_{i}(\mathcal{A}) \stackrel{\partial}{\longmapsto} \mathbb{Z} \mapsto 0, \quad i=0,1
$$


where $i_{*}$ is the canonical injection of $\mathcal{C}(\mathbb{T})$ into $\mathcal{A}_{\alpha}=\mathcal{C}(\mathbb{T}) \rtimes_{\alpha} \mathbb{Z}$ whereas $\partial$ is the connecting homomorphism. Since $\mathbb{Z}$ is a free group, the only solution is $K_{i}(\mathcal{A}) \simeq \mathbb{Z}^{2}$ for $i=0,1$.

In addition, the trace per unit area satisfies

$$
\mathcal{T}\left(U_{1}^{n_{1}} U_{2}^{n_{2}}\right)=0 \quad \text { if } \quad n_{1}=n_{2}=0, \quad \mathcal{T}(\mathbf{1})=1
$$

Since 1 is one generator of $K_{0}(\mathcal{A})$, it is sufficient to built a projection non equivalent to 1 to get another generator. The following result was proved by [92] and can be proved nowadays in several other ways

Theorem 17 If $\alpha$ is an irrational number, and if $P \in \mathcal{A}_{\alpha}$ is a projection, there is a unique integer $n$ such that

$$
\mathcal{T}(P)=n \alpha-[n \alpha]
$$

where $[x]$ denote the integer part of $x$.

These integers was recognized by Claro and Wannier [35] in 1978 (see Fig. 16: the horizontal axis corresponds to the spectrum of $H$, the vertical axis corresponds to the value of $\alpha$ ), on the basis of the numerical calculation by Hofstadter [63] (see Fig. 1). Eventually these integers are the one occuring in the Quantum Hall effect (see Section 5).

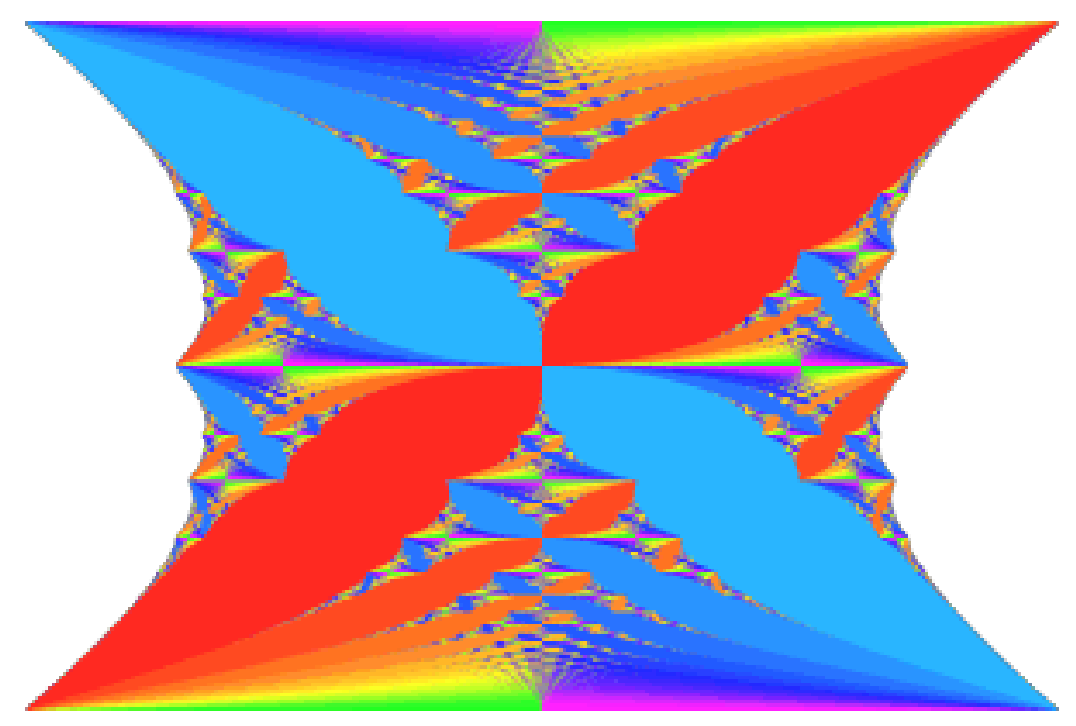

Figure 16: Gap labels for the Harper model: each color corresponds to an integer [84] 


\subsection{Computing Gap Labels}

The first systematic computation of gap labels was performed for $1 D$ systems in $[16,21]$. Let $\Omega$ be the Hull of the system and $X$ its transversal. In Section 4.1.5 it has been shown that the $K$-group of $\mathcal{A}=\mathcal{C}(\Omega) \rtimes \mathbb{R}$ is the same a the one of the groupoid of the transversal $\mathcal{C}(X) \rtimes \mathbb{Z}$ where $\mathbb{Z}$ acts through the Poincaré map T. The Pimsner-Voiculescu exact sequence, defined in Section 4.1 .4 allows to compute the $K$-group from the topology of $X$. The simplest case occurs whenever $X$ is a Cantor set.

Definition 14 Let $G$ be an Abelian group and let $T: G \mapsto G$ be a group isomorphism. The set $\mathcal{E}=\{g \in G ; \exists h \in G, g=h-T(h)\}$ is a subgroup. The set $G^{T}=\{g \in G ; T(g)=$ $g\}$ is called the group of invariants whereas $G_{T}=G / \mathcal{E}$ is called the groups of co-invariants.

Theorem 18 [16, 21] Let $X$ be a totally disconnected compact metrizable space, endowed with a $\mathbb{Z}$-action $\mathrm{T}$ and let $\mathcal{B}=\mathcal{C}(X) \rtimes \mathbb{Z}$ be the corresponding $C^{*}$-algebra. Let $\mathrm{T}$ also denotes the induced action on the abelian group $\mathcal{C}(X, \mathbb{Z})$ defined by $\mathrm{T} f(x)=f\left(\mathrm{~T}^{-1} x\right)$. Then

(i) $K_{1}(\mathcal{B})$ is isomorphic to the group of invariant $\mathcal{C}(X, \mathbb{Z})^{\mathrm{T}}$. In particular, if $\mathrm{T}$ is topologically transitive, namely if there is one dense orbit, $K_{1}(\mathcal{B}) \simeq \mathbb{Z}$.

(ii) $K_{0}(\mathcal{B})$ is isomorphic to the group of co-invariants $\mathcal{C}(X, \mathbb{Z})_{\mathrm{T}}$.

(iii) Let $\mathbb{P}$ be a T-invariant ergodic probability measure on $X$ and let $\mathcal{T}_{\mathbb{P}}$ be the corresponding trace on $\mathcal{B}$. Then, the set of gap labels of $\mathcal{B}$, namely the image of $K_{0}(\mathcal{B})$ by $\mathcal{T}_{\mathbb{P}}$ is the countable subgroup $\mathbb{P}(\mathcal{C}(X, \mathbb{Z}))$ of $\mathbb{R}$.

Example 7 The main application is the following. Let $\mathfrak{A}$ be a finite subset of $\mathbb{R}$ that will be called an alphabet. The elements of $\mathfrak{A}$ will be called letters. A word is a finite sequence of letters. The set of words is denoted by $\mathfrak{W}$. The length of a word $w$ is the number, denoted by $|w|$, of its letters. If $v$ and $w$ are two words, $v w$ denotes the word obtained by concatenation, namely by associating the list of letters appearing in $v$ followed by the one in $w$. Then $|v w|=|v|+|w|$. Let now $\mathfrak{A}^{\mathbb{Z}}$ be the set of doubly infinite sequence of letters $\underline{u}=\left(u_{i}\right)_{i \in \mathbb{Z}}$ with $u_{i} \in \mathfrak{A}$ for every $i \in \mathbb{Z}$. $\mathbb{Z}$ acts on $\mathfrak{A}^{\mathbb{Z}}$ through the bilateral shift $(\mathrm{T} \underline{u})_{i}=u_{i-1}$. Given $\underline{u}^{(0)} \in \mathfrak{A}$ let $X$ be the closure in $\mathfrak{A}^{\mathbb{Z}}$ of the set $\left\{\mathrm{T}^{n} \underline{u}^{(0)} ; n \in \mathbb{Z}\right\}$ of the shifted of $\underline{u}^{(0)}$. X is called the Hull of $\underline{u}^{(0)}$. A cylinder set is a clopen set of the form $\mathcal{U}_{w, n}=\left\{\underline{u} \in X ; u_{n+i}=w_{i}, 1 \leq i \leq|\underline{w}|\right\}$, for some $n \in \mathbb{Z}$ and some word $w$. Cylinder sets form the basis of the topology of $X$. A cylindrical function $f: X \mapsto \mathbb{C}$ on $X$ is a function for which there is $N \in \mathbb{N}$ such that, for $\underline{u} \in X, f(u)$ depends only on $\left(u_{-N}, u_{N-1}, \cdots, u_{N-1}, u_{N}\right)$. By definition of the product topology, any continuous function $f \in \mathcal{C}(X)$ can be uniformly approximated by a sequence of cylindrical function. As a consequence, it is possible to show that any integer valued continuous function $g$ is a finite sum of characterisic function of cylinder sets. Given any T-invariant ergodic 
probability measure $\mathbb{P}$ on $X$, the probability $\mathbb{P}\left(\mathcal{U}_{w, n}\right)$ of the cylinder set $\mathcal{U}_{w, n}$ does not depend on $n$ and is nothing but the occurence probability of the word $w$ in the sequence $\underline{u}^{(0)}$. Hence, Theorem 18 implies

Proposition 13 Let $\mathfrak{A}$ be an alphabet and let $\underline{u}^{(0)}$ be a doubly infinite sequence of letters in $\mathfrak{A}$, and let $X$ be its Hull. Let $\mathbb{P}$ be a $\mathrm{T}$ invariant ergodic probability on $X$. Then the set of gap labels of the algebra $\mathcal{B}=\mathcal{C}(X) \rtimes \mathbb{Z}$ is nothing but the $\mathbb{Z}$-module $Z_{\underline{u}^{(0)}}$ generated by the occurence probabilities of finite words contained in the sequence $\underline{u}^{(0)}$.

Let now $H$ be a selfadjoint operator on $\ell^{2}(\mathbb{Z})$ defined by

$$
H \psi(n)=\sum_{m \in \mathbb{Z}} h\left(\mathrm{~T}^{-m} \underline{u}, n-m\right) \psi(m),
$$

where, for each $n \in \mathbb{Z}$, the map $h_{n}: \underline{u} \mapsto h(\underline{u}, n) \in \mathbb{C}$ is continuous and satisfies: (i) $\overline{h(\underline{u}, n)}=h\left(\mathrm{~T}^{-n} \underline{u},-n\right)$ and (ii) $\sup _{\underline{u} \in X} \sum_{n \in \mathbb{Z}}|h(\underline{u}, n)|<\infty$. Then $H \in \mathcal{B}$ and it IDOS on gaps takes on values in $Z_{\underline{u}}(0)$.

A substitution $\sigma$ is a map $\sigma: \mathfrak{A} \mapsto \mathfrak{W}$. It extends to $\mathfrak{W}$ by concatenation, namely if $w=a_{1} a_{2} \cdots a_{n}$ then $\sigma(w)=\sigma\left(a_{1}\right) \sigma\left(a_{2}\right) \cdots \sigma\left(a_{n}\right)$. It will be assumed that

(i) $\exists a \in \mathfrak{A}$ such that $\sigma(a)=v a w$, for some non empty words $v, w$;

(ii) $\sigma$ is primitive namely, given any pairs of letters $b, c$ there is an $n \in \mathbb{N}$ such that $\sigma^{n}(b)$ contains the letter $c$;

(iii) $\sigma$ is generating, namely, for any letter $b$, the length of $\sigma^{n}(b)$ diverges as $n \rightarrow \infty$. Then, the sequence of words $\sigma^{n}(a)$ converges in $\mathfrak{A}^{\mathbb{Z}}$ to an infinite sequence $\underline{u}^{(0)}$. Such a sequence will be called a substitution sequence. The following is a classical result [94]

Theorem 19 Let $\underline{u}^{(0)}$ be a substitution sequence. Then there is a unique T-invariant ergodic probability measure on its Hull.

Let $M(\sigma)_{b, a}$ be the number of occurences of the letter $b$ in the word $\sigma(a)$. Then $M(\sigma)$ is the matrix of the substitution $\sigma$. Because it is a matrix with nonnegative entries, the Perron-Frobenius Theorem implies that it has a simple eigenvalue $\theta$ equal to its spectral radius, called the Perron-Frobenius eigenvalue, with eigenvector $V=\left(V_{b}\right)_{b \in \mathfrak{A}}$ with positive entries and normalized to $\sum_{b} V_{b}=1$, called the Perron-Frobenius vector. It follows from the definition of this matrix that $v_{b}$ is the occurence probability of the letter $b$ in the substitution sequence.

Let now $\mathfrak{A}_{N}$ be the set of words of length $N$. Then, considering $\mathfrak{A}_{N}$ as a new alphabet, $\sigma$ induces on $\mathfrak{A}_{N}$ a substitution $\sigma_{N}$ defined as follows. If $w$ is a word with $N$ letters and first letter $b$, let $m$ be the length of $\sigma(b)$ and let $\sigma(w)=a_{1} \cdots a_{n}$. Then $\sigma_{N}(w)=\left(a_{1} \cdots a_{N}\right)\left(a_{2} \cdots a_{N+1}\right) \cdots\left(a_{m} \cdots a_{N+m}\right)$. Let then $M_{N}(\sigma)$ be the matrix of this new substitution. Then it follows that $\theta$ is also the Perron-Frobenius eigenvalue of $M_{N}(\sigma)$ [94], for any $N$. Moreover [16, 21] 
Theorem 20 Let $\sigma$ be a substitution satisfying the condition (i),(ii),(iii) above. Let $\underline{u}^{(0)}$ be the corresponding substitution sequence and $X$ be its hull. Then the set of gap labels is given by the $\mathbb{Z}\left[\theta^{-1}\right]$-module generated by the components of the Perron-Frobenius vectors of $M(\sigma)$ and $M_{2}(\sigma)$, where $\theta$ is the Perron-Frobenius eigenvalue.

Example 8 The generalization of the previous example can be described as follows. Let $X$ be a Cantor set endowed with an action of $\mathbb{Z}^{d}$ by homeomorphisms. Let $\mathcal{B}=\mathcal{C}(X) \rtimes \mathbb{Z}^{d}$ be the corresponding $C^{*}$-algebra . Then Forrest and Hunton [53], using the AtiyahHirzebruch spectral sequences [6] and a result by Adams [2], proved the following theorem

Theorem 21 If $X$ is a Cantor set endowed with a minimal action of $\mathbb{Z}^{d}$ by homeomorphisms. Then the group of $K$-theory of the corresponding $C^{*}$-algebra $\mathcal{B}=\mathcal{C}(X) \rtimes \mathbb{Z}^{d}$ is given by

$$
K_{i}(\mathcal{B}) \simeq \bigoplus_{m=0}^{d / 2} H^{2 m+i}\left(\mathbb{Z}^{d}, \mathcal{C}(X, \mathbb{Z})\right),
$$

where $H^{n}\left(\mathbb{Z}^{d}, G\right)$ is the group cohomology of $\mathbb{Z}^{d}$ with coefficients in the abelian group $G$ and $\mathcal{C}(X, \mathbb{Z})$ denotes the abelian group of integer valued continuous functions on $X$.

Let now $\mathbb{P}$ be a $\mathbb{Z}^{d}$-invariant ergodic probability measure on $X$ and let $\mathcal{T}_{\mathbb{P}}$ be the corresponding normalized trace on $\mathcal{B}$. Then it has been proved recently by $[25,68]$ that

Theorem 22 If $X$ is a Cantor set endowed with an action of $\mathbb{Z}^{d}$ by homeomorphisms and if $\mathbb{P}$ is a $\mathbb{Z}^{d}$-invariant ergodic probability measure on $X$, then the set of gap labels, namely the image by the trace $\mathcal{T}_{\mathbb{P}}$ induced by $\mathbb{P}$ on $\mathcal{B}=\mathcal{C}(X) \rtimes \mathbb{Z}^{d}$, is given by $\mathbb{P}(\mathcal{C}(X, \mathbb{Z})$ ).

Example 9 The definition of a finite type repetitive Delone set $\mathcal{L}$ given in Section 2.3, is actually the $d$-dimensional generalization of a bi-infinite sequence of letters. For indeed, thanks to the Voronoi construction (see Section 3.4) the points of $\mathcal{L}$ can be seen as tiles instead and the finite type property means that the number of such tiles is finite modulo translations. A prototile is an equivalence class of tiles modulo translation. Let then $\mathfrak{T}$ be the set of prototiles. This is the $d$-dimensional analog of the alphabet. However, prototiles lead to patches instead of words. A patch is a finite union of tiles of $\mathcal{L}$, but such a notion is too loose. Given $r \geq 0$ an $r$-patch of $\mathcal{L}$ is the finite union $p$ of closed tiles of $\mathcal{L}$ centered at points of a subset of the form $\mathcal{L}(p)=B(0 ; r] \cap(\mathcal{L}-x)$ for some $x \in \mathcal{L}$. Here $B(y ; r]$ denotes the closed ball of radius $r$ centered at $y$. Geometrically an $r$-patch $p$ is a polyhedron. In the following, $\Omega$ will denote the Hull of $\mathcal{L}$ and $X$ its transversal. According to the construction given in Section 2.2, any $\omega \in \Omega$ is a Radon measure on 
$\mathbb{R}^{d}$ supported by a Delone set $\mathcal{L}_{\omega}$, and giving mass one to each point of $\mathcal{L}_{\omega}$. Then $\mathcal{L}_{\omega}$ is itself repetitive and has finite type. Since $\mathcal{L}$ has finite type, it is easy to check that the prototiles and the patches of $\mathcal{L}_{\omega}$ are the same as for $\mathcal{L}$. It is useful to recall that $\omega \in X$ if and only if $\mathcal{L}_{\omega}$ contains the origin.

Let then $\mathfrak{P}(r)$ be the set of $r$-patches of $\mathcal{L}$. Since $\mathcal{L}$ has finite type, $\mathfrak{P}(r)$ is finite so that the map $r \in[0, \infty) \mapsto \mathfrak{P}(r)$ is locally constant and upper semicontinuous. Hence there is an infinite increasing sequence $0=r_{0}<r_{1}<\cdots<r_{n}<r_{n+1}<\cdots$ such that $r_{n} \leq r<r_{n+1} \Rightarrow \mathfrak{P}(r)=\mathfrak{P}\left(r_{n}\right)$. Let $\mathfrak{P}_{n}$ denote $\mathfrak{P}\left(r_{n}\right)$ and let $\mathfrak{P}$ be the union of the $\mathfrak{P}_{n}$ 's. In particular, $\mathfrak{P}_{0}=\mathfrak{T}$. The analogy with the $1 D$ case is now simple. The alphabet $\mathfrak{A}$ is replaced by the set of prototiles $\mathfrak{T}$, the set of words $\mathfrak{W}$ by the set of patches $\mathfrak{P}$ and the bi-infinite sequence $\underline{u}^{(0)}$ of letters by $\mathcal{L}$. Then the Hull of $\underline{u}^{(0)}$ is replaced by the transversal $X$ of $\mathcal{L}$. Cylinder sets are now given by clopen subsets $\mathcal{U}(p, n) \subset X$, with $p \in \mathfrak{P}_{n}$ for some $n \in \mathbb{N}$, where $\mathcal{U}(p, n)$ denotes the set of $\omega \in X$ for which the union of the closed tiles centered on points of $B\left(0 ; r_{n}\right] \cap \mathcal{L}_{\omega}$ is precisely $p$.

In Section 3.4, eq. (31) gives the construction of the Hull from a projective sequence $\ldots \stackrel{f_{n+1}}{\longrightarrow} B_{n+1} \stackrel{f_{n}}{\longrightarrow} B_{n} \stackrel{f_{n-1}}{\longrightarrow} \cdots$ of BOF manifolds from the notion of decorated patches. The following result has been proved in [24] which is a an extenxion of the Forrest-Hunton theorem 21 [53]

Theorem 23 Let $\mathcal{L}$ be a repetitive Delone set of finite type in $\mathbb{R}^{d}$. Let $\Omega$ its Hull and let $\mathcal{A}=\mathcal{C}(\Omega) \rtimes \mathbb{R}^{d}$ be the corresponding Noncommutative Brillouin zone. Then

(i) the group of $K$-theory of $\mathcal{A}$ is given by

$$
K_{i}(\mathcal{A}) \simeq \lim _{n \rightarrow \bigoplus_{m=0}} \bigoplus^{2 m+i}\left(B_{n}, \mathbb{Z}\right)
$$

(ii) The longitudinal homology group group $H_{d}(\Omega)=\lim _{\leftarrow} H_{d}\left(b_{n}, \mathbb{R}\right)$ is canonically ordered with positive cone $H_{d}(\Omega)_{+}$. There is a canonical bijection between $H_{d}(\Omega)_{+}$and the set of $\mathbb{R}^{d}$-invariant positive measure on $\Omega$.

Let now $\mathbb{P}$ be an $\mathbb{R}^{d}$-invariant measure on $\Omega$. It then defines a canonical probability on $X$, called the transverse measure induced by $\mathbb{P}[36,41]$. To build this transverse measure, let a box be defined as a subset of $\Omega$ of the form

$$
\widehat{B}(p, n ; r)=\left\{\omega^{\prime} \in \Omega ; \exists a \in \mathbb{R}^{d},|a|<r, \mathrm{~T}^{-a} \omega^{\prime} \in \mathcal{U}(p, n)\right\},
$$

where $n \in \mathbb{N}, p$ is a patch in $\mathfrak{P}_{n}$ and $r>0$. Then, if $\mathcal{L}$ is $r_{0}$-discrete, the transverse measure is the probability $\mathbb{P}_{X}$ on $X$ uniquely defined by

$$
\mathbb{P}_{X}(\mathcal{U}(p, n))=\frac{\mathbb{P}(\widehat{B}(p, n ; r))}{|B(0 ; r]|}, \quad \forall 0<r<r_{0}
$$


where $|A|$ denotes the Lebesgue measure of $A \subset \mathbb{R}^{d}$. If, in addition, $\mathbb{P}$ is ergodic, then $\mathbb{P}_{X}(\mathcal{U}(p, n))$ is nothing but the occurence probability of the patch $p$ in the tiling associated with $\mathcal{L}$. The following result was proved in [24] and is complementary to $[25,68]$

Theorem 24 Let $\mathcal{L}$ be a repetitive Delone set of finite type in $\mathbb{R}^{d}$. Let $\Omega$ its Hull and let $\mathbb{P}$ be an $\mathbb{R}^{d}$-invariant ergodic measure on $\Omega$. Let $\mathcal{A}=\mathcal{C}(\Omega) \rtimes \mathbb{R}^{d}$ be the corresponding noncommutative Brillouin zone and $\mathcal{T}_{\mathbb{P}}$ the trace induced by $\mathbb{P}$. Then the set of gap labels, namely the image by $\mathcal{T}_{\mathbb{P}}$ of the $K_{0}$-group of $\mathcal{A}$, is the $\mathbb{Z}$-module generated by the occurence probabilities of patches.

\section{The Quantum Hall Effect}

In 1880, E.H. Hall [58] undertook the classical experiment which led to the so-called Hall effect. A century later, von Klitzing and his co-workers [73] showed that the Hall conductivity was quantized at very low temperatures as an integer multiple of the universal constant $e^{2} / h$. Here $e$ is the electron charge whereas $h$ is Planck's constant. This is the Integer Quantum Hall Effect (IQHE). This discovery led to a new accurate measurement of the fine structure constant and a new definition of the standard of resistance [106].

After the works by Laughlin [78] and especially by Kohmoto, den Nijs, Nightingale and Thouless [110] (called $T K N_{2}$ below), it became clear that the quantization of the Hall conductance at low temperature had a geometric origin. The universality of this effect had then an explanation. Moreover, as proposed by Prange [93, 66], Thouless [109] and Halperin [59], the Hall conductance plateaus, appearing while changing the magnetic field or the charge-carrier density, are due to localization. Neither the original Laughlin paper nor the $T K N_{2}$ one however could give a description of both properties in the same model. Developing a mathematical framework able to reconcile topological and localization properties at once was a challenging problem. Attempts were made by Avron et al. [8] who exhibited quantization but were not able to prove that these quantum numbers were insensitive to disorder. In 1986, H. Kunz [75] went further on and managed to prove this for disorder small enough to avoid filling the gaps between Landau levels.

However Bellissard $[13,14,15]$ proposed to use the Non-Commutative Geometry of Connes [41] to extend the $T K N_{2}$ argument to the case of arbitrary magnetic field and disordered crystal. It turned out that the condition under which plateaus occur was precisely the finiteness of the localization length near the Fermi level. This work was rephrased later on by Avron et al. [9] in terms of charge transport and relative index, filling the remaining gap between experimental observations, theoretical intuition and the mathematical framework. The part concerning the localization was later reconsidered by Aizenman and Graf [3] by using more conventional tools. This Section is devoted to a review of this work which can be found in an extended paper in [22] and in a shorter version in [17]. 


\subsection{Physics}

The Hall effect is observed only for very thin flat conductors. Ideally it is a $2 D$ effect. The thinest possible type of material available at the time of Hall were gold leaves that could be produced with thickness of few micrometers. Nowadays, using potential interface between two semiconductors, it is possible to make the electron gas exactly two-dimensional by forcing the quantized transverse motion to its lower energy state. Let then such a conductor be placed in a perpendicular uniform magnetic field (see Fig. 17)

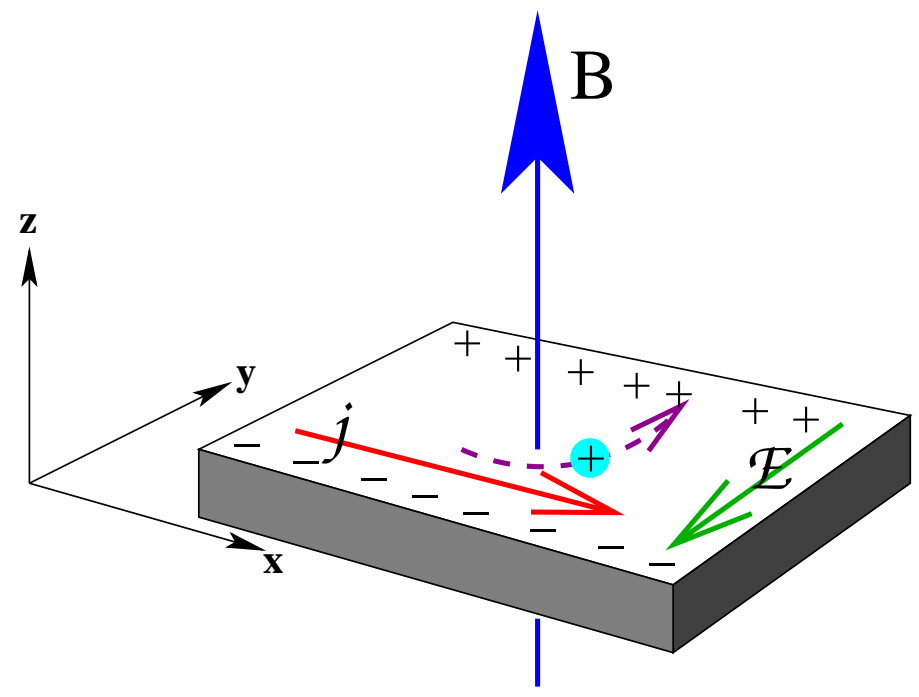

Figure 17: The classical Hall effect

If a constant current $\vec{j}$ is forced in the $x$ direction, the electron fluid will be submitted to the Lorentz force perpendicular to the current and the magnetic field creating an electric field $\overrightarrow{\mathcal{E}}$ along the $y$ axis. In a stationary state, the total force acting on the charge

$$
\vec{F}_{t o t}=q \overrightarrow{\mathcal{E}}+\vec{j} \times \vec{B}, \quad q= \pm e,
$$

vanishes leading to the relation $\vec{j}=\boldsymbol{\sigma} \overrightarrow{\mathcal{E}}$ with $\boldsymbol{\sigma}$, called the conductivity tensor, is an antidiagonal antisymmetric $2 \times 2$ matrix with element $\pm \sigma_{H}$ given by

$$
\sigma_{H}=\nu \frac{e^{2}}{h}, \quad \nu=\frac{n h}{e B} .
$$

Here $n$ is the two-dimensional density of charge carrier, $h$ is Planck's constant, $e$ is the electron charge and $\nu$ is called the filling factor. We remark that the sign of $\sigma_{H}$ depends upon the sign of the carrier charge. In particular, the orientation of the Hall field will change when passing from electrons to holes. This observation, made already by Hall himself in 1880, was understood only in the late twenties after the work by Sommerfeld 
on electron theory [64]. It is commonly used nowadays to determine which kind of particle carries the current.
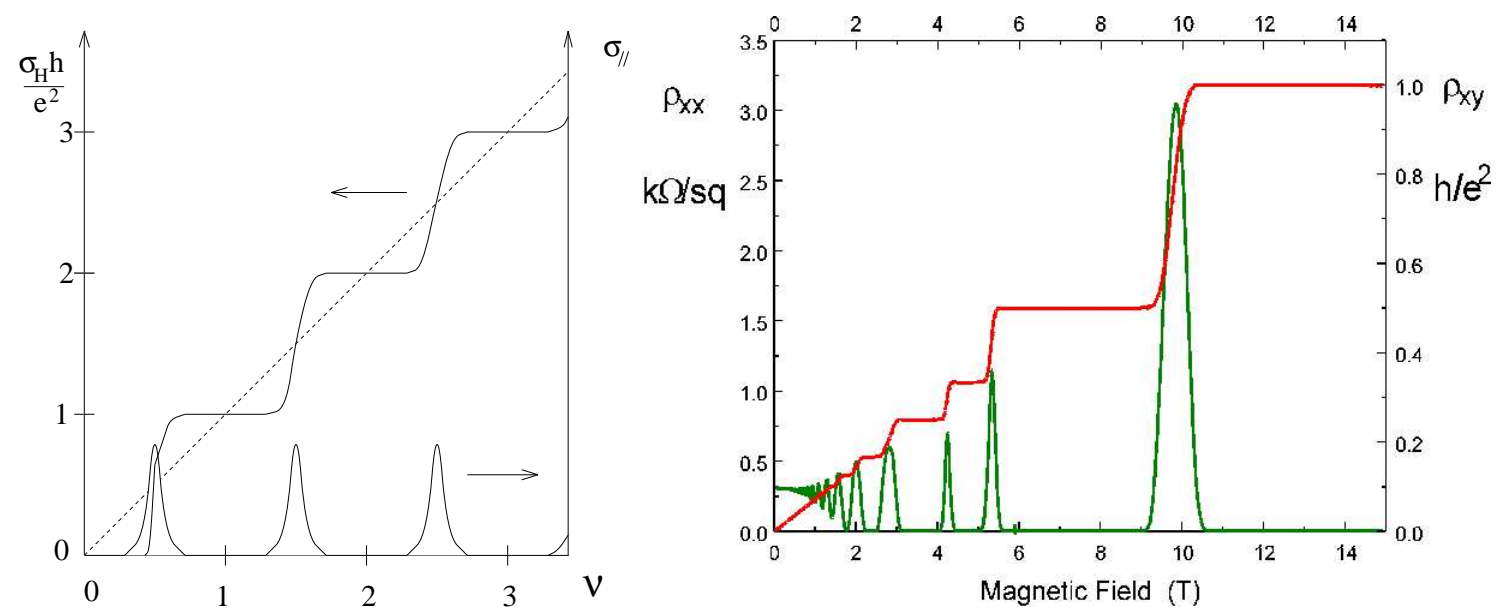

Figure 18: IQHE. left: conductivity (schematic view); right: resistivity (experiment)

The quantity $R_{H}=h / e^{2}$ is called the Hall resistance. It is a universal constant with value $R_{H}=25812.80 \Omega$. $\quad R_{H}$ can be measured directly with an accuracy better than $10^{-8}$ in QHE experiments. Since January 1990, this is the new standard of resistance at the national bureau of standards [106]. As the temperature is lowered to few Kelvins, however, the observation made of semiconductors, like MOSFET [73] or heterojunctions [112], leads to a completely different scenario. As shown in Fig. 18, the conductivity, expressed in units of the Hall resistance, is no longer equal to the filling factor, but is rather a stairlike function with plateaus at integer valued. In heterojunctions, it is even possible to observe plateaus for fractional values of the filing factors (see Fig. 19). The relative accuracy $\delta \sigma_{H} / \sigma_{H}$ of the Hall plateaus can be as low as $10^{-8}-10^{-10}$ depending upon the sample used for the measurement for the integer values. It goes up to $10^{-4}$ for the fraction $1 / 3$ and higher for other observed fractions. The experiments shows also that the direct conductivity and the direct resistivity as well, vanish on the plateaus and are appreciable only for values of the filling factor corresponding to transition between plateaus. That both the direct resistivity and conductivity vanish at the same time is due to the matrix character of the conductivity tensor, the inverse of which being the resistivity tensor. In particular

$$
\boldsymbol{\sigma}=\left(\begin{array}{cc}
0 & \sigma_{H} \\
-\sigma_{H} & 0
\end{array}\right) \quad \Rightarrow \quad \boldsymbol{\rho}=\boldsymbol{\sigma}^{-1}=\left(\begin{array}{cc}
0 & -\sigma_{H}^{-1} \\
\sigma_{H}^{-1} & 0
\end{array}\right)
$$

This experimental fact is explained in term of Anderson localization. Namely for the corresponding the $2 D$ electron gas is submitted to a random potential created by the impurities 


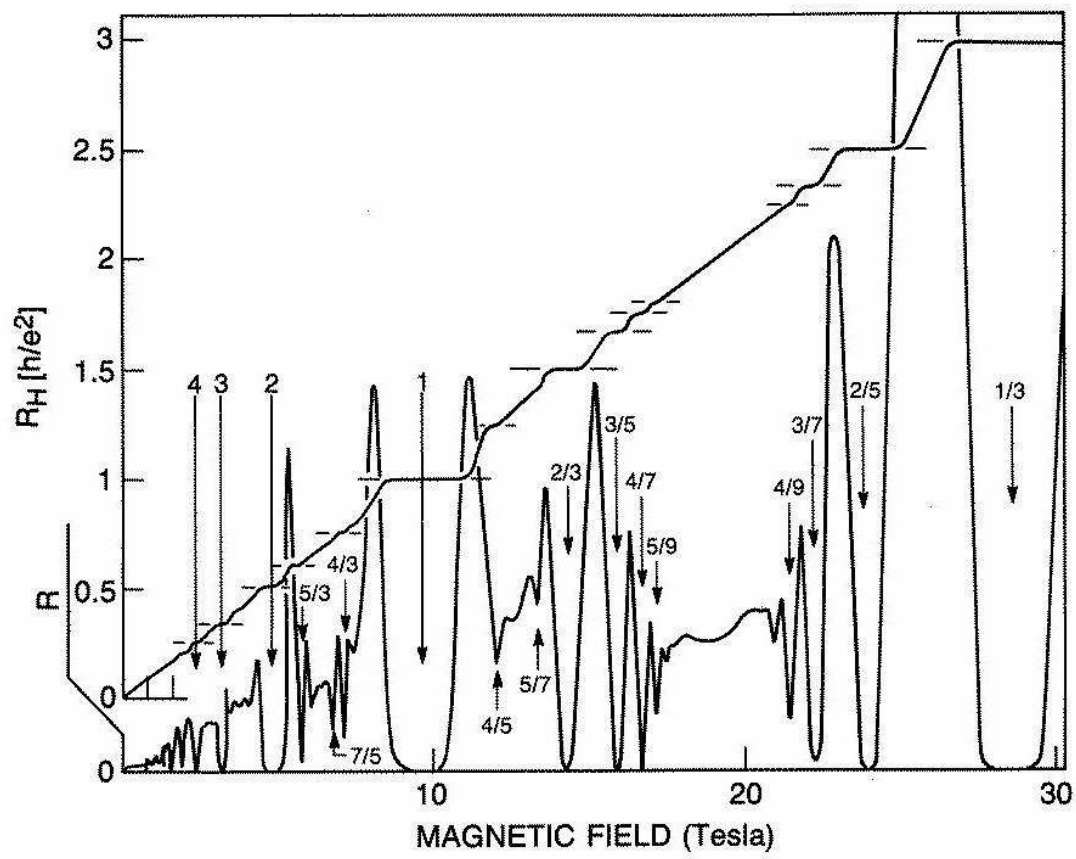

Figure 19: FQHE: fractional plateaus of the resistivity (taken from [116])

used for doping the system. Without such a potential, the one-particle Hamiltonian would be the Landau model of the free electron in a uniform magnetic field [77]

$$
H_{L}=\frac{(\vec{P}-q \vec{A})^{2}}{2 m_{*}},
$$

were $q= \pm e$ is the charge of the carrier, $m_{*}$ its effective mass, $\vec{P}$ is its momentum operator while $\vec{A}=\left(A_{1}, A_{2}\right)$ is the vector potential defined by $\partial_{1} A_{2}-\partial_{2} A_{1}=B$. It is well known that the spectrum of $H_{L}$ is given by the Landau levels

$$
E_{n}=\hbar \omega_{c}\left(n+\frac{1}{2}\right), \quad \omega_{c}=\frac{q B}{m} \quad n=0,1,2, \cdots
$$

Each of these levels has an infinite degeneracy in the infinite volume limit, corresponding to a degeneracy per unit area equal to $g=e B / h$. This number can be intuitively computed as follows: the total number of states available in a large surface of area $S$ is $\Phi / S \phi_{0}$ if $\Phi=B S$ is the total flux through this surface, while $\phi_{o}=h / e$ is the flux quantum. Hence the filling factor is the ratio $\nu=n / g$ of the actual number of electrons et the number of available states in one Landau level. Thus it gives the fraction of the Landau levels that is filled by electrons. 


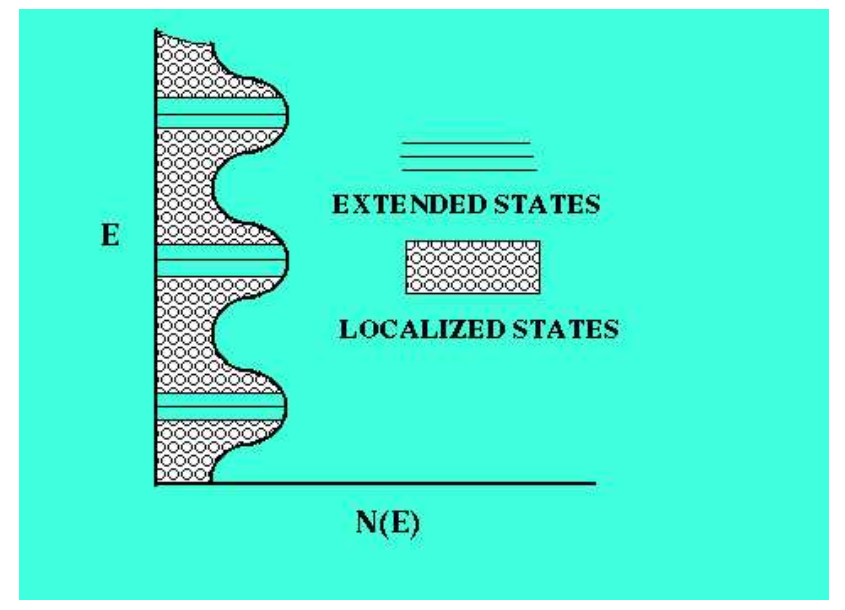

Figure 20: Schematic view of the DOS in the quantum Hall regime

When the disorder potential is turned on, the Landau levels split into large overlaping bands. This gives a density of states (DOS) of the form schematically given in Fig. 20. It shows that the spectrum has no gap. But away from the Landau levels, the states are localized. This has been proved rigorously both for the lattice models [20] and the Landau model with disorder $[10,113]$. Actually, from the renormalization group technics [1] it is expected that all states are localized in the infinite volume limit, but that the localization length diverges at the Landau levels [34]. Since the electron gas obeys Fermi's statistics, at zero temperature all states of lowest energy are occupied up to a maximum value $E_{F}$ called the Fermi level. It is possible to vary the relative position of $E_{F}$ either by changing the charge carrier density $n$, or by modifying the magnetic field $B$. Since both parameters arises through the filling factor, changing $\nu$ is equivalent to change $E_{F}$. Since, in addition there is no spectral gap, the relation $E_{F}=f(\nu)$ is monotonous. Hence, as long as $E_{F}$ stays in a region of localized states, the current cannot change, explaining why there are plateaux of the conductivity. This explanation must be supplemented by a more serious mathematical proof which is explained in the following Sections. However, this argument does not explain why the plateaus occur precisely at the integers. This is related to a topological invariant that cannot be expressed in term of usual Geometry, but which is the noncommutative analog of a Chern number.

\subsection{The Chern-Kubo formula}

Since the seminal paper by Laughlin [79], it is generally accepted that the fractional quantum Hall effect (FQHE) is due to interactions between the charge carriers, creating a new type of groundstate. It is also clear from the experimental observations, that interactions can more or less be ignored for Landau level with quantum numbers $n>2$. 
Hence, if the Coulomb interaction between particles is ignored, the charge carrier fermion fluid is entirely described by the one-particle theory. The quantum motion can be derived from the Hamiltonian of the system. A typical example of one-particle Hamiltonian involved in the QHE for spinless particles, is given by

$$
H_{\omega}=\frac{(\vec{P}-q \vec{A})^{2}}{2 m_{*}}+V_{\omega}(\vec{x}),
$$

where $V_{\omega}(\vec{x})$ describes the potential created by disorder in the Hall bar. Here $\omega$, which denotes the configuration of disorder, can be seen as a point of the Hull $\Omega$ associated with the sublattice of impurities. Then the covariance condition $V_{\omega}(\vec{x}-\vec{a})=V_{T^{\vec{a}} \omega}(\vec{x})$ expresses that moving the sample or changing the reference axis backward are equivalent. Such a model is typical, and is actually accurate for semiconductors. But it may be replaced by others, such as lattice approximants, or particle with spin. In any case, the oneparticle Hamiltonian describing the fermion fluid becomes affiliated to (see Definition 7) the $C^{*}$-algebra $\mathcal{A}=\mathcal{C}\left(\Omega \rtimes \mathbb{R}^{2}, B\right)$ (see Definition 6 ).

Standard results in transport theory permit to compute the conductivity in term of the linear response of the fermion fluid under the influence of an external field. This is the famous Green-Kubo formula. In the QHE-limit, namely in the limit of (i) zero temperature, (ii) infinite sample size, (iii) negligible collision processes, (iv) vanishingly small electric fields, the direct conductivity either vanishes or is infinite, whereas the transverse conductivity, when defined, is given by $[110,22]$

$$
\sigma_{H}=\frac{e^{2}}{h} \mathbf{C h}\left(P_{F}\right)=\frac{e^{2}}{h} 2 \imath \pi \mathcal{T}_{\mathbb{P}}\left(P_{F}\left[\partial_{1} P_{F}, \partial_{2} P_{F}\right]\right) .
$$

It turns out that $\mathbf{C h}$ is nothing but the non commutative analog of a Chern character. Thus Kubo's formula gives rise to a Chern character in the QHE limit. This is why eq (38) can be called the Kubo-Chern formula, associating Japan with China. The main properties of the non commutative Chern character are the following [41]

(i) homotopy invariance: given two equivalent $\mathcal{C}^{1}$ projections $P$ and $Q$ in $\mathcal{A}$, namely such that there is $U \in \mathcal{C}^{1}(\mathcal{A})$ with $P=U^{*} U$ and $Q=U U^{*}$, then $\mathbf{C h}(P)=\mathbf{C h}(Q)$. This is actually what happens if $P$ and $Q$ are homotopic in $\mathcal{C}^{1}(\mathcal{A})$.

(ii) additivity: given two $\mathcal{C}^{1}$ orthogonal projections $P$ and $Q$ in $\mathcal{A}$, namely such that $P Q=Q P=0$ then $\mathbf{C h}(P \oplus Q)=\mathbf{C h}(P)+\mathbf{C h}(Q)$.

In particular, the homotopy invariance shows that $\mathbf{C h}\left(P_{F}\right)$, when it is defined, is a topological quantum number. One of the main results of Noncommutative Geometry is that this Chern character is an integer provide it is well defined. Thus, thanks to eq. (38) the Hall conductance is quantized. It will be shown in Section 5.4 that this Chern character is well defined precisely whenever the Fermi level lies in a region of localized states. Moreover changing the value of the filling factor produces the moving of the Fermi level, which does not change the Chern character as long as the localization length stay bounded. 


\subsection{The Four Traces Way}

In this section four different traces will be defined and used. They are technically needed to express the complete results of this theory. The first one is the usual trace $\mathrm{Tr}$ on matrices or on trace-class operators. The second one, introduced in Section 2.6, eq. (17), is the trace per unit volume $\mathcal{T}_{\mathbb{P}}$ attached to an $\mathbb{R}^{2}$-invariant probability measure $\mathbb{P}$ on the Hull. The third one $\operatorname{Tr}_{S}$ is the graded trace or supertrace introduced in this Section below. This is the first technical tool proposed by A. Connes $[38,41]$ to define the cyclic cohomology and constitutes the first important step in proving quantization of the Hall conductance [14]. The last one is the Dixmier trace $\operatorname{Tr}_{\text {Dix }}$ defined by Dixmier in 1964 [45] and of which the importance for Quantum Differential Calculus was emphasized by A. Connes [39, 40, 41]. It will be used in connection with Anderson's localization.

Let $\mathcal{H}$ be the physical one-particle Hilbert space of Section 2.6 namely $L^{2}\left(\mathbb{R}^{2}\right)$. In the language of Noncommutative Geometry, $\mathcal{H}$ can be seen as the space of sections of a hermitian vector bundle over the noncommutative Brillouin zone. Following Atiyah's proof of the Index theorem, through the Dirac operator [7], it is convenient to introduced a spin bundle (a similar construction has been proposed in Section 2.6). Practically, here, this is done through the new Hilbert space $\hat{\mathcal{H}}=\mathcal{H}_{+} \oplus \mathcal{H}_{-}$with $\mathcal{H}_{ \pm}=\mathcal{H}$. A grading operator $\widehat{G}$ and the (longitudinal) Dirac operator $D$ are defined as follows:

$$
\widehat{G}=\left(\begin{array}{cc}
+1 & 0 \\
0 & -1
\end{array}\right), \quad D=\left(\begin{array}{cc}
0 & X \\
\bar{X} & 0
\end{array}\right), \quad F=\left(\begin{array}{cc}
0 & \frac{X}{|X|} \\
\frac{X}{|X|} & 0
\end{array}\right),
$$

where $X=X_{1}+\imath X_{2}$ (here the dimension is $d=2$ ). It is clear that $D$ is selfadjoint and satisfies $D^{2}=X^{2} \mathbf{1}$. Moreover $F$ is the phase of $D$, namely $F=D|D|^{-1}$. Then $F=F^{*}$ and $F^{2}=1$. A bounded operator $T$ acting on $\hat{\mathcal{H}}$ will said to have degree 0 if it commutes with $\widehat{G}$ and of degree 1 if it anticommutes with $\widehat{G}$. The graded commutator (or supercommutator) of two operators and the graded differential $d T$ are defined by

$$
\left[T, T^{\prime}\right]_{S}=T T^{\prime}-(-)^{\operatorname{deg}(T) \operatorname{deg}\left(T^{\prime}\right)} T^{\prime} T, \quad d T=[F, T]_{S} .
$$

Then, $d^{2} T=0$. The graded trace $\operatorname{Tr}_{S}$ (or supertrace) is defined by

$$
\operatorname{Tr}_{S}(T)=\frac{1}{2} \operatorname{Tr}_{\hat{\mathcal{H}}}\left(\widehat{G} F[F, T]_{S}\right)=\operatorname{Tr}_{\mathcal{H}}\left(T_{++}-u T_{--} \bar{u}\right),
$$

where $u=X /|X|$ and $T_{++}$and $T_{--}$are the diagonal components of $T$ with respect to the decomposition of $\hat{\mathcal{H}}$. It is a linear map on the algebra of operators such that $\operatorname{Tr}_{S}\left(T T^{\prime}\right)=\operatorname{Tr}_{S}\left(T^{\prime} T\right)$. However, this trace is not positive. Observables in $\mathcal{A}$ will become operators of degree 0 , namely $A \in \mathcal{A}$ will be represented by $\hat{A}_{\omega}=A_{\omega} \oplus A_{\omega}$.

Given a Hilbert space $\mathcal{H}$, the characteristic values $\mu_{1}, \ldots, \mu_{n}, \ldots$ of a compact operator $T$ are the eigenvalues of $|T|=\left(T T^{*}\right)^{1 / 2}$ labeled in the decreasing order. The Mačaev ideals $\mathcal{L}^{p+}(\mathcal{H})$ is the set of compact operators on $\mathcal{H}$ with characteritic values satisfying 


$$
\|T\|_{p+}=\sup _{N \rightarrow \infty} \frac{1}{\ln N} \sum_{n=1}^{N} \mu_{n}^{p}<\infty .
$$

Let Lim be a positive linear functional on the space of bounded sequences $l_{+}^{\infty}(\mathbf{N})$ of positive real numbers which is translation and scale invariant. For $T \in \mathcal{L}^{1+}(\mathcal{H})$ its Dixmier trace is defined by

$$
\operatorname{Tr}_{\text {Dix }}(T)=\operatorname{Lim}\left(\frac{1}{\ln N} \sum_{n=1}^{N} \mu_{n}\right)
$$

Clearly, $T \in \mathcal{L}^{1+}$ if and only if $\operatorname{Tr}_{\text {Dix }}(|T|)<\infty$ and if the sequence $\left(\frac{1}{\ln N} \sum_{n=1}^{N} \mu_{n}\right)$ converges, then all functionals Lim of the sequence are equal to the limit and the Dixmier trace is given by this limit. From this definition, it can be shown that $\operatorname{Tr}_{\mathrm{Dix}}$ is a trace $[45,41]$.

The first important result is provided by a formula that was suggested by a result of A. Connes [39]. Namely if $A \in \mathcal{C}^{1}(\mathcal{A})$ and if $\vec{\nabla}=\left(\partial_{1}, \partial_{2}\right)[22]$ :

$$
\mathcal{T}_{\mathbb{P}}\left(|\vec{\nabla} A|^{2}\right)=\frac{1}{\pi} \operatorname{Tr}_{\text {Dix }}\left(\left|d A_{\omega}\right|^{2}\right), \quad \text { for } \mathbb{P} \text {-almost all } \omega
$$

Let now $\mathcal{S}$ denote the closure of $\mathcal{C}^{1}(\mathcal{A})$ under the noncommutative Sobolev norm $\|A\|_{\mathcal{S}}^{2}=$ $\mathcal{T}_{\mathbb{P}}\left(A^{*} A\right)+\mathcal{T}_{\mathbb{P}}\left(\vec{\nabla} A^{*} \vec{\nabla} A\right)$. The eq. (41) shows that for any element $A \in \mathcal{S}, d A_{\omega}$ belongs to $\mathcal{L}^{2+}(\hat{\mathcal{H}}), \mathbb{P}$-almost surely. In what follows, $L^{\infty}\left(\mathcal{A}, \mathcal{T}_{\mathbb{P}}\right)$ denotes the weak closure of $\mathcal{A}$ in the GNS-representation with respect to $\mathcal{T}_{\mathbb{P}}$ (see Section 2.6).

The following formula, valid for $A_{0}, A_{1}, A_{2} \in \mathcal{C}^{1}(\mathcal{A})$, is the next important result proved in $[38,14,9,22]$ :

$$
\int_{\Omega} d \mathbb{P}(\omega) \operatorname{Tr}_{S}\left(\hat{A}_{0, \omega} d \hat{A}_{1, \omega} d \hat{A}_{2, \omega}\right)=2 \imath \pi \mathcal{T}_{\mathbb{P}}\left(A_{0} \partial_{1} A_{1} \partial_{2} A_{2}-A_{0} \partial_{2} A_{1} \partial_{1} A_{2}\right) .
$$

This formula actually extends to $A_{i} \in L^{\infty}\left(\mathcal{A}, \mathcal{I}_{\mathbb{P}}\right) \cap \mathcal{S}$. For indeed, the right hand side is well defined by the Cauchy-Schwartz inequality. On the other hand, thanks to eq. (41), $d \hat{A}_{i, \omega} \in \mathcal{L}^{2+} \subset \mathcal{L}^{3}(\hat{\mathcal{H}})$, if $\mathcal{L}^{p}(\mathcal{H})$ denotes the Schatten ideal of compact operators $T$ on $\mathcal{H}$ with traceclass $|T|^{p}$. Then the integrand occuring under the integral of the left hand side can be written as $\operatorname{Tr}_{\hat{\mathcal{H}}}\left(\widehat{G} F d \hat{A}_{0, \omega} d \hat{A}_{1, \omega} d \hat{A}_{2, \omega}\right)$ which is well defined, thanks to Hölder's inequality.

Applying these formulæ to the Fermi projection, the Chern character $\mathbf{C h}\left(P_{F}\right)$ is well defined provide $P_{F} \in \mathcal{S}$ and

$$
\mathbf{C h}\left(P_{F}\right)=\int_{\Omega} d \mathbb{P}(\omega) \operatorname{Tr}_{S}\left(\widehat{P}_{F, \omega} d \widehat{P}_{F, \omega} d \widehat{P}_{F, \omega}\right)
$$


The last step is a consequence of the Calderón-Fedosov formula [32, 50] namely the operator $\left.P_{\omega} F^{+-}\right|_{P_{\omega} \mathcal{H}_{-}}$is Fredholm and its index is an integer given by:

$$
n(\omega)=\operatorname{Ind}\left(\left.P_{\omega} F^{+-}\right|_{P_{\omega} \mathcal{H}_{-}}\right)=\operatorname{Tr}_{S}\left(\widehat{P}_{F, \omega} d \widehat{P}_{F, \omega} d \widehat{P}_{F, \omega}\right) .
$$

It remains to show that this index is $\mathbf{P}$-almost surely constant. By the covariance condition $\left.P_{T^{a} \omega} F^{+-}\right|_{P_{T^{a}} \mathcal{H}_{-}}$and $\left.P_{\omega} T(a)^{-1} F^{+-} T(a)\right|_{P_{\omega} \mathcal{H}_{-}}$are unitarily equivalent, so that they have same Fredholm index. Moreover $\left.P_{\omega} T(a)^{-1} F^{+-} T(a)\right|_{P_{\omega} \mathcal{H}_{-}}-\left.P_{\omega} F^{+-}\right|_{P_{\omega} \mathcal{H}_{-}}$is easily seen to be compact so that $\left.P_{T^{a} \omega} F^{+-}\right|_{P_{T^{a}} \mathcal{H}_{-}}$have the same index as $\left.P_{\omega} F^{+-}\right|_{P_{\omega} \mathcal{H}_{-}}$. In other words, $n(\omega)$ is a $\mathbb{R}^{2}$-invariant function of $\omega$. The probability $\mathbb{P}$ being $\mathbb{R}^{2}$-invariant and ergodic, $n(\omega)$ is $\mathbb{P}$-almost surely constant. Consequently, since $F^{+-}=u$, if $P_{F} \in \mathcal{S}$ :

$$
\mathbf{C h}\left(P_{F}\right)=\operatorname{Ind}\left(\left.P_{F, \omega} u\right|_{P_{F, \omega} \mathcal{H}}\right) \in \mathbf{Z}, \quad \mathbf{P} \text {-almost surely . }
$$

In [9], Avron et al. showed that this index can also be interpreted as

$$
\operatorname{Ind}\left(\left.P_{F, \omega} u\right|_{P_{F, \omega} \mathcal{H}}\right)=\operatorname{Tr}_{\mathcal{H}}\left(\left(u P_{F, \omega} u^{-1}-P_{F, \omega}\right)^{3}\right),
$$

The right hand side is called the relative index of $u P_{F, \omega} u^{-1}$ and $P_{F, \omega}$. It represents the variation of the dimension of the projector $P_{F, \omega}$ when the unitary transformation $u$ is applied. It turns out that $u=X /|X|$ is exactly the (singular) gauge transformation applied to the original Hamiltonian whenever an infinitely flux tube is pierced at the origin and the flux is adiabatically increased from 0 to one flux quantum. Laughlin [78] argued that this number is exactly the number of charges that are sent to infinity under this adiabatic transformation.

\subsection{Localization}

It remains to show how the condition $P_{F} \in \mathcal{S}$ is related to the Anderson localization. The easiest way to define the localization length consists in measuring the averaged square displacement of a wave packets on the long run. Let $\Delta \subset \mathbb{R}$ be an interval. Let $P_{\Delta}$ be the eigenprojection of the Hamiltonian corresponding to energies in $\Delta$. If $X$ is the position operator, let $X_{\Delta, \omega}(t)=e^{\frac{2}{\hbar} H_{\omega} t} P_{\Delta, \omega} X P_{\Delta, \omega} e^{-\frac{2}{\hbar} H_{\omega} t}$. The $\Delta$-localization length is defined as:

$$
l^{2}(\Delta)=\limsup _{T \rightarrow \infty} \int_{0}^{T} \frac{d t}{T} \int_{\Omega} d \mathbb{P}(\omega)<0\left|\left(X_{\Delta, \omega}(t)-X_{\Delta, \omega}(0)\right)^{2}\right| 0>.
$$

It is shown in $[22]$ that, equivalently,

$$
\begin{aligned}
l^{2}(\Delta) & =\limsup _{T \rightarrow \infty} \int_{0}^{T} \frac{d t}{T} \mathcal{T}_{\mathbb{P}}\left(\left|\vec{\nabla}\left(e^{-\frac{\imath}{\hbar} H t} P_{\Delta}\right)\right|^{2}\right) \\
& =\sup _{\mathcal{P}} \sum_{\Delta^{\prime} \in \mathcal{P}} \mathcal{T}_{\mathbb{P}}\left(\left|\vec{\nabla} P_{\Delta^{\prime}}\right|^{2}\right) .
\end{aligned}
$$


where $\mathcal{P}$ runs in the set of finite partitions of $\Delta$ by Borel subsets. Moreover [22]

Theorem 25 If $\ell^{2}(\Delta)<\infty$, then the spectrum of $H_{\omega}$ is pure point in $\Delta, \mathbb{P}$-almost surely.

The density of states is the positive measure $\mathcal{N}$ on $\mathbb{R}$ defined by $\int_{\mathbb{R}} d \mathcal{N}(E) f(E)=$ $\mathcal{T}_{\mathbb{P}}(f(H))$ (see eq. $(22)$ ) for $f$ a continuous function with compact support. It turns out [22] that if $l^{2}(\Delta)<\infty$, there is a positive $\mathcal{N}$-square integrable function $\ell$ on $\Delta$ such that

$$
l^{2}\left(\Delta^{\prime}\right)=\int_{\Delta^{\prime}} d \mathcal{N}(E) l(E)^{2}
$$

for any subinterval $\Delta^{\prime}$ of $\Delta$. Then, $\ell(E)$ is the localization length at energy $E$.

Thanks to eq. (46), (47) the finiteness of the localization length in the interval $\Delta$ implies that $[22]$

(i) $P_{F} \in \mathcal{S}$ whenever the Fermi level $E_{F}$ lies in $\Delta$,

(ii) $E_{F} \in \Delta \mapsto P_{F} \in \mathcal{S}$ is continuous (for the Sobolev norm) at every regularity point of $\mathcal{N}$

(iii) $\mathbf{C h}\left(P_{F}\right)$ is constant on $\Delta$, leading to existence of plateaus for the transverse conductivity.

(iv) If the Hamiltonian is changed continuously (in the norm resolvent topology), $\mathbf{C h}\left(P_{F}\right)$ stay constant as long as the localization length remains finite at the Fermi level.

As a Corollary, between two Hall plateaus with different indices, the localization length must diverge [59, 75, 34]. The reader will find in [22] how to compute practically the Hall index using homotopy (property (iv)) and explicit calculation for simple models. 


\section{References}

[1] E. Abrahams, P.W. Anderson, D.C. Licciardello, T.V. Ramakrishnan, Scaling Theory of Localization: Absence of Quantum Diffusion in Two Dimensions, Phys. Rev. Lett., 42, 673-676, (1979).

[2] J.F. Adams, On Chern characters and the structure of the unitary group., Proc. Cambridge Philos. Soc., 57, 189-199, (1961).

[3] M. Aizenman, G.M. Graf, Localization bounds for an electron gas, J. Phys. A, 31, 6783-6806, (1998).

[4] J. Anderson, I.F. Putnam, Topological invariants for substitution tilings and their $C^{*}$-algebras, Ergodic Th. and Dynam. Sys., 18, 509-537, (1998).

[5] N. W. Ashcroft, N. D. Mermin, Solid State Physics, Holt, Rinehart and Winston Eds., (1976).

[6] M. F. Atiyah, F. Hirzebruch, Vector bundles and homogeneous spaces, Proc. Symp. Pure Math., 3, 7-38, (1961).

[7] M.F. Atiyah, K-theory, Lecture notes by D. W. Anderson, W. A. Benjamin, Inc., New York-Amsterdam (1967).

[8] J.E. Avron, R. Seiler, B. Simon, Homotopy and quantization in condensed matter physics, Phys. Rev. Lett., 51, 51-53, (1983).

[9] J.E. Avron, R. Seiler, B. Simon, Charge deficiency, charge transport and comparison of dimensions, Commun. Math. Phys., 159, 399-422, (1994).

[10] J.-M. Barbaroux, J.-M. Combes, P.D. Hislop, Landau Hamiltonians with unbounded random potentials, Lett. Math. Phys., 40, 355-369, (1997).

[11] C. Beeli, T. Gödecke, R. Lück, Highly faceted growth shape of microvoids in icosahedral Al-Mn-Pd, Philos. Mag. Lett., 78, 339-48, (1998).

[12] J. Bellissard, Schrödinger's operators with an almost periodic potential : an overview, in Lecture Notes in Phys., 153, Springer Verlag, Berlin Heidelberg, New York, (1982).

[13] J. Bellissard, K-Theory of $C^{*}$-algebras in Solid State Physics, in Statistical Mechanics and Field Theory, Mathematical Aspects, T.C. Dorlas, M. N. Hugenholtz \& M. Winnink Eds., Lecture Notes in Physics, 257, 99-156, (1986). 
[14] J. Bellissard, Ordinary Quantum Hall Effect and Non Commutative Differential Geometry, in Localization in Disordered Systems, edited by Ziesche \& Weller, TeubnerVerlag, Leipzig, (1987).

[15] J. Bellissard, $C^{*}$ algebras in solid state physics. $2 D$ electrons in a uniform magnetic field., in Operator algebras and applications, Vol. 2, 49-76, London Math. Soc. Lecture Note Ser., 136, Cambridge Univ. Press, Cambridge, (1988).

[16] J. Bellissard, Gap Labelling Theorems for Schrödinger's Operators, in From Number Theory to Physics, pp. 538-630, Les Houches March 89, Springer, J.M. Luck, P. Moussa \& M. Waldschmidt Eds., (1993).

[17] J. Bellissard, Non-Commutative Geometry and Quantum Hall Effect, in the Proceedings of the International Conference of Mathematic, (Zürich 94), Birkhäuser (1995).

[18] J. Bellissard, Coherent and dissipative transport in aperiodic solids, to appear in Proceeding 38th Winter School of Theoretical Physics, Ladek, Poland, 6-15 Feb 2002; see http://www.math.gatech.edu/ jeanbel/ (publications)..

[19] J. Bellissard, D. Testard, Quasi Periodic Hamiltonians : a Mathematical Approach, in Operator Algebras and Applications, Coll. AMS, Vol.2, pp. 579, (1982).

[20] J. Bellissard, D.R. Grempel, F. Martinelli, E. Scoppola, Localization of electrons with spin-orbit or magnetic interactions in a two-dimensional disordered crystal, Phys. Rev. B, 33, 641-644, (1986)

[21] J. Bellissard, A. Bovier, J.M. Ghez, Spectral Properties of a tight binding Hamiltonian with Period Doubling Potential, Comm. Math. Phys., 135, 379-399, (1991).

[22] J. Bellissard, H. Schulz-Baldes, A. van Elst, The Non Commutative Geometry of the Quantum Hall Effect, J. Math. Phys., 35, 5373-5471, (1994).

[23] J. Bellissard, D. Hermmann, M. Zarrouati, Hull of Aperiodic Solids and Gap Labelling Theorems, in Directions in Mathematical Quasicrystals, CRM Monograph Series, Volume 13, (2000), 207-259, M.B. Baake \& R.V. Moody Eds., AMS Providence.

[24] J. Bellissard, R. Benedetti, J.-M. Gambaudo, Spaces of Tilings, Finite Telescopic Approximations and Gap-labelling, math.DS/0109062, submitted to Comm. Math. Phys., (2002).

[25] M. Benameur, H. Oyono-Oyono, Gap-labelling for quasi-crystals (proving a conjecture by J. Bellissard), math.KT/0112113 (2001).

[26] B. Blackadar, K-Theory for Operator Algebras, Springer, New York (1986); 2nd ed., Cambridge University Press, Cambridge (1998). 
[27] F. Bloch, Über die Quantenmechanik der Elektronen in Kristallgittern, Z. Phys., 52, 555-600, (1928).

[28] M. Born, T. von Kármán, Über Schwingungen in Raumgitter, Phys. Zs., 13, 297-309 (1912).

[29] L. P. Bouckaert, R. Smoluchowski, E. Wigner, Theory of Brillouin zones and symmetry of wave functions in crystals, Phys. Rev., 50, 58-67, (1936).

[30] L. Brillouin, Les électrons libres dans les métaux et le rôle des reflexions de Bragg, J. Phys. Radium,1, 377-400, (1930).

[31] N. de Bruijn, Sequences of zeroes and ones generated by special production rules, Kon. Neder. Akad. Wetensch. Proc., A 84, 27-37, (1981); Algebraic theory of Penrose's non-periodic tilings of the plane, Kon. Neder. Akad. Wetensch. Proc., A 84, 39-66, (1981).

[32] A.-P. Calderón, The analytic calculation of the index of elliptic equations, Proc. Nat. Acad. Sci. U.S.A., 57, 1193-1194, (1967).

[33] R. Carmona, J. Lacroix, Random Schrödinger Operators, Birkhaüser, Basel (1990).

[34] J.T. Chalker, P.D. Coddington, Percolation, quantum tunnelling and the integer Hall effect, J. Phys. C, 21, 2665-2679, (1988).

[35] F.H. Claro, W.H. Wannier, Closure of bands for Bloch electrons in a magnetic field., Phys. Status Sol. B, 88, K147-151, (1978).

[36] A. Connes, Sur la théorie non commutative de l'intégration, in Algèbres d'Opérateurs, Lecture Notes in Mathematics, 725, pp. 19-143, Springer, Berlin (1979).

[37] A. Connes, An analog of the Thom isomorphism for crossed products of a $C^{*}$-algebra by an action of $\mathbb{R}$, Adv. Math., 39, 31-55, (1981).

[38] A. Connes, Non-commutative differential geometry, Publ. I.H.É.S., 62, 257-360, (1986).

[39] A. Connes, The action functional in non-commutative geometry, Commun. Math. Phys., 117, 673-683, (1988).

[40] A. Connes, Trace de Dixmier, modules de Fredholm et géométrie riemannienne, in Conformal field theories and related topics, (Annecy-le-Vieux, 1988), Nuclear Phys. B Proc. Suppl., 5B, 65-70, (1988).

[41] A. Connes, Noncommutative Geometry, Acad. Press., San Diego (1994). 
[42] I. Cornfeld, S. Fomin, Ya. G. Sinai, Ergodic Theory, Springer-Verlag, New York, (1982).

[43] H. L. Cycon, R. G. Froese, W. Kirsch, B. Simon, Schrödinger operators, with application to quantum mechanics and global geometry, (Springer Study ed.), Texts and Monographs in Physics. Berlin etc., Springer-Verlag, (1987).

[44] J. Dixmier, Existence de traces non normales, Comptes Rendus Acad. Sc. Paris, T. 262, no. 20, 1107-1108 (1966).

[45] J. Dixmier, Les algèbres d'opérateurs dans l'espace hilbertien (Algèbres de von Neumann), Gauthier-Villars, Paris (1957); Les $C^{*}$-algèbres et leurs représentations, Deuxième édition, Gauthier-Villars Éditeur, Paris (1969).

[46] M. Duneau and A. Katz, Quasiperiodic patterns, Phys. Rev. Lett.. 54, 2688-2691, (1985).

[47] M. Duneau and A. Katz, Quasiperiodic patterns and icosahedral symmetry, J. Phys. (France), 47, 181-196 (1986).

[48] A. Einstein, Die Plancksche Theorie der Strahlung und die Theorie der spezifischen Wärme, Ann. Phys., 22, 180-190 (1907).

[49] V. Elser, Indexing problem in quasicrystal diffraction, Phys. Rev., B 32, 4892-4898, (1985); and: Comment on "Quasicrystals: a new class of ordered structures", Phys. Rev. Lett., 54, 1730, (1985).

[50] B. Fedosov, A direct proof of the formula for the index of an elliptic system in Euclidean space, Functional Anal. App., 4, 339-341, (1970).

[51] H. Feshbach, A unified theory of nuclear reactions. I, Ann. Phys., 5, 357-390 (1958); A unified theory of nuclear reactions. II, Ann. Phys., 19, 287-313 (1962).

[52] I.R. Fisher, Z. Islam, A.F. Panchula, K.O. Cheon, M.J. Kramer, P.C. Canfield, A.I. Goldman, Growth of large-grain $R-M g-Z n$ quasicrystals from the ternary melt (R=Y, Er, Ho, Dy, Tb), Philos. Mag. B, 77, 1601-15, (1998).

[53] A. H. Forrest and J. R. Hunton, The cohomology and K-theory of commuting homeomorphisms of the Cantor set, Erg. Th. ES Dyn. Syst., 19, 611-625, (1999).

[54] A. H. Forrest, J. R. Hunton, J. Kellendonk, Projection quasicrystal I: toral rotations, preprint (1998), NTNU, Trondheim; and: Projection quasicrystal II: versus substitution tilings, preprint No. 396 SFB-288, TU Berlin (1999).

[55] V. Georgescu, A. Iftimovici, Crossed Product of $C^{*}$-algebras and Spectral Analysis of Quantum Hamiltonians, preprint 2002, to be published in Commun. Math. Phys.. 
[56] C. Gérard, A. Martinez, J. Sjöstrand, A mathematical approach to the effective Hamiltonian in perturbed periodic problems, Comm. Math. Phys., 142, 217-244, (1991).

[57] V. V. Grushin, On a class of elliptic pseudodifferential operators degenerate on a submanifold, Math. USSR, Sbornik, 13, 155-185, (1971).

[58] E. Hall, On a new action of the magnet on electric currents, Amer. J. Math., 2, 287 (1879); and in Quantum Hall effect: a perspective, edited by A. Mac Donald, Kluwer Academic Publishers, Dordrecht, (1989).

[59] B.I. Halperin, Quantized Hall conductance, current-carrying edge states and the existence of extended states in a two-dimensional disordered potential, Phys. Rev. B, 25, 2185-2190, (1982).

[60] P.G. Harper, (i) Single Band Motion of Conduction Electrons in a Uniform Magnetic Field, Proc. Phys. Soc. Lond., A68, 874-878, (1955);(ii) The General Motion of Conduction Electrons in a Uniform Magnetic Field, with Application to the Diamagnetism of Metals, Proc. Phys. Soc. Lond., A68, 879-892, (1955).

[61] B. Helffer, J. Sjöstrand, Opérateurs de Schrödinger avec champs magnétiques faibles et constants, Séminaire sur les Équations aux Dérivées Partielles, 1988-1989, Exp. No. XII, École Polytech., Palaiseau, (1989).

[62] F. Hippert \& D. Gratias Eds., Lectures on Quasicrystals, Editions de Physique, Les Ulis, (1994).

[63] D.R. Hofstadter, Energy levels and wave functions of Bloch electrons in a rational or irrational magnetic field, Phys. Rev. B14, 2239-2249, (1976).

[64] Lillian Hoddeson (Editor), Ernest Braun, Jurgen Teichmann, Spencer Weart (Editor), Frederick Seitz, Out of the Crystal Maze : Chapters from the History of Solid State Physics, Oxford Univ Press, (1992).

[65] H. Jones, The Theory of Brillouin Zones and Electronic States in Crystals, North Holland, Amsterdam (1960).

[66] R. Joynt, R. Prange, Conditions for the quantum Hall effect, Phys. Rev. B, 29, 3303-3317, (1984).

[67] P. A. Kalugin, A. Y. Kitaev, L. S. Levitov, $A l_{0.86} M n_{0.14}$ : a six-dimensional crystal, JETP Lett., 41, 145-149, (1985); and: 6-dimensional properties of $A l_{0.86} M n_{0.14 .}, \mathrm{J}$. Phys. Lett. (France), 46, L601-L607, (1985).

[68] J. Kaminker, I.F. Putnam, A proof of the Gap Labeling Conjecture, in arXiv math.KT/0205102. 
[69] A. Katok, B. Hassenblatt, Introduction to the Modern Theory of Dynamical Systems, Cambridge University Press, (1995).

[70] J. Kellendonk, I. F. Putnam, Tiling $C^{*}$-algebras and $K$-theory, in Directions in Mathematical Quasicrystals, CRM Monograph Series, Volume 13, (2000), 177-206, M.B. Baake \& R.V. Moody Eds., AMS Providence.

[71] J. L. Kelley, General Topology, Springer, New-York Heidelberg Berlin, reprint (1975).

[72] (i) M. Kléman and J.-F. Sadoc, J. Phys. (Paris) Lett., 40, L569 (1979); (ii) J.F. Sadoc, J. Non-Cryst. Solids 44, 1 (1981); (iii) R. Mosseri, D.P. DiVincenzo, J.F. Sadoc, M.H. Brodsky, Polytope model and the electronic and structural properties of amorphous, Phys. Rev., B32, 3974-4000, (1985).

[73] K. v. Klitzing, G. Dorda, M. Pepper, New method for high accuracy determination of the fine structure constant based on quantized Hall resistance, Phys. Rev. Lett., 45, 494-497, (1980).

[74] P. Kramer and R. Neri, On periodic and non-periodic space fillings of $E^{m}$ obtained by projections, Acta. Cryst., A 40, 580-587, (1984); and: Acta. Cryst., A 41, 619, (1985) (Erratum).

[75] H. Kunz, The quantized Hall effect for electrons in a random potential, Commun. Math. Phys., 112, 121-145, (1987).

[76] J. C. Lagarias, P. A. B. Pleasants, Repetitive Delone sets and perfect quasicrystals, in math.DS/9909033, (1999).

[77] L.D. Landau, Diamagnetismus der Metalle, Z. für Phys., 64, 629-637, (1930).

[78] R.B. Laughlin, Quantized Hall conductivity in two-dimension, Phys. Rev. B, 23, 5632-5633, (1981).

[79] R.B. Laughlin, Anomalous Quantum Hall Effect: An Incompressible Quantum Fluid with Fractionally Charged Excitations, Phys. Rev. Lett., 50, 1395-1398, (1983)

[80] T. T. Q. Le, Local rules for quasiperiodic tilings, in: The Mathematics of Long-Range Aperiodic Order, NATO ASI Series C 489, Kluwer, Dordrecht (1997), pp. 331-366.

[81] D. Levine and P. J. Steinhardt, Quasicrystals: a new class of ordered structures, Phys. Rev. Lett., 53, 2477-2480, (1984).

[82] Y. Meyer, Algebraic Numbers and Harmonic Analysis, North-Holland, Amsterdam (1972); and: Quasicrystals, Diophantine approximation and algebraic numbers, in: Beyond Quasicrystals, eds. F. Axel and D. Gratias, Springer, Berlin (1995), pp. 3-16. 
[83] R. V. Moody, Meyer sets and their duals, in: The Mathematics of Long-Range Aperiodic Order, NATO ASI Series C 489, Kluwer, Dordrecht (1997), pp. 403-441.

[84] D. Osadchy, J. Avron, Hofstadter butterfly as a quantum phase diagram, J. Math. Phys., 42, 5665, (2001)

[85] K. R. Parthasarathy, Probability Measures on Metric Spaces, Academic Press, New York (1967).

[86] L. Pastur, A. Figotin, Spectra of Random and Almost-Periodic Operators, Springer, (1992).

[87] G. K. Pedersen, $C^{*}$-Algebras and their Automorphism Groups, Academic Press, London (1979).

[88] R.E. Peierls, Zur Theorie der elektrischen und thermischen Leitfähigkeit von Metallen, Ann. Phys., 4, 121-148, (1930).

[89] R.E. Peierls, Zur Theorie des Diamagnetismus von Leitungelectronen, Z. für Phys., 80, 763-791, (1933).

[90] R. Penrose, Pentaplexity, Math. Intelligencer, Bull. Inst. Math. Appl., 2, 32-37, (1979).

[91] M. Pimsner and D. Voiculescu, Exact sequences for K-groups and Ext-group of certain cross-products of $C^{*}$-algebra, J. Operator Theory, 4, 93-118, (1980).

[92] M. Pimsner and D. Voiculescu, Imbedding the irrational rotation $C^{*}$-algebra into an AF algebra, J. Operator Theory, 4, 201-211, (1980).

[93] R.E. Prange, Quantized Hall resistance and the measurement of the fine structure constant, Phys. Rev. B, 23, 4802-4805, (1981).

[94] M. Queffelec, Substitution dynamical systems-Spectral analysis, Lecture Notes in Math., Springer, Berlin Heidelberg New York 1987, vol. 1294.

[95] R. Rammal, J. Bellissard, An algebraic semiclassical approach to Bloch electrons in a magnetic field, J. Phys. France, 51, 1803-1830, (1990).

[96] J. Renault, A Groupoid Approach to $C^{*}$-Algebras, Lecture Notes in Math., 793, Springer, Berlin (1980).

[97] M.A. Rieffel, $C^{*}$-algebras associated with irrational rotations, Pac. J. Math., 95 (2), 415-419, (1981). 
[98] M.A. Rieffel, (i) Morita equivalence for operator algebras, in Operator algebras and applications, ed. R. V. Kadison, Amer. Math. Soc., Providence, RI (1982), pp. 285-298; (ii) Applications of strong Morita equivalence to transformation group $C^{*}$ algebras, ed. R. V. Kadison, Amer. Math. Soc., Providence, RI (1982), pp. 299-310.

[99] S. Roche, D. Mayou \& G. Trambly de Laissardière, Electronic transport properties of quasicrystals, J. Math. Phys., 38, 1794-1822, (1997).

[100] D. Ruelle, Statistical Mechanics, Benjamin, (1969).

[101] I. Segal, A non-commutative extension of abstract integration, Ann. of Math., 57, 401-457, (1953); Correction to "A non-commutative extension of abstract integration", Ann. of Math., 58, 595-596, (1953).

[102] D. Shechtman, I. Blech, D. Gratias, J. W. Cahn, Metallic Phase with Long-Range Orientational Order and No Translational Symmetry, Phys. Rev. Lett. 51, 1951-1953 (1984).

[103] L. Sadun, R.F. Williams, Tiling Spaces are Cantor Set Fiber Bundles, to appear in Ergodic Theory and Dynamical Systems (2002).

[104] B.I. Shklovskii \& A.L. Efros, Electronic Properties of Doped Semiconductors, Springer, (1984).

[105] D. Spehner, Contributions à la théorie du transport électronique dissipatif dans les solides apériodiques, $\mathrm{Ph}$. D. Thesis, Toulouse, (2000).

[106] M. Stone Ed., The Quantum Hall Effect, World Scientific, Singapore, (1992).

[107] M. J. O. Strutt, Wirbelströme im elliptischen Zylinder, Ann. d. Phys, 84, 485-506, (1927); Eigenschwingungen einer Saite mit sinusförmiger Massenverteilung, Ann. d. Phys, 85, 129-136, (1928).

[108] M. Takesaki, Theory of operator algebras. I., Springer-Verlag, New York-Heidelberg, (1979).

[109] D.J. Thouless, Localisation and the two-dimensional Hall effect, J. Phys. C, 14, 3475-3780, (1981).

[110] D. Thouless, M. Kohmoto, M. Nightingale, M. den Nijs, Quantized Hall conductance in two-dimensional periodic potential, Phys. Rev. Lett., 49, 405-408, (1982).

[111] D.J. Thouless, Percolation and Localization, in Ill Condensed Matter, Les Houches Session XXXI 1978, edited by R. Balian (Saclay), R. Maynard (Grenoble) \& G. Toulouse (ENS), North-Holland/World Scientific, (1984). 
[112] D.C. Tsui, H.L. Stormer, A.C. Gossard, Two-Dimensional Magnetotransport in the Extreme Quantum Limit, Phys. Rev. Lett., 48, 1559-1562, (1982).

[113] W.-M. Wang, Microlocalization, percolation, and Anderson localization for the magnetic Schrödinger operator with a random potential, J. Funct. Anal., 146, 1-26, (1997).

[114] N. E. Wegge-Olsen, K-Theory and $C^{*}$-Algebras: A Friendly Approach, Oxford University Press, Oxford (1993).

[115] E. Wigner, F. Seitz, On the constitution of metallic sodium, Phys. Rev., 43, 804-810, (1933).

[116] R. Willett, J.P. Eisenstein, H.L. Störmer, D.C. Tsui, A.C. Gossard, J.H. English, Observation of an even-denominator quantum number in the fractional quantum Hall effect, Phys. Rev. Lett.. 59, 1776-1779, (1987); see also J.P. Eisenstein, H.L. Störmer, The Fractional Quantum Hall Effect, in Science, 248, 1510-1516, (1990).

[117] S. L. Woronowicz, Unbounded elements affiliated with $C^{*}$-algebras and non-compact quantum groups, Commun. Math. Phys., 136, 399-432 (1991);

[118] S. L. Woronowicz, K. Napiórkowsky, Operator theory in the $C^{*}$-algebra framework, Rep. Math. Phys., 31, 353-371 (1992).

[119] J. Zak, Magnetic translation group, Phys. Rev., A 134, 1602-1606, (1964). 